\title{
Catalytic Selenium-Promoted Intermolecular Friedel-Crafts Alkylation with Simple Alkenes
}

\author{
E Tang,* Yinjiao Zhao, Wen Li, Weilin Wang, Meng Zhang, Xin Dai \\ Key Laboratory of Medicinal Chemistry for Natural Resources (Yunnan University) Ministry of \\ Education and School of Chemical Science and Technology, Yunnan University, 2 Green Lake North \\ Road, Kunming 650091, China
}

\section{Supporting Information}

Table of Contents

General Information.

Experimental procedures of products $\mathbf{3}$...

Experimental procedures of product 5ab, 5ha, and 5ia...

Experimental procedures of products $\mathbf{7 b}, \mathbf{7 c}$, and $\mathbf{8 c}$

Experimental procedures of compounds 3ak and 3ak', 3ck and 3ck' $. \mathrm{S} 3-\mathrm{S} 4$

Characterization of the products $3,5 \mathbf{a b}, 5 \mathbf{h a}, 5 \mathbf{i a}, 7 \mathbf{b}, 7 \mathbf{c}, 8 \mathbf{c}, 3 \mathbf{a k}$ and $3 \mathbf{a k}^{\prime}$, 3ck and $3 \mathbf{c k}{ }^{\prime}$. S4-S14

The data summarized from HSQC and HMBC Spectra for compound 3ae. S14-S15

The structure of compound 3ae deduced from the ${ }^{1} \mathrm{H}^{-1} \mathrm{H}$ COSY, HSQC, HMBC, and ROESY Spectra.. .S15-S16

NOE correlation and relative configuration analysis of products $( \pm)-3$ ha and $( \pm)-3 i a .$. S16-S17

Byproducts of intermolecular selenium-promoted $\mathrm{F}-\mathrm{C}$ alkylation with styrene. .S17-S19

Characterization of compounds $18 f, 18 j, 18 i$, and $18 \mathrm{l}$. ..S19-S20

ReactIR $^{\mathrm{TM}}$ in situ FTIR analysis of the $\mathrm{F}-\mathrm{C}$ alkylation reaction. S20-S21

$\mathrm{X}$-ray structure of compound $\mathbf{3 j a}$. S21-S29

$\mathrm{X}$-ray structure of compound $\mathbf{5 a b}$. S29-S35

References. S35

Copies of NMR Spectra for compounds. S36-S73 


\section{General Information}

All reactions were performed in oven-dried glassware under an atmosphere of dry nitrogen $\left(\mathrm{N}_{2}\right)$, unless otherwise noted. Commercial grade reagents were purchased from Aladdin and Acros and used without further purification. All solvents were dried and distilled before use by standard procedures. $N$-Phenylselenophthalimide (NPSP) ${ }^{[1]}$ and Chiral diselenide $\mathbf{9}^{[2]}$ were prepared according to the literatures. The ${ }^{1} \mathrm{H}$ NMR and ${ }^{13} \mathrm{C}$ NMR spectra were recorded on Bruker Advance-400 or Bruker Advance-300. ${ }^{1} \mathrm{H}$ NMR splitting patterns are designated as singlet (s), doublet (d), triplet (t), or quartet (q). Splitting patterns that could not be interpreted or easily visualized are designated as multiplet (m) or broad (br). COSY, ROSY, HMBC and HSQC are used to determine the regioselectivity of products. High resolution mass spectra (HRMS) were obtained by the ESI method from Agilent LC/MSD TOF instrument. The infrared (IR) spectra were acquired on a FT-IR spectrometer. ReactIR ${ }^{\mathrm{TM}}$ spectra were acquired on a ReactIR 15 in situ mid-infrared based system with a diamond tiber-optics probe $(9.5 \mathrm{~mm})$ and an $\mathrm{iC} \mathrm{IR} 4.3$ control and analysis software.

\section{Optimization of intermolecular selenium-promoted $\mathrm{F}-\mathrm{C}$ alkylation with styrene}

Table 1. Optimization of Intermolecular Selenium-Promoted F-C Alkylation of 1,3,5-Trimethoxybenzene with Styrene and NPSP ${ }^{a}$

\begin{tabular}{|c|c|c|c|}
\hline Entry & Jawic onid & Temn $\left({ }^{\circ} \mathrm{C}\right)$ & Yield of $3 \mathrm{ag}^{b}(\%)$ \\
\hline & & & \\
\hline 1 & none & $\mathrm{rt}$ & $\mathrm{nr}$ \\
\hline 2 & $\mathrm{TiCl}_{4}(0.1$ equiv $)$ & $\mathrm{rt}$ & $\mathrm{nr}$ \\
\hline 3 & $\mathrm{AlCl}_{3}(0.1$ equiv $)$ & $\mathrm{rt}$ & $\mathrm{nr}$ \\
\hline 4 & $\mathrm{FeCl}_{3}(0.1$ equiv $)$ & $\mathrm{rt}$ & $\mathrm{nr}$ \\
\hline 5 & $\mathrm{ZnCl}_{2}$ (0.1 equiv) & $\mathrm{rt}$ & $\mathrm{nr}$ \\
\hline 6 & $\mathrm{SnCl}_{4}(0.1$ equiv $)$ & $\mathrm{rt}$ & $\mathrm{nr}$ \\
\hline 7 & $\mathrm{BF}_{3} \cdot \mathrm{Et}_{2} \mathrm{O}$ (0.1 equiv) & $\mathrm{rt}$ & $\mathrm{nr}$ \\
\hline 8 & $\operatorname{AgOTf}(0.1$ equiv) & -78 to $\mathrm{rt}$ & $\mathrm{nr}$ \\
\hline 9 & $\operatorname{Sm}(\mathrm{OTf})_{3}(0.1$ equiv $)$ & -78 to $\mathrm{rt}$ & $\mathrm{nr}$ \\
\hline 10 & TMSOTf ( 0.1 equiv) & $\mathrm{rt}$ & $\mathrm{nr}$ \\
\hline 11 & TMSOTf ( 0.1 equiv) & 0 & $\mathrm{nr}$ \\
\hline 12 & TMSOTf ( 0.1 equiv) & -78 & 15 \\
\hline 13 & TMSOTf ( 0.1 equiv) & -78 to $-20^{\mathrm{c}}$ & 97 \\
\hline 14 & TMSOTf ( 0.05 equiv) & -78 to $-20^{c}$ & 54 \\
\hline 15 & TMSOTf ( 0.2 equiv) & -78 to $-20^{\mathrm{c}}$ & 95 \\
\hline 16 & TMSOTf ( 0.4 equiv) & -78 to $-20^{\mathrm{c}}$ & 92 \\
\hline
\end{tabular}


${ }^{a}$ The reaction was performed on a $1.0 \mathrm{mmol}$ scale in $10 \mathrm{~mL}$ of $\mathrm{CH}_{2} \mathrm{Cl}_{2}$ for $9 \mathrm{~h}$ with a $\mathbf{1 a} / \mathbf{2 a}$ /NPSP molar ratio of 1/1/1.10. ${ }^{b}$ Isolated yield of $\mathbf{3 a a}$ after column chromatography.

${ }^{c}$ The reaction was performed at $-78{ }^{\circ} \mathrm{C}$ for $2 \mathrm{~h}$ and then at $-20{ }^{\circ} \mathrm{C}$ for $7 \mathrm{~h}$.

\section{Experimental procedures of products 3}

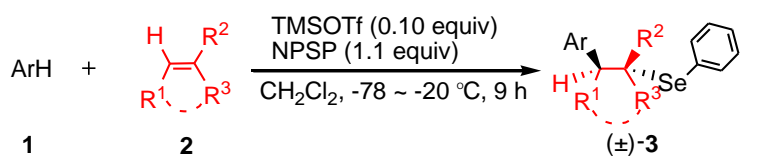

To a stirred solution of NPSP $(166.2 \mathrm{mg}, 0.55 \mathrm{mmol})$ in $\mathrm{CH}_{2} \mathrm{Cl}_{2}(5 \mathrm{~mL})$ at $-78{ }^{\circ} \mathrm{C}$, TMSOTf $(9.05 \mu \mathrm{L}, 0.05$ $\mathrm{mmol})$ was added. The reaction mixture was stirred for $30 \mathrm{~min}$ and the alkenes $(0.5 \mathrm{mmol})$ were added. Then the reaction mixture was stirred for $30 \mathrm{~min}$ and arenes $(0.5 \mathrm{mmol})$ were added. The reaction mixture was stirred for another $2 \mathrm{~h}$ at $-78{ }^{\circ} \mathrm{C}$ and then allowed to slowly warm to $-20{ }^{\circ} \mathrm{C}$ (about for $7 \mathrm{~h}$ ). After the completion of the reaction, the reaction was quenched with saturated aqueous $\mathrm{NaHCO}_{3}$ solution. The mixture was extracted with $\mathrm{CH}_{2} \mathrm{Cl}_{2}(3 \times 10 \mathrm{~mL})$. The organic layer was dried over anhydrous $\mathrm{MgSO}_{4}$ and filtered. After removal of the solvents under reduced pressure, the crude products were purified by flash chromatography on silica Gel (elution with petroleum ether/ethyl acetate: 10/1) to give the products 3.

Experimental procedures of product 5ab, 5ha, and 5ia

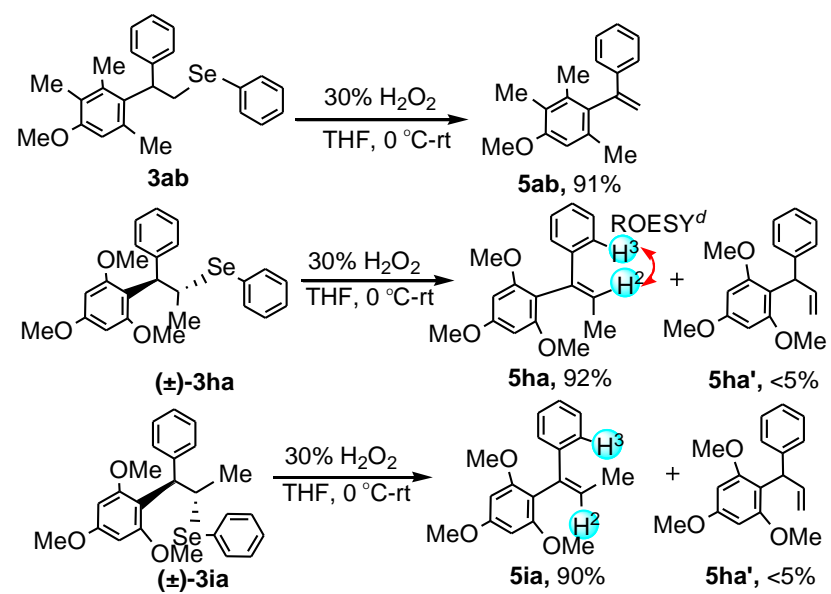

$30 \%$ Aqueous $\mathrm{H}_{2} \mathrm{O}_{2}(0.5 \mathrm{~mL})$ solution was added to a solution of 3ab or 3ha or 3ia $(0.2 \mathrm{mmol})$ in THF $(8 \mathrm{~mL})$ at $0{ }^{\circ} \mathrm{C}$. The reaction mixture was stirred at $0{ }^{\circ} \mathrm{C}$ for $1.5 \mathrm{~h}$ and then stirred at room temperature for 30 minutes to $3 \mathrm{~h}$. After the completion of the reaction, it was quenched with saturated aqueous $\mathrm{NaHSO}_{3}$ solution and the reaction mixture was extracted with $\mathrm{CH}_{2} \mathrm{Cl}_{2}(3 \times 10 \mathrm{~mL})$. The organic phase was dried over anhydrous $\mathrm{MgSO}_{4}$, filtered, and concentrated under reduced pressure. The residue was purified by flash chromatography on silica Gel (elution with petroleum ether/ethyl acetate: 10/1) to give $\mathbf{5 a b}$ (46 $\mathrm{mg}, 91 \%)$ or $\mathbf{5 h a}$ (52 $\mathrm{mg}, 92 \%)$ or 5 ia $(51 \mathrm{mg}, 90 \%)$. 


\section{Experimental procedures of products $7 \mathrm{~b}, 7 \mathrm{c}, 5 \mathrm{ab}$ and $8 \mathrm{c}$}

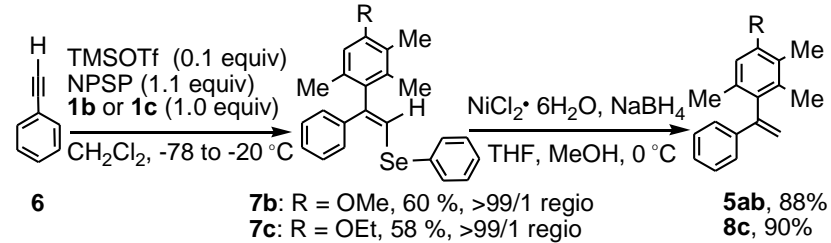

To a stirred solution of NPSP $(166.2 \mathrm{mg}, 0.55 \mathrm{mmol})$ in $\mathrm{CH}_{2} \mathrm{Cl}_{2}(5 \mathrm{~mL})$ at $-78{ }^{\circ} \mathrm{C}$ was added TMSOTf $(9.05$ $\mu \mathrm{L}, 0.05 \mathrm{mmol})$. The reaction mixture was allowed to stir at $-78{ }^{\circ} \mathrm{C}$ for $30 \mathrm{~min}$. Phenylacetylene $6(51 \mathrm{mg}$, $0.5 \mathrm{mmol}$ ) was added to the vessel and the resulting mixture was allowed to stir at $-78{ }^{\circ} \mathrm{C}$ for another $30 \mathrm{~min}$ and then treated with 1-methoxy-2,3,5-trimethylbenzene (1b, $75 \quad \mathrm{mg}, 0.5 \mathrm{mmol})$ or 1-ethoxy-2,3,5-trimethylbenzene $(\mathbf{1 c}, 82 \mathrm{mg}, 0.5 \mathrm{mmol})$ via springe. The yellow mixture was aged at $-78{ }^{\circ} \mathrm{C}$ for $2 \mathrm{~h}$ and then at $-20^{\circ} \mathrm{C}$ for another $7 \mathrm{~h}$. After quenching with saturated aqueous solution of $\mathrm{NaHCO}_{3}$, the mixtrure was sequentially extracted with $\mathrm{CH}_{2} \mathrm{Cl}_{2}(3 \times 10 \mathrm{~mL})$, dried over anhydrous $\mathrm{MgSO}_{4}$, filtered, and concentrated in vacuo to afford the yellow crude product. Purification by flash column chromatography on silica Gel using petroleum ether and ethyl acetate (10/1) as an eluent afforded the desired $\beta$-(phenylseleno) styrene $7 \mathbf{b}(122.2 \mathrm{mg}, 60 \%)$ or $7 \mathbf{c}(122.2 \mathrm{mg}, 58 \%)$ as yellow viscous liquid.

To a stired solution of Nickel chloride hexahydrate $(109 \mathrm{mg}, 0.46 \mathrm{mmol})$ in methanol $(2 \mathrm{~mL})$ was added a solution of $\beta$-(phenylseleno) styrene $7 \mathbf{b}(82 \mathrm{mg}, 0.2 \mathrm{mmol})$ or $7 \mathbf{c}(84 \mathrm{mg}, 0.2 \mathrm{mmol})$ in THF $(2 \mathrm{~mL})$ via springe. The reaction mixture was cooled to $0{ }^{\circ} \mathrm{C}$ (ice bath) and treated with sodium borohydride $\left(\mathrm{NaBH}_{4}\right.$, $51.4 \mathrm{mg}, 1.36 \mathrm{mmol})$ in small portions. During which time an immediate vigorous reaction occurred with evolution of hydrogen gas and the formation of a black precipitate. After the addition of $\mathrm{NaBH}_{4}$ was completed, the reaction mixture was continued to stir for an additional $15 \mathrm{~min}$, filtered, and washed with methanol and THF. The filtrate was evaporated in vacuo and the residue was purified by flash column chromatography on silica Gel (elution with petroleum ether/ethyl acetate: 10/1) to give $\mathbf{5 a b}$ (44 $\mathrm{mg}, 88 \%$ ) or 8c (48 mg, 90\%) as light yellow solid.

\section{Experimental procedures of compounds 3ak and 3ak', 3ck and 3ck'}

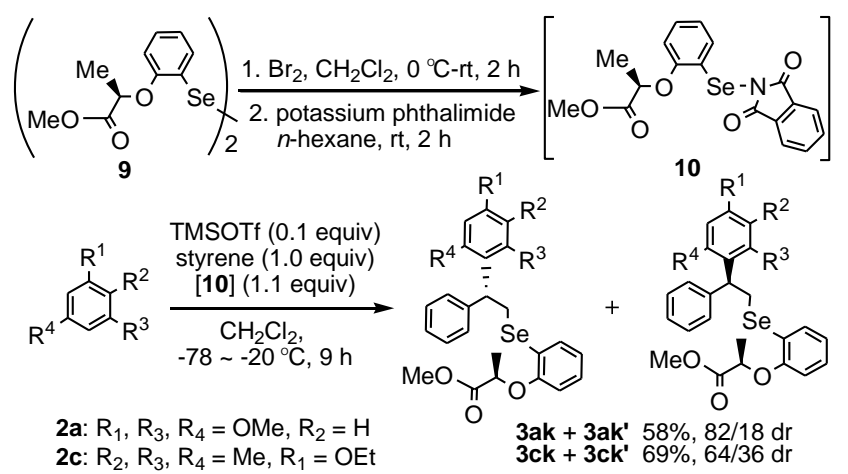

$\left(2 R, 2^{\prime} R\right)$-Dimethyl-2,2'-[diselenobis(2,1-phenyleneoxy)]bispropionate $\left(9^{[2]}, 284 \mathrm{mg}, \quad 0.55 \mathrm{mmol}\right)$ was dissolved in anhydrous $\mathrm{CH}_{2} \mathrm{Cl}_{2}(5 \mathrm{~mL})$, and the solution was cooled to $0{ }^{\circ} \mathrm{C}$, bromine $(31 \mu \mathrm{L}, 0.6 \mathrm{mmol})$ was added dropwise to the stirring solution. The mixture was stirred for $0.5 \mathrm{~h}$, allowed to warm to room temperature, and stirred for an additional $2 \mathrm{~h}$. The solution was concentrated under reduced pressure to provide (2R)-methyl-2-[bromoseleno-(2,1-phenyleneoxy)]propionate as a rust liquid which was used in the next step without any treatment.

(2R)-Methyl-2-[bromoseleno-(2,1-phenyleneoxy)]propionate, anhydrous hexane $(5 \mathrm{~mL})$, and phthalimide potassium salt (204 mg, $1.1 \mathrm{mmol}$ ) were added to a round-bottom flask under an atmosphere of dry nitrogen. 
The resulting mixture was stirred rapidly at room temperature for $2 \mathrm{~h}$, and finally the solution turned yellow. To this solution was added anhydrous $\mathrm{CH}_{2} \mathrm{Cl}_{2}(15 \mathrm{~mL})$, and then the reaction mixture was filtered. The filtrate was concentrated under reduced pressure and then anhydrous $\mathrm{CH}_{2} \mathrm{Cl}_{2}(5 \mathrm{~mL})$ was added. The mixture was stirred and cooled to $-78{ }^{\circ} \mathrm{C}$ and TMSOTf $(18 \mu \mathrm{L}, 0.1 \mathrm{mmol})$ was added at this temperature. The reaction mixture was stirred for $30 \mathrm{~min}$ and the alkenes $(1.0 \mathrm{mmol})$ were added. Then the solution was stirred for $30 \mathrm{~min}$ and arenes $\mathbf{2 a}$ or $\mathbf{2 c}(1.0 \mathrm{mmol})$ were added and the reaction mixture was stirred for another $2 \mathrm{~h}$ at $-78{ }^{\circ} \mathrm{C}$. Then the reaction mixture was allowed to slowly warm to $-20{ }^{\circ} \mathrm{C}$ (for $7 \mathrm{~h}$ ) and quenched with saturated aqueous $\mathrm{NaHCO}_{3}$ solution. The mixture was extracted with $\mathrm{CH}_{2} \mathrm{Cl}_{2}(3 \times 10 \mathrm{~mL})$ and the organic layer was dried over anhydrous $\mathrm{MgSO}_{4}$ and filtered. After removal of the solvents under reduced pressure, the crude products were purified by flash chromatography on silica Gel (elution with petroleum ether/ethyl acetate: 10/1) to give the products 3ak and 3ak' (307 mg, 58\% combined yield) or 3ck and 3ck' (363 mg, 69\% combined yield).

\title{
Characterization of compounds 3, 5ab, 5ha, 5ia, 7b, 7c, 8c, 9, 3ak and 3ak', 3ck and 3ck'
}

\section{2,4,6-Trimethoxy-1-[1-phenyl-2-(phenylseleno)ethyl]benzene (3aa)}

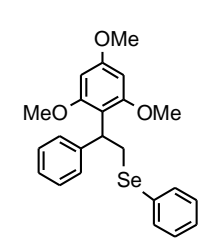

\author{
Yellow viscous liquid.
}

Yield: $208 \mathrm{mg}$, 97\%.

${ }^{1}$ H NMR (300 MHz, $\left.\mathrm{CDCl}_{3}\right): \delta 7.43-7.40(\mathrm{~m}, 2 \mathrm{H}), 7.31(\mathrm{~d}, J=7.2 \mathrm{~Hz}, 2 \mathrm{H}), 7.21-7.06(\mathrm{~m}$, $6 \mathrm{H}), 6.05(\mathrm{~s}, 2 \mathrm{H}), 4.94(\mathrm{dd}, J=7.2,9.6 \mathrm{~Hz}, 1 \mathrm{H}), 3.89(\mathrm{dd}, J=9.6,11.4 \mathrm{~Hz}, 1 \mathrm{H}), 3.75-3.68$ $(\mathrm{m}, 4 \mathrm{H}), 3.61(\mathrm{~s}, 6 \mathrm{H})$.

${ }^{13}$ C NMR (75 MHz, $\left.\mathrm{CDCl}_{3}\right): \delta 159.9,159.1,144.2,132.5,131.4,128.7,127.8,127.8$, $126.3,125.7,112.3,91.1,55.5,55.1,40.2,31.5$.

IR $\left(\mathrm{KBr}, \mathrm{cm}^{-1}\right): v$ 3052, 3003, 2942, 2837, 1595, 1462, 1329, 1205, 1127, 1048, 949, 899, $813,739,694$.

HRMS $\left(\mathrm{ESI}^{+}\right)$: calcd for $\left[\mathrm{C}_{23} \mathrm{H}_{24} \mathrm{NaO}_{3} \mathrm{Se}^{+}:\right.$: 451.0788, found 451.0781 .

\section{4-Methoxy-2,3,6-trimethyl-1-[1-phenyl-2-(phenylseleno)ethyl]benzene (3ab)}



Yellow viscous liquid.

Yield: $162 \mathrm{mg}, 79 \%$.

${ }^{1}$ H NMR $\left(300 \mathrm{MHz}, \mathrm{CDCl}_{3}\right): \delta$ 7.44-7.41 (m, 2H), 7.25-7.10 (m, 8H), $6.51(\mathrm{~s}, 1 \mathrm{H}), 4.87(\mathrm{t}$, $J=7.5 \mathrm{~Hz}, 1 \mathrm{H}), 3.88(\mathrm{dd}, J=7.2,12 \mathrm{~Hz}, 1 \mathrm{H}), 3.75$ (s, 3H), 3.59 (dd, $J=8.4,11.7 \mathrm{~Hz}, 1 \mathrm{H})$, $2.20-1.90$ (br, 9H).

${ }^{13}$ C NMR $\left(75 \mathrm{MHz}, \mathrm{CDCl}_{3}\right): \delta 155.9,143.8,137.0,135.1,132.9,132.6,131.1,128.9$, $128.3,127.0,126.9,125.7,110.7,55.3,44.4,31.5,22.0,17.4,12.0$.

IR $\left(\mathrm{KBr}, \mathrm{cm}^{-1}\right): v 3060,2831,1583,1479,1448,1385,1298,1196,1117,1070,1016,905$, $838,743,699$.

HRMS (ESI ${ }^{+}$): calcd for $\left[\mathrm{C}_{24} \mathrm{H}_{27} \mathrm{OSe}\right]^{+}: 411.1227$, found 411.1223 . 
4-Ethoxy-2,3,6-trimethyl-1-[1-phenyl-2-(phenylseleno)ethyl]benzene (3ac)

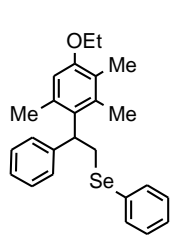

Yellow viscous liquid.

Yield: $172 \mathrm{mg}, 81 \%$.

${ }^{1}$ H NMR (300 MHz, $\left.\mathrm{CDCl}_{3}\right): \delta$ 7.43-7.40 (m, 2H), 7.23-7.08 (m, 8H), $6.50(\mathrm{~s}, 1 \mathrm{H}), 4.85$ (t, $J=7.5 \mathrm{~Hz}, 1 \mathrm{H}), 3.97-3.84(\mathrm{~m}, 3 \mathrm{H}), 3.58(\mathrm{dd}, J=8.4,11.7 \mathrm{~Hz}, 1 \mathrm{H}), 2.16-1.90$ (br, 9H), $1.36(\mathrm{t}, J=6.9 \mathrm{~Hz}, 3 \mathrm{H})$.

${ }^{13}$ C NMR $\left(75 \mathrm{MHz}, \mathrm{CDCl}_{3}\right): \delta 155.4,143.8,136.9,134.9,132.9,132.6,131.1,128.9$, 128.2, 127.0, 126.8, 125.7, 123.9, 111.8, 63.5, 44.5, 31.5, 21.9, 17.4, 15.2, 12.1 .

IR $\left(\mathrm{KBr}, \mathrm{cm}^{-1}\right): v$ 3048, 2970, 2928, 2872, 1584, 1476, 1387, 1291, 1212, 1107, 1025, 905 , $838,737,690$.

HRMS $\left(\mathrm{ESI}^{+}\right)$: calcd for $\left[\mathrm{C}_{25} \mathrm{H}_{29} \mathrm{OSe}\right]^{+}: 425.1384$, found 425.1388 .

\section{4-Ethoxy-5-isopropyl-2-methyl-1-[1-phenyl-2-(phenylseleno)ethyl]benzene (3ad)}

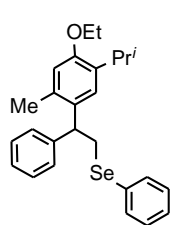

Yellow viscous liquid.

Yield: $164 \mathrm{mg}, 75 \%$.

${ }^{1}$ H NMR (300 MHz, $\left.\mathrm{CDCl}_{3}\right): \delta 7.45-7.42(\mathrm{~m}, 2 \mathrm{H}), 7.22-7.10(\mathrm{~m}, 9 \mathrm{H}), 6.56(\mathrm{~s}, 1 \mathrm{H}), 4.38$ $(\mathrm{t}, J=7.8 \mathrm{~Hz}, 1 \mathrm{H}), 3.95(\mathrm{q}, J=6.9 \mathrm{~Hz}, 2 \mathrm{H}), 3.61-3.47(\mathrm{~m}, 2 \mathrm{H}), 3.35-3.26(\mathrm{~m}, 1 \mathrm{H}), 2.10(\mathrm{~s}$, $3 \mathrm{H}), 1.36(\mathrm{t}, J=6.9 \mathrm{~Hz}, 3 \mathrm{H}), 1.23(\mathrm{~d}, J=7.2 \mathrm{~Hz}, 3 \mathrm{H}), 1.20(\mathrm{~d}, J=7.8 \mathrm{~Hz}, 3 \mathrm{H})$.

${ }^{13}$ C NMR $\left(75 \mathrm{MHz}, \mathrm{CDCl}_{3}\right): \delta 154.7,143.9,134.4,132.9,132.8,131.2,129.1,128.5$, $128.1,126.9,126.4,124.5,113.8,63.5,47.1,34.5,27.1,22.9,19.7,15.1$.

IR $\left(\mathrm{KBr}, \mathrm{cm}^{-1}\right): v 3055,2963,2868,1579,1493,1446,1393,1345,1264,1189,1103$, 1058, 900, 840, 736, 694 .

HRMS $\left(\mathrm{ESI}^{+}\right)$: calcd for $\left[\mathrm{C}_{26} \mathrm{H}_{31} \mathrm{OSe}\right]^{+}:$439.1540, found 439.1543 .

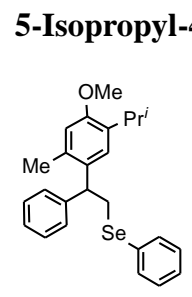

-methoxy-2-methyl-1-[1-phenyl-2-(phenylseleno)ethyl]benzene (3ae)

Yellow viscous liquid.

Yield: $157 \mathrm{mg}, 74 \%$.

${ }^{1} \mathbf{H}$ NMR $\left(300 \mathrm{MHz}, \mathrm{CDCl}_{3}\right): \delta$ 7.45-7.42 (m, 2H), 7.24-7.09 (m, 9H), 6.57 (s, 1H), 4.39 (t, $J=7.8 \mathrm{~Hz}, 1 \mathrm{H}), 3.71(\mathrm{~s}, 3 \mathrm{H}), 3.61-3.47(\mathrm{~m}, 2 \mathrm{H}), 3.36-3.23(\mathrm{~m}, 1 \mathrm{H}), 2.12(\mathrm{~s}, 3 \mathrm{H}), 1.23$ $(\mathrm{d}, J=6.9 \mathrm{~Hz}, 3 \mathrm{H}), 1.20(\mathrm{~d}, J=6.9 \mathrm{~Hz}, 3 \mathrm{H})$.

${ }^{13} \mathrm{C}$ NMR $\left(75 \mathrm{MHz}, \mathrm{CDCl}_{3}\right.$ ): $\delta$ 155.1, 143.8, 134.4, 134.2, 132.8, 132.8, 131.1, 129.0, 128.5, 128.1, 126.8, 126.4, 124.4, 112.8, 55.3, 47.1, 34.4, 26.8, 22.9, 19.7 .

IR $\left(\mathrm{KBr}, \mathrm{cm}^{-1}\right):$ v 3060, 3023, 2954, 1580, 1495, 1451, 1346, 1265, 1190, 1062, 897, 843, 737,694 .

HRMS $\left(\mathrm{ESI}^{+}\right)$calcd for $\left[\mathrm{C}_{25} \mathrm{H}_{29} \mathrm{OSe}\right]^{+}:$425.1384, found 425.1380 .

\section{2,4-Dimethoxy-1-[1-phenyl-2-(phenylseleno)ethyl]benzene (3af)}<smiles>COc1ccc(C2c3ccccc3O[Hg]2c2ccccc2)c(OC)c1</smiles>
Yellow viscous liquid.

Yield: $129 \mathrm{mg}, 65 \%$.

${ }^{1}$ H NMR $\left(300 \mathrm{MHz}, \mathrm{CDCl}_{3}\right): \delta 7.46-7.44(\mathrm{~m}, 2 \mathrm{H}), 7.23-7.14(\mathrm{~m}, 8 \mathrm{H}), 7.01(\mathrm{~d}, J=8.7 \mathrm{~Hz}$, $1 \mathrm{H}), 6.39(\mathrm{~d}, J=1.2 \mathrm{~Hz}, 2 \mathrm{H}), 4.65(\mathrm{t}, J=7.5 \mathrm{~Hz}, 1 \mathrm{H}), 3.71(\mathrm{~s}, 3 \mathrm{H}), 3.67(\mathrm{~s}, 3 \mathrm{H}), 3.62-3.43$ $(\mathrm{m}, 2 \mathrm{H})$.

${ }^{13}$ C NMR (75 MHz, $\left.\mathrm{CDCl}_{3}\right): \delta 159.6,157.9,143.6,132.5,131.3,128.9,128.5,128.3$, 128.2, 126.6, 126.4, 124.6, 104.0, 98.8, 55.4, 55.3, 44.1, 32.8 . 
IR $\left(\mathrm{KBr}, \mathrm{cm}^{-1}\right): v$ 3056, 2999, 2934, 2841, 1599, 1498, 1457, 1293, 1202, 1150, 1035, 926, $831,736,695$.

HRMS $\left(\mathrm{ESI}^{+}\right)$: calcd for $\left[\mathrm{C}_{22} \mathrm{H}_{22} \mathrm{NaO}_{2} \mathrm{Se}\right]^{+}:$421.0683, found 421.0680.

\section{2-Ethoxy-4-methoxy-1-[1-phenyl-2-(phenylseleno)ethyl]benzene (3ag) and}

2-Methoxy-4-ethoxy-1-[1-phenyl-2-(phenylseleno)ethyl]benzene (3ag')

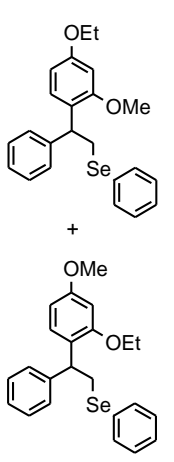

Yellow viscous liquid.

Yield: $142 \mathrm{mg}, 69 \%$.

${ }^{1}$ H NMR $\left(300 \mathrm{MHz}, \mathrm{CDCl}_{3}\right): \delta 7.47-7.44(\mathrm{~m}, 2 \mathrm{H}), 7.24-7.14(\mathrm{~m}, 8 \mathrm{H}), 7.01(\mathrm{dd}, J=8.1$, $11.7 \mathrm{~Hz}, 1 \mathrm{H}), 6.40-6.36(\mathrm{~m}, 2 \mathrm{H}), 4.64(\mathrm{t}, J=8.1 \mathrm{~Hz}, 1 \mathrm{H}), 3.98-3.82(\mathrm{~m}, 2 \mathrm{H}), 3.70(\mathrm{~m}$, $1.3 \mathrm{H}), 3.70(\mathrm{~m}, 1.7 \mathrm{H}), 3.64-3.43(\mathrm{~m}, 2 \mathrm{H}), 1.37-1.26(\mathrm{~m}, 3 \mathrm{H})$.

${ }^{13}$ C NMR $\left(75 \mathrm{MHz}, \mathrm{CDCl}_{3}\right): \delta 159.0,157.3,143.6,132.5,131.3,128.9,128.5,128.4$, $128.3,126.8,126.4,124.6,104.7,99.3,63.5,55.4,44.3,32.9,14.9$.

IR $\left(\mathrm{KBr}, \mathrm{cm}^{-1}\right): v$ 3048, 2975, 2921, 1593, 1492, 1442, 1271, 1189, 1119, 1041, 969, 898, $829,735,694$.

HRMS $\left(\mathrm{ESI}^{+}\right)$: calcd for $\left[\mathrm{C}_{23} \mathrm{H}_{25} \mathrm{O}_{2} \mathrm{Se}\right]^{+}: 413.1020$, found 413.1023

\section{2,4,6-Trimethoxy-1-[1-(4-fluorophenyl)-2-(phenylseleno)ethyl]benzene (3ba)}

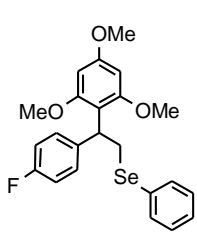

Yellow viscous liquid.

Yield: $176 \mathrm{mg}, 79 \%$.

${ }^{1} \mathbf{H}$ NMR $\left(300 \mathrm{MHz}, \mathrm{CDCl}_{3}\right): \delta 7.43-7.40(\mathrm{~m}, 2 \mathrm{H}), 7.30-7.14(\mathrm{~m}, 5 \mathrm{H}), 6.88(\mathrm{t}, J=9 \mathrm{~Hz}$, 2H), $6.07(\mathrm{~s}, 2 \mathrm{H}), 4.89(\mathrm{t}, J=8.4 \mathrm{~Hz}, 1 \mathrm{H}), 3.82(\mathrm{dd}, J=9,11.4 \mathrm{~Hz}, 1 \mathrm{H}), 3.72-3.65(\mathrm{~m}$, $10 \mathrm{H})$.

${ }^{13}$ C NMR $\left(75 \mathrm{MHz}, \mathrm{CDCl}_{3}\right): \delta 161.5\left(\mathrm{~d}, J_{\mathrm{CF}}=243 \mathrm{~Hz}\right), 160.0,159.0,139.9,132.6,131.2$, $129.4\left(\mathrm{~d}, J_{\mathrm{CF}}=7.5 \mathrm{~Hz}\right), 128.8,126.4,114.4\left(\mathrm{~d}, J_{\mathrm{CF}}=21 \mathrm{~Hz}\right), 112.2,91.1,55.6,55.1,39.6$, 31.5 .

IR $\left(\mathrm{KBr}, \mathrm{cm}^{-1}\right): v$ 3052, 2999, 2946, 2831, 1593, 1501, 1461, 1417, 1332, 1212, 1145, $1111,1058,948,815,735,690$.

HRMS (ESI ${ }^{+}$): calcd for $\left[\mathrm{C}_{23} \mathrm{H}_{24} \mathrm{FO}_{3} \mathrm{Se}\right]^{+}:$447.0875, found 447.0868 .

\section{4-Methoxy-2,3,6-trimethyl-1-[1-(4-fluorophenyl)-2-(phenylseleno)ethyl]benzene (3bb)}

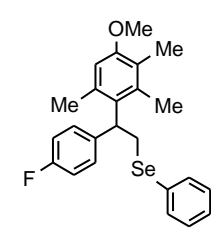

Yellow viscous liquid.

Yield: $186 \mathrm{mg}, 87 \%$.

${ }^{1}$ H NMR (300 MHz, $\left.\mathrm{CDCl}_{3}\right): \delta 7.45-7.42(\mathrm{~m}, 2 \mathrm{H}), 7.20-7.10(\mathrm{~m}, 5 \mathrm{H}), 6.92(\mathrm{t}, J=8.7 \mathrm{~Hz}$, $2 \mathrm{H}), 6.52(\mathrm{~s}, 1 \mathrm{H}), 4.80(\mathrm{t}, J=7.5 \mathrm{~Hz}, 1 \mathrm{H}), 3.82(\mathrm{dd}, J=7.5,11.7 \mathrm{~Hz}, 1 \mathrm{H}), 3.78(\mathrm{~s}, 3 \mathrm{H})$, $3.57(\mathrm{dd}, J=8.1,11.7 \mathrm{~Hz}, 1 \mathrm{H}), 2.17-1.90(\mathrm{br}, 9 \mathrm{H})$.

${ }^{13}$ C NMR $\left(75 \mathrm{MHz}, \mathrm{CDCl}_{3}\right): \delta 161.1\left(\mathrm{~d}, J_{\mathrm{CF}}=243 \mathrm{~Hz}\right), 156.0,139.4,136.9,135.0,133.1$, $132.5,130.9,129.1,128.6\left(\mathrm{~d}, J_{\mathrm{CF}}=6.8 \mathrm{~Hz}\right), 127.1,115.0\left(\mathrm{~d}, J_{\mathrm{CF}}=21 \mathrm{~Hz}\right), 110.7,55.4$, 43.9, 31.7, 22.0, 17.4, 12.0 .

IR $\left(\mathrm{KBr}, \mathrm{cm}^{-1}\right): v$ 3052, 2940, 1587, 1504, 1467, 1302, 1225, 1118, 1013, 828, 736, 682.

HRMS $\left(\mathrm{ESI}^{+}\right)$: calcd for $\left[\mathrm{C}_{24} \mathrm{H}_{26} \mathrm{FOSe}\right]^{+}: 429.1133$, found 429.1130 . 
5-Isopropyl-4-methoxy-2-methyl-1-[1-(4-fluorophenyl)-2-(phenylseleno)ethyl]benzene (3be)

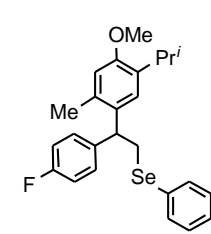

Yellow viscous liquid.

Yield: $166 \mathrm{mg}, 75 \%$.

${ }^{1} \mathbf{H}$ NMR $\left(300 \mathrm{MHz}, \mathrm{CDCl}_{3}\right): \delta 7.46-7.43(\mathrm{~m}, 2 \mathrm{H}), 7.22-7.10(\mathrm{~m}, 6 \mathrm{H}), 6.93(\mathrm{t}, J=8.7 \mathrm{~Hz}$, $2 \mathrm{H}), 6.59(\mathrm{~s}, 1 \mathrm{H}), 4.35(\mathrm{t}, J=7.8 \mathrm{~Hz}, 1 \mathrm{H}), 3.76(\mathrm{~s}, 3 \mathrm{H}), 3.58-3.42(\mathrm{~m}, 2 \mathrm{H}), 3.32-3.23(\mathrm{~m}$, $1 \mathrm{H}), 2.11(\mathrm{~s}, 3 \mathrm{H}), 1.22(\mathrm{~d}, J=7.5 \mathrm{~Hz}, 3 \mathrm{H}), 1.19(\mathrm{~d}, J=7.2 \mathrm{~Hz}, 3 \mathrm{H})$.

${ }^{13}$ C NMR $\left(75 \mathrm{MHz}, \mathrm{CDCl}_{3}\right): \delta 161.52\left(\mathrm{~d}, J_{\mathrm{CF}}=243 \mathrm{~Hz}\right), 155.3,139.5,134.5,134.4,133.0$, $132.9,130.9,129.6\left(\mathrm{~d}, J_{\mathrm{CF}}=7.5 \mathrm{~Hz}\right), 129.2,127.0,124.4,115.2\left(\mathrm{~d}, J_{\mathrm{CF}}=21 \mathrm{~Hz}\right), 113.0$, $55.4,46.4,34.6,26.9,22.9,19.7$.

IR $\left(\mathrm{KBr}, \mathrm{cm}^{-1}\right): v$ 3052, 2951, 1597, 1495, 1454, 1349, 1274, 1202, 1062, 1015, 896, 835, $741,686$.

HRMS $\left(\mathrm{ESI}^{+}\right)$: calcd for $\left[\mathrm{C}_{25} \mathrm{H}_{28} \mathrm{FOSe}\right]^{+}: 443.1289$, found 443.1280 .

\section{2,4-Dimethoxy-1-[1-(4-fluorophenyl)-2-(phenylseleno)ethyl]benzene (3bf)}

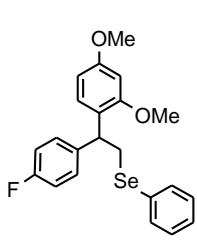

Yellow viscous liquid.

Yield: $152 \mathrm{mg}, 73 \%$.

${ }^{1} \mathbf{H}$ NMR (300 MHz, $\left.\mathrm{CDCl}_{3}\right): \delta$ 7.46-7.43 (m, 2H), 7.23-7.16 (m, 5H), 7.00-6.90 (m, 3H), $6.43-6.40(\mathrm{~m}, 2 \mathrm{H}), 4.60(\mathrm{t}, J=8.1 \mathrm{~Hz}, 1 \mathrm{H}), 3.74(\mathrm{~s}, 3 \mathrm{H}), 3.69(\mathrm{~s}, 3 \mathrm{H}), 3.56(\mathrm{dd}, J=7.5$, $11.7 \mathrm{~Hz}, 1 \mathrm{H}), 3.42\left(\mathrm{dd}, J_{1}=8.7,11.7 \mathrm{~Hz}, 1 \mathrm{H}\right)$.

${ }^{13} \mathbf{C}$ NMR $\left(75 \mathrm{MHz}, \mathrm{CDCl}_{3}\right): \delta 161.5\left(\mathrm{~d}, J_{\mathrm{CF}}=243 \mathrm{~Hz}\right), 159.7,157.9,139.3,132.7,131.1$, $129.6\left(\mathrm{~d}, J_{\mathrm{CF}}=7.5 \mathrm{~Hz}\right), 129.0,128.3,126.8,124.5,115.1\left(\mathrm{~d}, J_{\mathrm{CF}}=21 \mathrm{~Hz}\right), 104.1,98.9$ 55.4, 55.3, 43.6, 32.9.

IR $\left(\mathrm{KBr}, \mathrm{cm}^{-1}\right): v$ 3060, 2995, 2940, 2835, 1599, 1502, 1458, 1291, 1210, 1152, 1033, 927 , $828,735,687$

HRMS (ESI ${ }^{+}$): calcd for $\left[\mathrm{C}_{22} \mathrm{H}_{22} \mathrm{FO}_{2} \mathrm{Se}\right]^{+}:$417.0769, found 417.0760.

\section{2,4,6-Trimethoxy-1-[1-(4-chlorophenyl)-2-(phenylseleno)ethyl]benzene (3ca)}

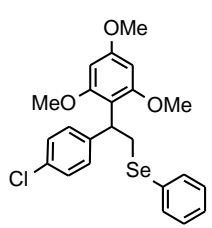

Yellow viscous liquid.

Yield: $169 \mathrm{mg}, 73 \%$.

${ }^{1}$ H NMR (300 MHz, $\mathrm{CDCl}_{3}$ ): $\delta$ 7.43-7.40 (m, 2H), 7.25-7.14 (m, 7H), $6.06(\mathrm{~s}, 2 \mathrm{H}), 4.88$ $(\mathrm{t}, J=8.4 \mathrm{~Hz}, 1 \mathrm{H}), 3.79(\mathrm{dd}, J=9.0,11.4 \mathrm{~Hz}, 1 \mathrm{H}), 3.72(\mathrm{~s}, 3 \mathrm{H}), 3.68(\mathrm{dd}, J=9.9,14.1$ $\mathrm{Hz}, 1 \mathrm{H}), 3.64(\mathrm{~s}, 6 \mathrm{H})$.

${ }^{13}$ C NMR $\left(75 \mathrm{MHz}, \mathrm{CDCl}_{3}\right): \delta 160.1,159.0,142.8,132.7,131.2,131.1,129.4,128.8$, $127.8,126.5,112.0,91.2,55.6,55.2,39.7,31.2$.

IR $\left(\mathrm{KBr}, \mathrm{cm}^{-1}\right): v$ 3056, 2995, 2942, 2831, 1597, 1468, 1413, 1328, 1205, 1144, 1110 , 1058, 1021, 948, 809, 731, 686.

HRMS (ESI ${ }^{+}$: calcd for $\left[\mathrm{C}_{23} \mathrm{H}_{24} \mathrm{ClO}_{3} \mathrm{Se}\right]^{+}$: 463.0579 , found 463.0572 .

4-Methoxy-2,3,6-trimethyl-1-[1-(4-chlorophenyl)-2-(phenylseleno)ethyl]benzene (3cb)

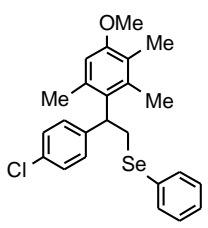

Yellow viscous liquid.

Yield: $166 \mathrm{mg}, 75 \%$.

${ }^{1}$ H NMR $\left(300 \mathrm{MHz}, \mathrm{CDCl}_{3}\right): \delta \quad 7.45-7.42(\mathrm{~m}, 2 \mathrm{H}), 7.23-7.20(\mathrm{~m}, 5 \mathrm{H}), 7.10(\mathrm{~d}, J=8.1$ $\mathrm{Hz}, 2 \mathrm{H}), 6.52(\mathrm{~s}, 1 \mathrm{H}), 4.78(\mathrm{t}, J=7.5 \mathrm{~Hz}, 1 \mathrm{H}), 3.82(\mathrm{dd}, J=7.5,11.7 \mathrm{~Hz}, 1 \mathrm{H}), 3.79$ (s, $3 \mathrm{H}), 3.55\left(\mathrm{dd}, J_{1}=8.1,11.7 \mathrm{~Hz}, 1 \mathrm{H}\right), 2.17-1.89(\mathrm{br}, 9 \mathrm{H})$. 
${ }^{13}$ C NMR $\left(75 \mathrm{MHz}, \mathrm{CDCl}_{3}\right): \delta 156.1,142.4,136.9,135.1,133.2,132.2,131.6,130.8$, $129.1,128.6,128.4,127.2,110.7,55.4,44.1,31.5,22.0,17.5,12.1$.

IR $\left(\mathrm{KBr}, \mathrm{cm}^{-1}\right): v$ 3052, 2936, 1582, 1477, 1385, 1301, 1193, 1115, 1009, 813, 736, 682. HRMS (ESI ${ }^{+}$: calcd for $\left[\mathrm{C}_{24} \mathrm{H}_{26} \mathrm{ClOSe}\right]^{+}: 445.0837$, found 445.0834 .

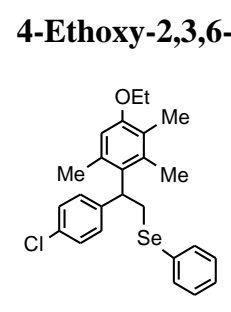

rimethyl-1-[1-(4-chlorophenyl)-2-(phenylseleno)ethyl]benzene (3cc)

Yellow viscous liquid.

Yield: $172 \mathrm{mg}, 75 \%$.

${ }^{1}$ H NMR (300 MHz, $\left.\mathrm{CDCl}_{3}\right): \delta 7.46-7.43(\mathrm{~m}, 2 \mathrm{H}), 7.23-7.20(\mathrm{~m}, 5 \mathrm{H}), 7.10(\mathrm{~d}, J=8.1$ $\mathrm{Hz}, 2 \mathrm{H}), 6.51(\mathrm{~s}, 1 \mathrm{H}), 4.78(\mathrm{t}, J=7.5 \mathrm{~Hz}, 1 \mathrm{H}), 3.99(\mathrm{q}, J=6.9 \mathrm{~Hz}, 2 \mathrm{H}), 3.82$ (dd, $J=$ $7.5,11.7 \mathrm{~Hz}, 1 \mathrm{H}), 3.55(\mathrm{dd}, J=7.8,11.7 \mathrm{~Hz}, 1 \mathrm{H}), 2.15-1.88(\mathrm{br}, 9 \mathrm{H}), 1.41(\mathrm{t}, J=6.9$ $\mathrm{Hz}, 3 \mathrm{H})$.

${ }^{13}$ C NMR (75 MHz, $\left.\mathrm{CDCl}_{3}\right): \delta 155.6,142.4,136.9,135.0,133.3,132.2,131.6,130.9$, $129.1,128.6,128.4,127.2,111.8,63.7,44.1,31.5,22.0,17.5,15.2,12.1$.

IR $\left(\mathrm{KBr}, \mathrm{cm}^{-1}\right): v$ 3060, 2974, 2925, 1582, 1480, 1389, 1302, 1113, 1005, 813, 737, 682 .

HRMS $\left(\mathrm{ESI}^{+}\right)$: calcd for $\left[\mathrm{C}_{25} \mathrm{H}_{28} \mathrm{ClOSe}\right]^{+}:$459.0994, found 459.0991 .

\section{5-Isopropyl-4-methoxy-2-methyl-1-[1-(4-chlorophenyl)-2-(phenylseleno)ethyl]benzene (3ce)}

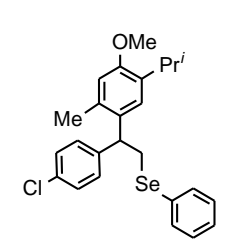

Yellow viscous liquid.

Yield: $163 \mathrm{mg}, 71 \%$.

${ }^{1}$ H NMR (300 MHz, $\left.\mathrm{CDCl}_{3}\right): \delta$ 7.46-7.43 (m, 2H), 7.24-7.20 (m, 5H), 7.09 (d, J = 7.2 $\mathrm{Hz}, 3 \mathrm{H}), 6.59(\mathrm{~s}, 1 \mathrm{H}), 4.34(\mathrm{t}, J=7.8 \mathrm{~Hz}, 1 \mathrm{H}), 3.77(\mathrm{~s}, 3 \mathrm{H}), 3.56-3.41(\mathrm{~m}, 2 \mathrm{H})$, $3.32-3.23(\mathrm{~m}, 1 \mathrm{H}), 2.10(\mathrm{~s}, 3 \mathrm{H}), 1.21(\mathrm{~d}, J=7.2 \mathrm{~Hz}, 3 \mathrm{H}), 1.18(\mathrm{~d}, J=7.2 \mathrm{~Hz}, 3 \mathrm{H})$.

${ }^{13}$ C NMR (75 MHz, $\left.\mathrm{CDCl}_{3}\right): \delta 155.4,142.4,134.6,134.4,133.1,132.7,132.2,130.8$, 129.6, 129.2, 128.7, 127.1, 124.4, 113.0, 55.4, 46.6, 34.3, 26.9, 22.9, 19.6.

IR $\left(\mathrm{KBr}, \mathrm{cm}^{-1}\right): v$ 3048, 2955, 1573, 1493, 1401, 1344, 1271, 1193, 1069, 1014, 890, $832,736,682$.

HRMS (ESI ${ }^{+}$): calcd for $\left[\mathrm{C}_{25} \mathrm{H}_{28} \mathrm{ClOSe}\right]^{+}:$459.0994, found 459.0990 .

2,4,6-Trimethoxy-1-[1-(4-bromophenyl)-2-(phenylseleno)ethyl]benzene (3da)

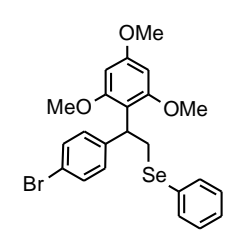

Yellow viscous liquid.

Yield: $230 \mathrm{mg}, 91 \%$.

${ }^{1} \mathbf{H}$ NMR $\left(300 \mathrm{MHz}, \mathrm{CDCl}_{3}\right): \delta 7.42-7.39(\mathrm{~m}, 2 \mathrm{H}), 7.30(\mathrm{~d}, J=8.4 \mathrm{~Hz}, 2 \mathrm{H}), 7.19-7.15$ $(\mathrm{m}, 5 \mathrm{H}), 6.05(\mathrm{~s}, 2 \mathrm{H}), 4.86(\mathrm{t}, J=8.1 \mathrm{~Hz}, 1 \mathrm{H}), 3.79(\mathrm{dd}, J=9,11.7 \mathrm{~Hz}, 1 \mathrm{H}), 3.72-3.67$ $(\mathrm{m}, 4 \mathrm{H}), 3.64(\mathrm{~s}, 6 \mathrm{H})$.

${ }^{13}$ C NMR (75 MHz, $\left.\mathrm{CDCl}_{3}\right): \delta 160.1,159.0,143.3,132.6,131.1,130.8,129.8,128.8$, 126.5, 119.4, 111.9, 91.1, 55.6, 55.2, 39.7, 31.1 .

IR $\left(\mathrm{KBr}, \mathrm{cm}^{-1}\right): v$ 3056, 2995, 2941, 2827, 1597, 1506, 1459, 1319, 1243, 1200, 1142, 1107, 1036, 948, 816, 735, 682.

HRMS $\left(\mathrm{ESI}^{+}\right)$: calcd for $\left[\mathrm{C}_{23} \mathrm{H}_{24} \mathrm{BrO}_{3} \mathrm{Se}\right]^{+}:$507.0074, found 507.0070. 


\section{4-Methoxy-2,3,6-trimethyl-1-[1-(4-bromophenyl)-2-(phenylseleno)ethyl]benzene (3db)}

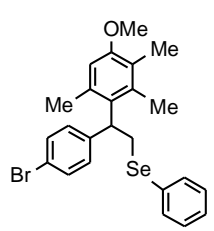

Yellow viscous liquid.

Yield: $212 \mathrm{mg}, 87 \%$.

${ }^{1} \mathbf{H}$ NMR $\left(300 \mathrm{MHz}, \mathrm{CDCl}_{3}\right): \delta 7.44-7.41(\mathrm{~m}, 2 \mathrm{H}), 7.34(\mathrm{~d}, J=8.4 \mathrm{~Hz}, 2 \mathrm{H}), 7.19-7.17$ $(\mathrm{m}, 3 \mathrm{H}), 7.04(\mathrm{~d}, J=8.1 \mathrm{~Hz}, 2 \mathrm{H}), 6.51(\mathrm{~s}, 1 \mathrm{H}), 4.76(\mathrm{t}, J=7.5 \mathrm{~Hz}, 1 \mathrm{H}), 3.80(\mathrm{dd}, J=$ 7.5, $11.7 \mathrm{~Hz}, 1 \mathrm{H}), 3.77$ (s, 3H), 3.54 (dd, $J=8.1,11.7 \mathrm{~Hz}, 1 \mathrm{H}), 2.16-1.88$ (br, 9H).

${ }^{13}$ C NMR (75 MHz, $\left.\mathrm{CDCl}_{3}\right): \delta 156.1,142.9,136.9,135.0,133.2,132.2,131.3,130.8$, 129.1, 129.0, 127.1, 119.6, 110.8, 55.4, 44.1, 31.4, 22.0, 17.5, 12.0.

IR $\left(\mathrm{KBr}, \mathrm{cm}^{-1}\right): v$ 3056, 2999, 2940, 2835, 1597, 1490, 1344, 1292, 1204, 1150, 1033, 922, 829, 737, 685 .

HRMS (ESI ${ }^{+}$): calcd for $\left[\mathrm{C}_{24} \mathrm{H}_{26} \mathrm{BrOSe}\right]^{+}$: 489.0332., found 489.0228.

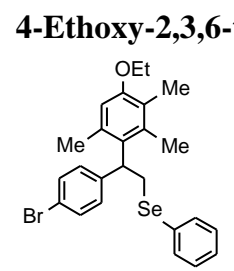

Yellow viscous liquid.

Yield: $191 \mathrm{mg}, 76 \%$.

${ }^{1}$ H NMR $\left(300 \mathrm{MHz}, \mathrm{CDCl}_{3}\right): \delta 7.44-7.42(\mathrm{~m}, 2 \mathrm{H}), 7.35(\mathrm{~d}, J=8.4 \mathrm{~Hz}, 2 \mathrm{H}), 7.21-7.19$ $(\mathrm{m}, 3 \mathrm{H}), 7.04(\mathrm{~d}, J=8.1 \mathrm{~Hz}, 2 \mathrm{H}), 6.51(\mathrm{~s}, 1 \mathrm{H}), 4.75(\mathrm{t}, J=7.2 \mathrm{~Hz}, 1 \mathrm{H}), 3.98(\mathrm{q}, J=6.6$ $\mathrm{Hz}, 2 \mathrm{H}), 3.81(\mathrm{dd}, J=7.5,11.7 \mathrm{~Hz}, 1 \mathrm{H}), 3.54\left(\mathrm{dd}, J_{1}=7.8,11.7 \mathrm{~Hz}, 1 \mathrm{H}\right), 2.15-1.88$ (br, $9 \mathrm{H}), 1.40(\mathrm{t}, J=6.9 \mathrm{~Hz}, 3 \mathrm{H})$.

${ }^{13}$ C NMR (75 MHz, $\left.\mathrm{CDCl}_{3}\right): \delta 155.5,143.0,136.9,134.9,133.2,132.1,131.3,130.8$, $129.1,129.0,127.1,119.6,111.8,63.7,44.1,31.4,22.0,17.5,15.2,12.1$.

IR $\left(\mathrm{KBr}, \mathrm{cm}^{-1}\right): v$ 3060, 2974, 2926, 1581, 1476, 1388, 1299, 1218, 1111, 1014, 903, 810 $739,688$.

HRMS $\left(\mathrm{ESI}^{+}\right)$: calcd for $\left[\mathrm{C}_{25} \mathrm{H}_{28} \mathrm{BrOSe}\right]^{+}:$503.0489, found 503.0481.

\section{5-Isopropyl-4-methoxy-2-methyl-1-[1-(4-bromophenyl)-2-(phenylseleno)ethyl]benzene (3de)}

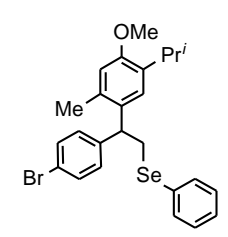

Yellow viscous liquid.

Yield: $171 \mathrm{mg}, 68 \%$.

${ }^{1}$ H NMR $\left(300 \mathrm{MHz}, \mathrm{CDCl}_{3}\right): \delta 7.46-7.42(\mathrm{~m}, 2 \mathrm{H}), 7.36(\mathrm{~d}, J=7.8 \mathrm{~Hz}, 2 \mathrm{H}), 7.23-7.19$ (m, 3H), $7.10(\mathrm{~s}, 1 \mathrm{H}), 7.03(\mathrm{~d}, J=8.4 \mathrm{~Hz}, 2 \mathrm{H}), 6.59$ (s, 1H), $4.32(\mathrm{t}, J=8.0 \mathrm{~Hz}, 1 \mathrm{H}), 3.77$ (s, 3H), 3.56-3.41 (m, 2H), 3.32-3.23 (m, 1H), $2.1(\mathrm{~s}, 3 \mathrm{H}), 1.21(\mathrm{~d}, J=6.9 \mathrm{~Hz}, 3 \mathrm{H}), 1.20$ (d, $J=6.9 \mathrm{~Hz}, 3 \mathrm{H})$.

${ }^{13}$ C NMR $\left(75 \mathrm{MHz}, \mathrm{CDCl}_{3}\right): \delta 155.4,142.8,134.5,134.4,133.0,132.5,131.6,130.8$, $129.9,129.2,127.1,124.3,120.3,112.9,55.4,46.6,34.2,26.9,22.9,19.7$.

IR $\left(\mathrm{KBr}, \mathrm{cm}^{-1}\right): v$ 3052, 2954, 1610, 1574, 1491, 1458, 1399, 1349, 1267, 1192, 1065, 1010, 898, 821, 736, 690 .

HRMS (ESI ${ }^{+}$): calcd for $\left[\mathrm{C}_{25} \mathrm{H}_{28} \mathrm{BrOSe}\right]^{+}$: 503.0489, found 503.0483.

2,4-Dimethoxy-1-[1-(4-bromophenyl)-2-(phenylseleno)ethyl]benzene (3df) 


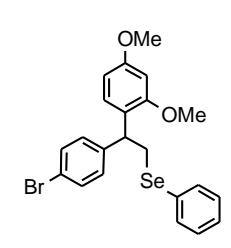

Yellow viscous liquid.

Yield: $229 \mathrm{mg}, 96 \%$.

${ }^{1} \mathbf{H}$ NMR $\left(300 \mathrm{MHz}, \mathrm{CDCl}_{3}\right): \delta 7.46-7.43(\mathrm{~m}, 2 \mathrm{H}), 7.36(\mathrm{~d}, J=8.4 \mathrm{~Hz}, 2 \mathrm{H}), 7.22-7.19$ (m, 3H), 7.09 (d, $J=8.4 \mathrm{~Hz}, 2 \mathrm{H}), 6.98$ (d, $J=9 \mathrm{~Hz}, 1 \mathrm{H}), 6.42-6.39(\mathrm{~m}, 2 \mathrm{H}), 4.56$ (t, $J=$ $7.8 \mathrm{~Hz}, 1 \mathrm{H}), 3.74$ (s, 3H), 3.68 (s, 3H), 3.55 (dd, $J=7.5,11.7 \mathrm{~Hz}, 1 \mathrm{H}), 3.41(\mathrm{dd}, J=8.7$, $11.7 \mathrm{~Hz}, 1 \mathrm{H})$.

${ }^{13}$ C NMR $\left(75 \mathrm{MHz}, \mathrm{CDCl}_{3}\right): \delta$ 159.8, 157.9, 142.7, 132.8, 131.4, 131.0, 130.0, 129.0, $128.3,126.8,124.1,120.2,104.2,98.9,55.4,43.9,32.6$.

IR $\left(\mathrm{KBr}, \mathrm{cm}^{-1}\right): v$ 3056, 2940, 1583, 1477, 1389, 1303, 1193, 1119, 1070, 1009, 805, 737, 686.

HRMS (ESI ${ }^{+}$): calcd for $\left[\mathrm{C}_{22} \mathrm{H}_{22} \mathrm{BrO}_{2} \mathrm{Se}\right]^{+}: 476.9968$, found 476.9968.

\section{2,4,6-Trimethoxy-1-[1-(4-methylphenyl)-2-(phenylseleno)ethyl]benzene (3ea)}

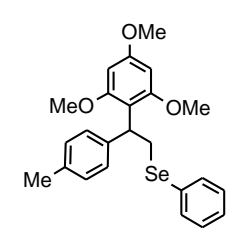

Yellow viscous liquid.

Yield: $170 \mathrm{mg}, 77 \%$.

${ }^{1}$ H NMR $\left(300 \mathrm{MHz}, \mathrm{CDCl}_{3}\right): \delta 7.44-7.41(\mathrm{~m}, 2 \mathrm{H}), 7.22-7.17(\mathrm{~m}, 5 \mathrm{H}), 7.02(\mathrm{~d}, J=7.8$ $\mathrm{Hz}, 2 \mathrm{H}), 6.07$ (s, 2H), 4.89 (dd, $J=7.2,9.3 \mathrm{~Hz}, 1 \mathrm{H}), 3.88(\mathrm{dd}, J=9.6,11.4 \mathrm{~Hz}, 1 \mathrm{H}), 3.78$ (s, 3H), $3.70(\mathrm{dd}, J=7.5,11.7 \mathrm{~Hz}, 1 \mathrm{H}), 3.68(\mathrm{~s}, 6 \mathrm{H}), 2.26$ (s, 3H).

${ }^{13}$ C NMR (75 MHz, $\left.\mathrm{CDCl}_{3}\right): \delta 159.9,159.3,141.3,135.2,132.6,131.6,128.8,128.6$, $127.8,126.4,112.5,91.2,55.7,55.3,39.9,31.8,21.1$.

IR $\left(\mathrm{KBr}, \mathrm{cm}^{-1}\right): v$ 3052, 3003, 2938, 2840, 1596, 1459, 1324, 1205, 1140, 1050, 948, 809, 735,682 .

HRMS $\left(\mathrm{ESI}^{+}\right)$: calcd for $\left[\mathrm{C}_{24} \mathrm{H}_{26} \mathrm{O}_{3} \mathrm{NaSe}\right]^{+}:$465.0945, found 465.0943 .

\section{4-Methoxy-2,3,6-trimethyl-1-[1-(4-methylphenyl)-2-(phenylseleno)ethyl]benzene (3eb)}

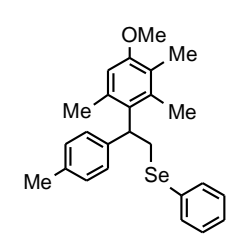

Yellow viscous liquid.

Yield: $163 \mathrm{mg}, 77 \%$.

${ }^{1}$ H NMR (300 MHz, $\left.\mathrm{CDCl}_{3}\right): \delta 7.46-7.43(\mathrm{~m}, 2 \mathrm{H}), 7.22-7.19(\mathrm{~m}, 3 \mathrm{H}), 7.06(\mathrm{~s}, 4 \mathrm{H}), 6.52$ $(\mathrm{s}, 1 \mathrm{H}), 4.83(\mathrm{t}, J=7.8 \mathrm{~Hz}, 1 \mathrm{H}), 3.88(\mathrm{dd}, J=7.2,11.7 \mathrm{~Hz}, 1 \mathrm{H}), 3.79(\mathrm{~s}, 3 \mathrm{H}), 3.58$ (dd, $J_{1}$ $=8.4,11.7 \mathrm{~Hz}, 1 \mathrm{H}), 2.30(\mathrm{~s}, 3 \mathrm{H}), 2.19-1.93(\mathrm{br}, 9 \mathrm{H})$.

${ }^{13}$ C NMR (75 MHz, $\left.\mathrm{CDCl}_{3}\right): \delta$ 155.9, 140.8, 137.1, 135.3, 135.2, 133.0, 132.9, 131.3, $129.1,127.0,126.9,110.7,55.5,44.2,31.8,22.1,21.1,17.5,12.1$.

IR $\left(\mathrm{KBr}, \mathrm{cm}^{-1}\right): v$ 2999, 2934, 1583, 1462, 1300, 1201, 1117, 1018, 903, 812, 744, 686.

HRMS $\left(\mathrm{ESI}^{+}\right)$: calcd for $\left[\mathrm{C}_{25} \mathrm{H}_{29} \mathrm{OSe}\right]^{+}: 425.1384$, found 425.1379.

\section{2,4-Dimethoxy-1-[1-(4-methylphenyl)-2-(phenylseleno)ethyl]benzene (3ef)}<smiles>COc1ccc(C(Cc2ccccc2)c2ccc(C)cc2)c(OC)c1</smiles>

Yellow viscous liquid.

Yield: $136 \mathrm{mg}, 66 \%$.

${ }^{1}$ H NMR $\left(300 \mathrm{MHz}, \mathrm{CDCl}_{3}\right): \delta$ 7.47-7.44 (m, 2H), 7.21-6.99 (m, 8H), 6.40-6.37 (m, $2 \mathrm{H}), 4.61(\mathrm{t}, J=8.4 \mathrm{~Hz}, 1 \mathrm{H}), 3.71(\mathrm{~s}, 3 \mathrm{H}), 3.67(\mathrm{~s}, 3 \mathrm{H}), 3.60-3.42(\mathrm{~m}, 2 \mathrm{H}), 2.27(\mathrm{~s}, 3 \mathrm{H})$

${ }^{13} \mathrm{C}$ NMR $\left(75 \mathrm{MHz}, \mathrm{CDCl}_{3}\right): \delta 159.5,157.9,140.5,135.8,132.4,131.4,129.1,128.9$, $128.4,128.0,126.5,124.8,104.0,98.7,55.3,55.3,43.7,32.9,21.1$.

IR $\left(\mathrm{KBr}, \mathrm{cm}^{-1}\right): v$ 3050, 2935, 1580, 1473, 1385, 1190, 1118, 801.

HRMS $\left(\right.$ ESI $\left.^{+}\right)$: calcd for $\left[\mathrm{C}_{23} \mathrm{H}_{25} \mathrm{O}_{2} \mathrm{Se}\right]^{+}$: 413.1020 , found 413.1018 . 
2,4,6-Trimethoxy-1-[1-(4-methoxyphenyl)-2-(phenylseleno)ethyl]benzene (3fa)

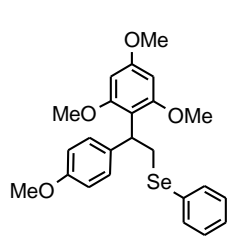

Yellow viscous liquid.

Yield: $178 \mathrm{mg}, 78 \%$.

${ }^{1}$ H NMR $\left(300 \mathrm{MHz}, \mathrm{CDCl}_{3}\right): \delta 7.44-7.41(\mathrm{~m}, 2 \mathrm{H}), 7.26-7.17(\mathrm{~m}, 5 \mathrm{H}), 6.76(\mathrm{~d}, J=8.7$ $\mathrm{Hz}, 2 \mathrm{H}), 6.08(\mathrm{~s}, 2 \mathrm{H}), 4.87(\mathrm{t}, J=8.4 \mathrm{~Hz}, 1 \mathrm{H}), 3.85(\mathrm{dd}, J=9.6,11.1 \mathrm{~Hz}, 1 \mathrm{H})$, 3.79-3.69 (m, 13H).

${ }^{13}$ C NMR (75 MHz, $\left.\mathrm{CDCl}_{3}\right): \delta 159.9,159.2,157.7,136.5,132.6,131.5,128.9,128.8$, $126.4,113.3,112.6,91.2,55.7,55.3,55.2,39.6,31.9$.

IR $\left(\mathrm{KBr}, \mathrm{cm}^{-1}\right): v$ 3056, 2995, 2941, 2827, 1597, 1506, 1459, 1319, 1243, 1200, 1142 1107, 1036, 948, 816, 735, 682 .

HRMS $\left(\mathrm{ESI}^{+}\right)$: calcd for $\left[\mathrm{C}_{24} \mathrm{H}_{26} \mathrm{O}_{4} \mathrm{Se}\right]^{+}: 458.0996$, found 458.0996 .

4-Ethoxy-2,3,6-trimethyl-1-[1-(4-nitrophenyl)-2-(phenylseleno)ethyl]benzene (3gc)

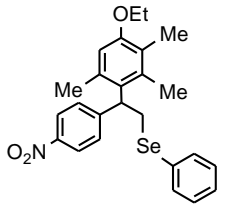

Yellow viscous liquid.

Yield: $206 \mathrm{mg}, 88 \%$

${ }^{1}$ H NMR $\left(300 \mathrm{MHz}, \mathrm{CDCl}_{3}\right): \delta 8.10(\mathrm{~d}, J=8.7 \mathrm{~Hz}, 2 \mathrm{H}), 7.47-7.44(\mathrm{~m}, 2 \mathrm{H}), 7.34(\mathrm{~d}$, $J=8.4 \mathrm{~Hz}, 2 \mathrm{H}), 7.24-7.20(\mathrm{~m}, 3 \mathrm{H}), 6.53(\mathrm{~s}, 1 \mathrm{H}), 4.84(\mathrm{t}, J=7.2 \mathrm{~Hz}, 1 \mathrm{H}), 3.97$ (q, $J=$ $6.9 \mathrm{~Hz}, 2 \mathrm{H}), 3.85(\mathrm{dd}, J=7.8,12 \mathrm{~Hz}, 1 \mathrm{H}), 3.57(\mathrm{dd}, J=7.5,12 \mathrm{~Hz}, 1 \mathrm{H}), 2.18-1.84$ (br, 9H), $1.41(\mathrm{t}, J=7.2 \mathrm{~Hz}, 3 \mathrm{H})$.

${ }^{13} \mathbf{C}$ NMR $\left(75 \mathrm{MHz}, \mathrm{CDCl}_{3}\right): \delta 155.8,152.0,146.1,136.7,134.8,133.5,131.6,130.3$, $129.2,128.1,127.4,123.5,112.0,63.7,44.9,31.0,21.9,17.6,15.2,12.1$.

IR $\left(\mathrm{KBr}, \mathrm{cm}^{-1}\right): v$ 3060, 2974, 2926, 2868, 1590, 1520, 1477, 1387, 1344, 1305, 1115, $1025,852,739,699,537,481$.

HRMS (ESI $\left.{ }^{+}\right)$: calcd for $\left[\mathrm{C}_{25} \mathrm{H}_{27} \mathrm{NNaO}_{3} \mathrm{Se}\right]^{+}$: 492.1054 , found 492.1053 .

\section{2,4,6-Trimethoxy-1-[1-phenyl-2-(phenylseleno)propyl]benzene (3ha)}

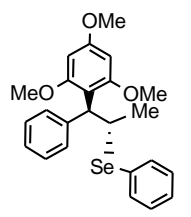

Yellow viscous liquid.

Yield: $128 \mathrm{mg}, 58 \%$.

${ }^{1}$ H NMR $\left(300 \mathrm{MHz}, \mathrm{CDCl}_{3}\right): \delta 7.52-7.47(\mathrm{~m}, 4 \mathrm{H}), 7.27-7.12(\mathrm{~m}, 6 \mathrm{H}), 6.05(\mathrm{~s}, 2 \mathrm{H})$, $4.70(\mathrm{~d}, J=11.7 \mathrm{~Hz}, 1 \mathrm{H}), 4.60-4.49(\mathrm{~m}, 1 \mathrm{H}), 3.76(\mathrm{~s}, 6 \mathrm{H}), 3.74(\mathrm{~s}, 3 \mathrm{H}), 1.29$ (d, $J=$ $6.6 \mathrm{~Hz}, 3 \mathrm{H})$

${ }^{13}$ C NMR $\left(75 \mathrm{MHz}, \mathrm{CDCl}_{3}\right): \delta 159.8,158.9,144.0,135.8,130.0,128.9,128.7,127.7$, 127.3, 126.0, 112.8, 91.2, 55.8, 55.3, 47.1, 41.3, 22.0.

IR $\left(\mathrm{KBr}, \mathrm{cm}^{-1}\right): v 2925,2844,1592,1454,1328,1209,1119,1054,943,805,743$, 698.

HRMS $\left(\mathrm{ESI}^{+}\right)$: calcd for $\left[\mathrm{C}_{24} \mathrm{H}_{26} \mathrm{NaO}_{3} \mathrm{Se}\right]^{+}:$465.0945, found 465.0940 . 


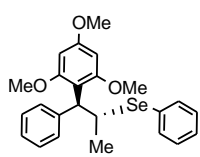

Yellow viscous liquid.

Yield: $143 \mathrm{mg}, 65 \%$

${ }^{1} \mathbf{H}$ NMR $\left(300 \mathrm{MHz}, \mathrm{CDCl}_{3}\right): \delta$ 7.48-7.44 (m, 4H), 7.21-7.06 (m, 6H), $6.06(\mathrm{~s}, 2 \mathrm{H})$, $4.82-4.71(\mathrm{~m}, 1 \mathrm{H}), 4.57(\mathrm{~d}, J=12 \mathrm{~Hz}, 1 \mathrm{H}), 3.79(\mathrm{~s}, 6 \mathrm{H}), 3.73(\mathrm{~s}, 3 \mathrm{H}), 1.33$ (d, $J=6.6$ $\mathrm{Hz}, 3 \mathrm{H})$.

${ }^{13}$ C NMR $\left(75 \mathrm{MHz}, \mathrm{CDCl}_{3}\right): \delta 159.8,158.9,143.9,135.6,129.8,128.6,128.6,128.0$, $127.2,125.9,113.4,91.1,55.7,55.2,48.2,41.6,22.6$

IR $\left(\mathrm{KBr}, \mathrm{cm}^{-1}\right): v 3056,2938,2835,1597,1457,1324,1205,1126,1054,948,805$, $739,690$.

HRMS $\left(\mathrm{ESI}^{+}\right)$: calcd for $\left[\mathrm{C}_{24} \mathrm{H}_{26} \mathrm{NaO}_{3} \mathrm{Se}\right]^{+}:$465.0945, found 465.0938 .

\section{2,4,6-Trimethoxy-1-[(2-phenylseleno)cyclohexyl]benzene ( $( \pm-3 \mathbf{j a})$}

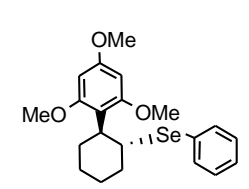

Yellow viscous liquid.

Yield: $136 \mathrm{mg}, 67 \%$.

${ }^{1} \mathbf{H}$ NMR $\left(300 \mathrm{MHz}, \mathrm{CDCl}_{3}\right): \delta 7.37-7.34(\mathrm{~m}, 2 \mathrm{H}), 7.17-7.11(\mathrm{~m}, 3 \mathrm{H}), 6.08-6.06(\mathrm{~m}$, $2 \mathrm{H}), 4.13-4.04(\mathrm{~m}, 1 \mathrm{H}), 3.76(\mathrm{~s}, 6 \mathrm{H}), 3.75(\mathrm{~s}, 3 \mathrm{H}), 3.38-3.29(\mathrm{~m}, 1 \mathrm{H}), 2.20-1.26(\mathrm{~m}$ $8 \mathrm{H})$.

${ }^{13}$ C NMR (75 MHz, $\left.\mathrm{CDCl}_{3}\right): \delta 161.6,159.3,135.2,130.0,128.4,126.7,113.9,92.9$, 91.0, 55.4, 55.2, 46.9, 40.8, 36.3, 31.9, 27.9, 26.8 .

IR $\left(\mathrm{KBr}, \mathrm{cm}^{-1}\right): v$ 3056, 2995, 2927, 2846, 1599, 1457, 1373, 1324, 1204, 1152, 1121 , $1066,948,809,735,686$.

HRMS $\left(\mathrm{ESI}^{+}\right)$: calcd for $\left[\mathrm{C}_{21} \mathrm{H}_{26} \mathrm{NaO}_{3} \mathrm{Se}\right]^{+}:$429.0945, found 429.0941

\section{(E)-4-methoxy-2,3,6-trimethyl-1-[1-phenyl-2-(phenylseleno)ethenyl]benzene (7b)}

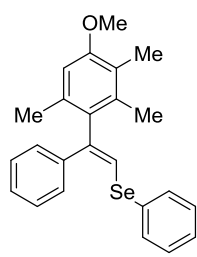

Yellow viscous liquid.

Yield: $122 \mathrm{mg}, 60 \%$.

${ }^{1}$ H NMR $\left(300 \mathrm{MHz}, \mathrm{CDCl}_{3}\right): \delta 7.57-7.54(\mathrm{~m}, 2 \mathrm{H}), 7.36-7.22(\mathrm{~m}, 8 \mathrm{H}), 6.59(\mathrm{~s}$, $1 \mathrm{H}), 6.55(\mathrm{~s}, 1 \mathrm{H}), 3.80(\mathrm{~s}, 3 \mathrm{H}), 2.21(\mathrm{~s}, 3 \mathrm{H}), 2.17$ (s, 3H), $2.13(\mathrm{~s}, 3 \mathrm{H})$.

${ }^{13} \mathrm{C}$ NMR $\left(75 \mathrm{MHz}, \mathrm{CDCl}_{3}\right): \delta 156.6,141.6,140.0,136.6,135.2,134.5,132.8$, 132.2 , 129.3, 128.3, 128.1, 127.6, 127.5, 123.0, 122.7, 109.7, 55.6, 21.0, 17.7, 12.0.

IR $\left(\mathrm{KBr}, \mathrm{cm}^{-1}\right): v 3056,2921,2855,1581,1464,1377,1307,1120,1017,833$, 739,695 .

HRMS (ESI ${ }^{+}$): calcd for $\left[\mathrm{C}_{24} \mathrm{H}_{25} \mathrm{OSe}\right]^{+}$: 409.1071, found 409.1076 .

\section{(E)-4-Ethoxy-2,3,6-trimethyl-1-[1-phenyl-2-(phenylseleno)ethenyl]benzene (7c)}

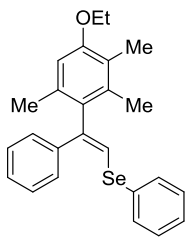

Yellow viscous liquid.

Yield: $122 \mathrm{mg}, 58 \%$.

${ }^{1}$ H NMR $\left(300 \mathrm{MHz}, \mathrm{CDCl}_{3}\right): \delta$ 7.56-7.54 (m, 2H), 7.36-7.19 (m, 8H), 6.57 (s, 1H), 6.55 (s, 1H), 4.00 (q, J = 6.9 Hz, 2H), 2.19 (s, 3H), $2.16(\mathrm{~s}, 3 \mathrm{H}), 2.14$ (s, 3H), $1.41(\mathrm{t}, J=6.9 \mathrm{~Hz}, 3 \mathrm{H})$.

${ }^{13}$ C NMR (75 MHz, $\left.\mathrm{CDCl}_{3}\right): \delta 156.0,141.6,140.1,136.5,135.1,134.4,132.8$, 132.2, 129.3, 128.3, 128.1, 127.5, 127.5, 122.9, 110.8, 63.9, 21.1, 17.7, 15.2, 12.1 . IR $\left(\mathrm{KBr}, \mathrm{cm}^{-1}\right): v$ 3058, 2966, 2921, 2864, 1581, 1473, 1386, 1310, 1228, 1156, 
1112, 1024, 919, 819, 741, 693.

HRMS $\left(\mathrm{ESI}^{+}\right)$: calcd for $\left[\mathrm{C}_{25} \mathrm{H}_{27} \mathrm{OSe}\right]^{+}:$423.1227, found 423.1234 .

\section{4-Methoxy-2,3,6-trimethyl-1-(1-phenylethenyl)benzene (5ab)}

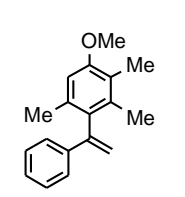

Light yellow solid.

Mp: $87.3-88.5^{\circ} \mathrm{C}$.

Yield: $46 \mathrm{mg}, 91 \%$.

${ }^{1} \mathbf{H}$ NMR (300 MHz, $\left.\mathrm{CDCl}_{3}\right): \delta 7.29-7.20(\mathrm{~m}, 5 \mathrm{H}), 6.63(\mathrm{~s}, 1 \mathrm{H}), 5.97(\mathrm{~d}, J=1.5 \mathrm{~Hz}, 1 \mathrm{H})$, $5.07(\mathrm{~d}, J=1.2 \mathrm{~Hz}, 1 \mathrm{H}), 3.83(\mathrm{~s}, 3 \mathrm{H}), 2.16(\mathrm{~s}, 3 \mathrm{H}), 2.13(\mathrm{~s}, 3 \mathrm{H}), 2.07(\mathrm{~s}, 3 \mathrm{H})$.

${ }^{13}$ C NMR $\left(75 \mathrm{MHz}, \mathrm{CDCl}_{3}\right): \delta 156.4,147.6,140.2,136.2,134.0,133.7,128.5,127.6$, 126.1, 122.4, 114.9, 109.5, 55.6, 20.8, 17.3, 12.0.

IR $\left(\mathrm{KBr}, \mathrm{cm}^{-1}\right): v 2920,1586,1482,1310,1182,1117,902,845,782,708,474 \mathrm{~cm}^{-1}$.

HRMS $\left(\mathrm{ESI}^{+}\right)$: calcd for $\left[\mathrm{C}_{18} \mathrm{H}_{21} \mathrm{O}\right]^{+}:$253.1592, found 253.1593 .

\section{(Z)-1-(1-phenyl-1-propenyl)-2,4,6-trimethoxybenzene (5ha)}

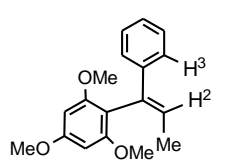

Yellowish liquid.

Yield: $52 \mathrm{mg}, 92 \%$.

${ }^{1} \mathbf{H}$ NMR $\left(400 \mathrm{MHz}, \mathrm{CDCl}_{3}\right): \delta 7.25-7.18(\mathrm{~m}, 4 \mathrm{H}), 7.15-7.11(\mathrm{~m}, 1 \mathrm{H}), 6.34(\mathrm{q}, J=6.8$ $\mathrm{Hz}, 1 \mathrm{H}), 6.21$ (s, 2H), 3.85 (s, 3H), 3.68 (s, 6H), 1.60 (d, J=6.8 Hz, 3H).

${ }^{13}$ C NMR (100 MHz, $\left.\mathrm{CDCl}_{3}\right): \delta 160.8,158.8,142.1,134.4,128.1,126.3,125.9,125.7$, 109.8, 91.0, 56.0, 55.4, 15.6 .

IR $\left(\mathrm{KBr}, \mathrm{cm}^{-1}\right): v 2843,1662,1610,1402,1335,1213,1127,1061,947,769,689,585$ $\mathrm{cm}^{-1}$.

HRMS $\left(\mathrm{ESI}^{+}\right)$: calcd for $\left[\mathrm{C}_{18} \mathrm{H}_{20} \mathrm{O}_{3}\right]^{+}: 284.1412$, found 284.1415 .

\section{(E)-1-(1-phenyl-1-propenyl)-2,4,6-trimethoxybenzene (5ia)}

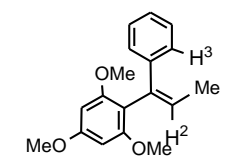

Yellowish liquid.

Yield: $51 \mathrm{mg}, 90 \%$.

${ }^{1} \mathbf{H}$ NMR $\left(400 \mathrm{MHz}, \mathrm{CDCl}_{3}\right): \delta 7.26-7.24(\mathrm{~m}, 4 \mathrm{H}), 7.17-7.13(\mathrm{~m}, 1 \mathrm{H}), 6.13(\mathrm{~s}, 2 \mathrm{H}), 5.69$ (q, $J=6.8 \mathrm{~Hz}, 1 \mathrm{H}), 3.80(\mathrm{~s}, 3 \mathrm{H}), 3.69(\mathrm{~s}, 6 \mathrm{H}), 1.85(\mathrm{~d}, J=6.8 \mathrm{~Hz}, 3 \mathrm{H})$.

${ }^{13} \mathbf{C}$ NMR (100 MHz, $\left.\mathrm{CDCl}_{3}\right): \delta 160.2,158.8,141.4,133.9,129.0,127.6,127.5,126.0$, 115.9, 91.1, 56.1, 55.4, 15.4 .

IR $\left(\mathrm{KBr}, \mathrm{cm}^{-1}\right): v$ 2921, 2849, 1610, 1453, 1402, 1208, 1124, 1057, 778, 700, $589 \mathrm{~cm}^{-1}$. HRMS $\left(\right.$ ESI $\left.^{+}\right)$: calcd for $\left[\mathrm{C}_{18} \mathrm{H}_{20} \mathrm{O}_{3}\right]^{+}: 284.1412$, found 284.1420 .

4-Ethoxy-2,3,6-trimethyl-1-(1-phenylethenyl)benzene (8c)

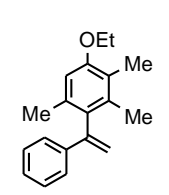

Light yellow solid.

Mp: $92.5-94.1^{\circ} \mathrm{C}$.

Yield: $48 \mathrm{mg}, 90 \%$.

${ }^{1}$ H NMR (300 MHz, $\left.\mathrm{CDCl}_{3}\right): \delta 7.28-7.22(\mathrm{~m}, 5 \mathrm{H}), 6.62(\mathrm{~s}, 1 \mathrm{H}), 5.96(\mathrm{~d}, J=1.2 \mathrm{~Hz}, 1 \mathrm{H})$, $5.07(\mathrm{~d}, J=1.2 \mathrm{~Hz}, 1 \mathrm{H}), 4.08-4.00(\mathrm{qd}, J=6.9 \mathrm{~Hz}, 1.2 \mathrm{~Hz}, 2 \mathrm{H}), 2.16(\mathrm{~s}, 3 \mathrm{H}), 2.11(\mathrm{~s}, 3 \mathrm{H})$, 
2.07 (s, 3H), 1.44 (t, $J=6.9 \mathrm{~Hz}, 3 \mathrm{H})$.

${ }^{13} \mathrm{C}$ NMR $\left(75 \mathrm{MHz}, \mathrm{CDCl}_{3}\right): \delta 155.9,147.7,140.3,136.2,133.9,133.6,128.5,127.6$, $126.1,122.7,114.9,110.8,63.9,20.8,17.3,15.3,12.1$.

IR $\left(\mathrm{KBr}, \mathrm{cm}^{-1}\right): v$ 2933, 1578, 1486, 1330, 1178, 1109, 904, 847, 779, 708, $474 \mathrm{~cm}^{-1}$.

HRMS $\left(\mathrm{ESI}^{+}\right)$: calcd for $\left[\mathrm{C}_{19} \mathrm{H}_{23} \mathrm{O}\right]^{+}: 267.1749$, found 267.1749 .

(2R)-Methyl-2-[(2-phenyl-2-(2,4,6-trimethoxyphenyl)ethyl-1-seleno-(2,1-phenyleneoxy)]propionate $\left(3 \mathbf{a k}+3 \mathbf{a k}^{\prime}\right)$

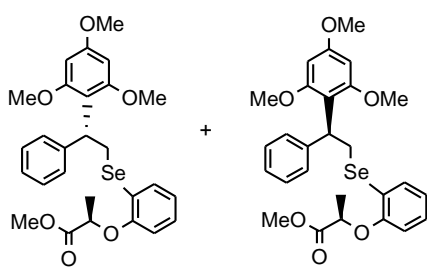

Yellow viscous liquid.

Yield: $307 \mathrm{mg}$, 58\%.

${ }^{1}$ H NMR $\left(300 \mathrm{MHz}, \mathrm{CDCl}_{3}\right): \delta 7.52-6.66(\mathrm{~m}, 9 \mathrm{H}), 6.10(\mathrm{~s}, 2 \mathrm{H}), 4.94(\mathrm{t}$, $J=8.1 \mathrm{~Hz}, 1 \mathrm{H}), 4.83-4.68(\mathrm{~m}, 1 \mathrm{H}), 3.82-3.68(\mathrm{~m}, 14 \mathrm{H}), 1.61(\mathrm{~d}, J=6.6$ $\mathrm{Hz}, 3 \mathrm{H})$.

${ }^{13} \mathbf{C}$ NMR $\left(75 \mathrm{MHz}, \mathrm{CDCl}_{3}\right) \delta 172.7,160.0,159.4,156.3,144.6,132.0$, $128.1,127.9,127.1,125.7,122.5,122.3,113.1,113.0,91.4,73.9,55.8$, $55.3,52.3,40.0,29.0,18.7$.

IR $\left(\mathrm{KBr}, \mathrm{cm}^{-1}\right): v 3060,2995,2940,2840,1749,1600,1460,1282$, 1209, 1142, 1106, 1047, 952, 912, 811, 739, 540, 452.

HRMS (ESI ${ }^{+}$): calcd for $\left[\mathrm{C}_{27} \mathrm{H}_{30} \mathrm{NaO}_{6} \mathrm{Se}\right]^{+}: 553.1105$, found 553.1103 .

(2R)-Methyl-2-[(2-phenyl-2-(4-ethoxy-2,3,6-trimethylphenyl)ethyl-1-seleno-(2,1-phenyleneoxy)]propio nate $\left(3 \mathrm{ck}+3 \mathrm{ck}^{\prime}\right)$

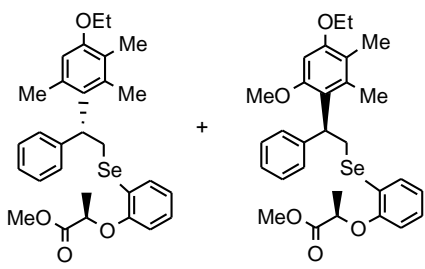

Yellow viscous liquid.

Yield: $363 \mathrm{mg}, 69 \%$.

${ }^{1}$ H NMR $\left(400 \mathrm{MHz}, \mathrm{CDCl}_{3}\right): \delta 7.38-7.36(\mathrm{~m}, 1 \mathrm{H}), 7.25-6.53(\mathrm{~m}, 9 \mathrm{H})$, 4.91-4.87 (m, 1H), 4.79-4.74 (m, 0.7H), 4.72-4.68 (m, 0.3H), 4.01-3.98 (m, 2H), 3.94-3.83 (m, 1H), 3.71-3.58 (m, 4H), 2.19-1.96 (br, 9H), $1.64-1.59(\mathrm{~m}, 3 \mathrm{H}), 1.43-1.40(\mathrm{~m}, 3 \mathrm{H})$.

${ }^{13}$ C NMR (100 MHz, $\left.\mathrm{CDCl}_{3}\right): \delta 172.6,156.4,155.4,144.2,143.9,137.1$, $135.1,134.5,132.9,132.8,130.2,128.4,128.3,127.9,127.2,127.1$, $125.8,125.7,124.0,122.5,121.6,114.6,114.1,112.8,112.0,73.9,73.6$, 63.7, 52.5, 52.4, 44.2, 44.1, 29.3, 29.2, 22.1, 18.8, 18.7, 17.6, 15.3, 12.2 .

IR $\left(\mathrm{KBr}, \mathrm{cm}^{-1}\right): v$ 3030, 2974, 2922, 2815, 1751, 1583, 1461, 1385, 1295, 1232, 1113, 1045, 923, 845, 746, 701, 442.

HRMS $\left(\right.$ ESI $^{+}$): calcd for $\left[\mathrm{C}_{29} \mathrm{H}_{34} \mathrm{NaO}_{5} \mathrm{Se}^{+}:\right.$565.1469, found 565.1465.

\section{The data summarized from HSQC and HMBC Spectra for compound 3ae}

Table 2. The Data Summarized from the HSQC Spectrum of 3ae

\begin{tabular}{cccc} 
Entry & $\delta_{\mathrm{C}} / \mathrm{ppm}$ & Order of $\mathrm{C}$ atoms & Directly connected $\mathrm{H}, \delta_{\mathrm{H}} / \mathrm{ppm}$ \\
1 & 155.1 & $\mathrm{C}$ \\
2 & 143.8 & $\mathrm{C}$ \\
3 & 134.4 & $\mathrm{C}$ \\
\hline
\end{tabular}




\begin{tabular}{cccc}
4 & 134.2 & $\mathrm{C}$ & \\
5 & 132.9 & $\mathrm{C}$ & 7.44 \\
6 & 132.8 & $2 \mathrm{CH}$ & \\
7 & 131.1 & $\mathrm{C}$ & 7.22 \\
8 & 129.0 & $2 \mathrm{CH}$ & 7.24 \\
9 & 128.5 & $2 \mathrm{CH}$ & 7.19 \\
10 & 128.1 & $2 \mathrm{CH}$ & 7.20 \\
11 & 126.8 & $\mathrm{CH}$ & 7.15 \\
12 & 126.4 & $\mathrm{CH}$ & 7.20 \\
13 & 124.4 & $\mathrm{CH}$ & 6.57 \\
14 & 112.8 & $\mathrm{CH}$ & 3.71 \\
15 & 55.3 & $\mathrm{CH}$ & 4.39 \\
16 & 47.1 & $\mathrm{CH}_{3}$ & 3.54 \\
17 & 34.4 & $\mathrm{CH}_{2}$ & 3.30 \\
18 & 26.8 & $\mathrm{CH}$ & $1.22,1.20$ \\
19 & 22.9 & $2 \mathrm{CH}_{3}$ & 2.12 \\
20 & 19.7 & $\mathrm{CH}_{3}$ & \\
\hline
\end{tabular}

Table 3. The Data Summarized from the HMBC Spectrum of 3ae

\begin{tabular}{cccc} 
entry & $\delta_{\mathrm{C}} / \mathrm{ppm}$ & $\begin{array}{c}\mathrm{H} \text { atoms with a long-range } \\
\text { coupling, } \delta_{\mathrm{H}} / \mathrm{ppm}\end{array}$ & $\begin{array}{c}\mathrm{H} \text { atoms with the }{ }^{1} J \\
\text { coupling, } \delta_{\mathrm{H}} / \mathrm{ppm}\end{array}$ \\
\hline 1 & 155.1 & $7.20,6.57,3.71,3.30$ & \\
2 & 143.8 & $7.22,7.15,4.39,3.54$ & \\
3 & $134.4,134.2$ & $7.20,6.57,4.39,3.30,2.12,1.22,1.21$ & \\
4 & $132.9,132.8$ & $7.20,6.57,4.39,3.54,2.12$ & 7.44 \\
5 & 131.1 & $7.44,7.20,3.54$ & \\
6 & 129.0 & 7.44 & 7.22 \\
7 & 128.5 & & \\
8 & 128.1 & $7.15,4.39$ & \\
9 & 126.8 & 7.44 & 3.71 \\
10 & 126.4 & 7.19 & \\
11 & 124.4 & 2.12 & \\
12 & 112.8 & & \\
13 & 55.3 & $7.20,(6.57), 3.54$ & \\
14 & 47.1 & 4.39 & 2.12 \\
15 & 34.4 & $7.20,6.57,1.22,1,20$ & \\
16 & 26.8 & 3.30 & \\
17 & 22.9 & 6.57 & \\
18 & 19.7 & & \\
\hline
\end{tabular}

The structure of compound 3ae deduced from the ${ }^{1} \mathrm{H}^{-1} \mathrm{H}$ COSY, HSQC, HMBC, and ROESY Spectra 
According to the HMBC spectrum of 3ae, the correlations of $\delta \mathrm{C}(134.2,132.9,47.1)$ and $\delta_{\mathrm{H}}(6.57)$, the correlation of $\delta_{\mathrm{C}}(124.4)$ and $\delta_{\mathrm{H}}(4.39,3.30)$, and the correlation of $\delta_{\mathrm{C}}(47.1)$ and $\delta_{\mathrm{H}}(7.20,6.57)$, and the ROESY spectrum of 3ae, the NOE effect between $\delta_{\mathrm{H}}$ (4.39) and $\delta_{\mathrm{H}}(7.20,2.12)$, together with the ${ }^{1} \mathrm{H}$ NMR, ${ }^{13} \mathrm{C}$ NMR, and HSQC spectra, the following structure of compound 3ae can be deduced (Figure 1).



Figure 1. The long-range coupling correlations of carbon atoms $\left(\delta_{\mathrm{C}} 132.9,124.4,47.1\right)$ and hydrogen atoms $\left(\delta_{\mathrm{H}}\right.$ $7.20,6.57,4.39,3.30)$ and the NOE effect between hydrogen atoms $(\delta \mathrm{H} 4.39$ and $\delta \mathrm{H} 7.20,2.12)$ in compound 3ae

\section{NOE correlation and relative configuration analysis of $( \pm)-3 \mathrm{ha}$ and $( \pm)-3 \mathrm{ia}$}

The products ( \pm )-3ha and ( \pm )-3ia were synthesized respectively from $(E)$ - $\beta$-methylstyphene $(\mathbf{2 h})$ and $(Z)$ - $\beta$-methylstyphene (2i). The selenoxide syn-elimination of the compounds ( \pm )-3ha and ( \pm )-3ia afforded compounds 5ha and 5ia. According to the mechanism of selenoxide elimination reaction, if a $\beta$-hydrogen atom is present, syn-elimination of selenoxide occurs easily to give the corresponding alkene (Scheme 1). ${ }^{[5]}$ Furthermore, according to the ROESY spectrum of 5ha, the correlation of $\delta_{\mathrm{H}}(7.24)$ and $\delta_{\mathrm{H}}(6.34)$, together with the ${ }^{1} \mathrm{H}$ NMR, and ${ }^{13} \mathrm{C}$ NMR spectra, the following structure of compound 5 ha can be deduced as (Z)-1-(1-phenyl-1-propenyl)-2,4,6-trimethoxybenzene. Therefore, the alkene 5ha was synthesized from (E)- $\beta$-methylstyphene (2h) via anti-addition with NPSP and syn-elimination of selenoxide (Scheme 2), whereas the alkene 5ia was obtained from ( $Z$ )- $\beta$-methylstyphene (2i) via anti-addition with NPSP and syn-elimination of selenoxide because of no correlation of $\delta_{\mathrm{H}}(7.25)$ and $\delta_{\mathrm{H}}(5.69)$ in the ROESY spectrum of 5ia (Scheme 3). Therefore, the $\mathrm{F}-\mathrm{C}$ alkylation reaction of $(E)-\beta$-methylstyphene (2h) produces $(1 S, 2 S)$ and $(1 R, 2 R)$ adducts $( \pm)$-3ha, and the reaction of $(Z)-\beta$-methylstyphene $(\mathbf{2 i})$ produces $(1 S, 2 R)$ and $(1 R, 2 S)$ adducts ( \pm )-3ia (Scheme 2 and 3)

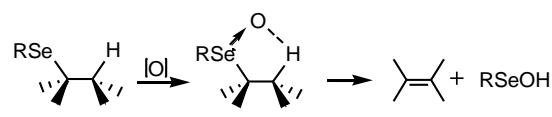

Scheme 1. The Mechanism of Selenoxide Elimination Reaction 


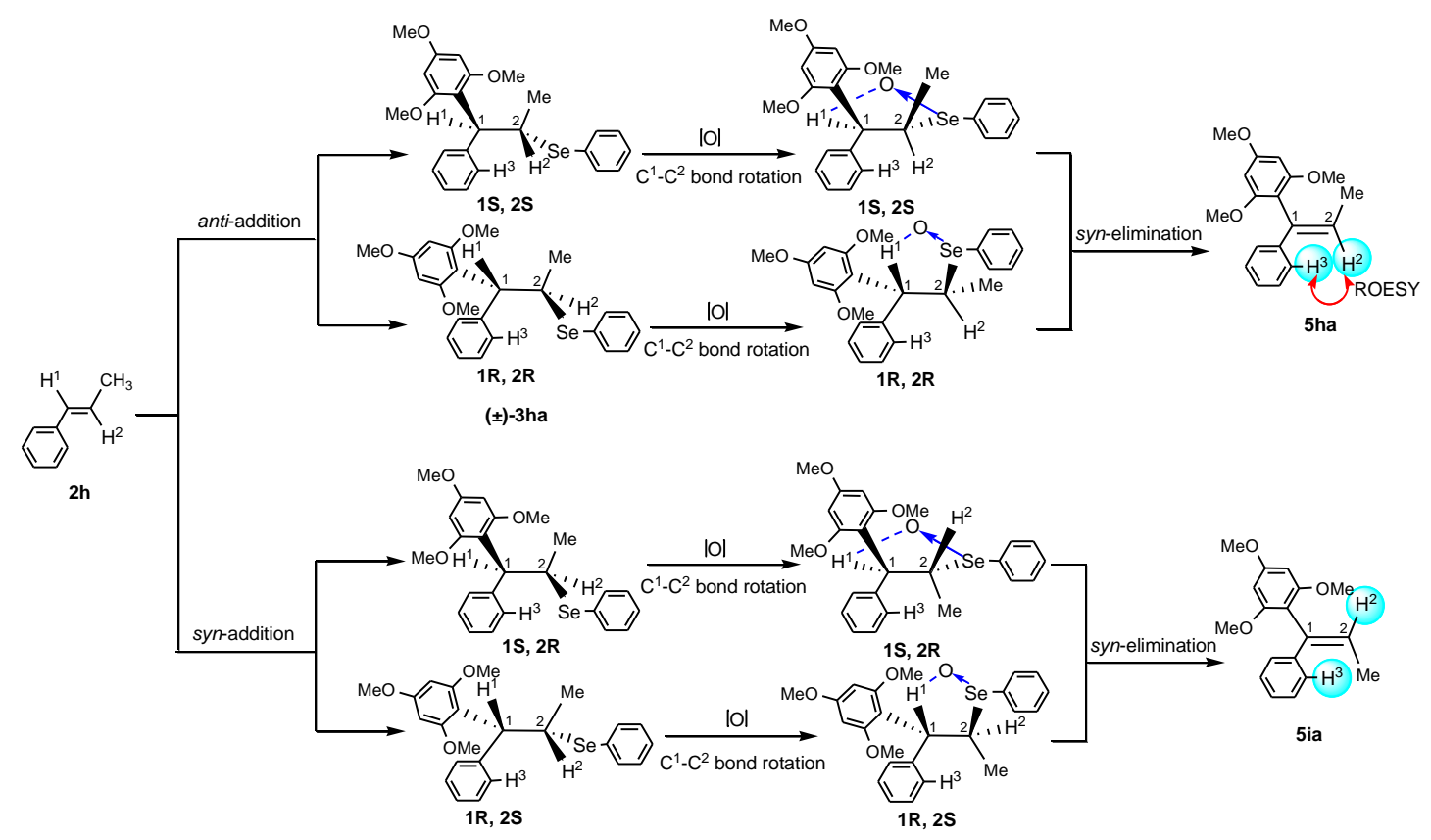

Scheme 2. Analysis of the Relative Configuration of the Product $( \pm)-3$ ha

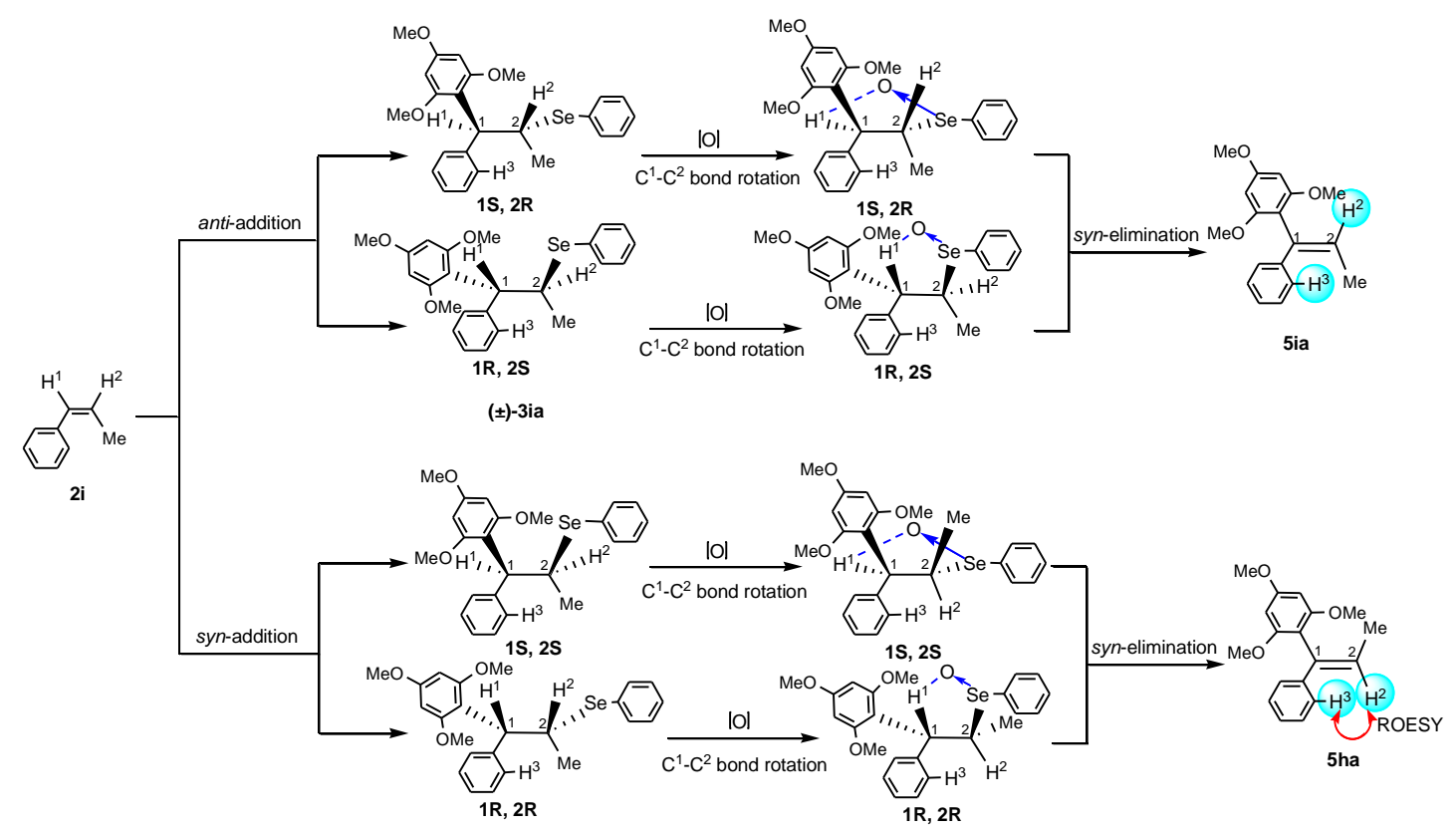

Scheme 3. Analysis of the Relative Configuration of the Product ( \pm )-3ia

\section{Byproducts of intermolecular selenium-promoted $\mathrm{F}-\mathrm{C}$ alkylation with styrene}

Table 4. Byproducts of Intermolecular Selenium-Promoted F-C Alkylation of Arenes with Styrene and NPSP ${ }^{a}$

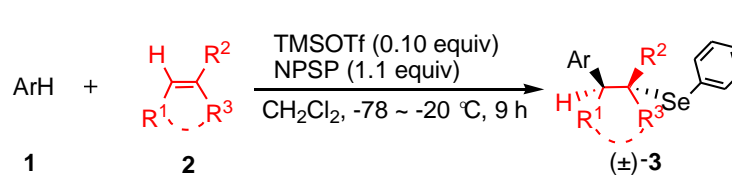<smiles>OC(C[Se]c1ccccc1)c1ccccc1</smiles>

16<smiles>CN1C(=O)c2ccccc2C1=O</smiles><smiles>[Al]CC([Se][Se]c1ccccc1)c1ccccc1</smiles>

17 
Entry ArH Ylkene Yield of $3^{a}(\%)$

3af, 65

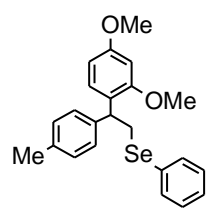

3ef, 66

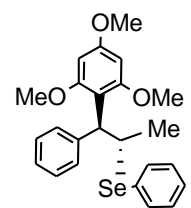

3ha, 58

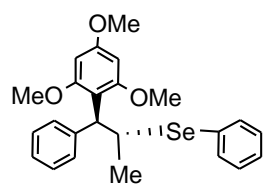

3ia, 65

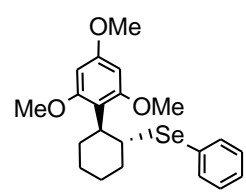

3ja, 67

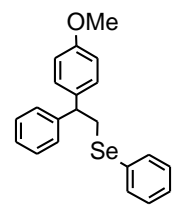

3aj, $<5$

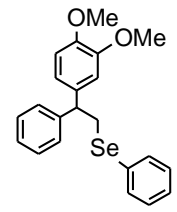

3ai, $<5$

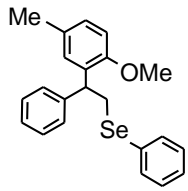

7<smiles>C=Cc1ccccc1</smiles>

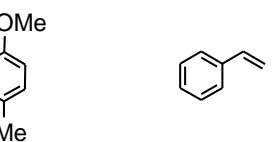

8

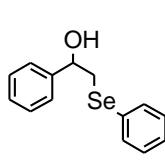

16 $^{[6]}, 45$

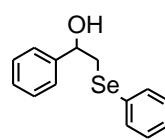

16, 50

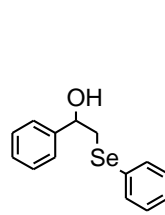

Yield of byproducts ${ }^{a}(\%)$

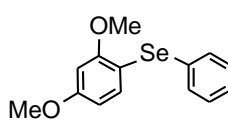

18f, 8

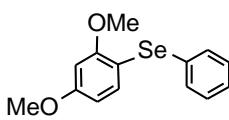

18f, 7

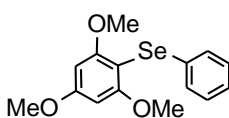

$18 \mathbf{a}^{[7]}, 10$

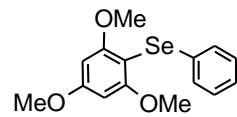

$18 \mathbf{a}^{[7]}, 8$

$18 \mathbf{a}^{[7]},<5$<smiles>COc1cc(OC)c([Se]c2ccccc2)c(OC)c1</smiles>

3al, $<5$

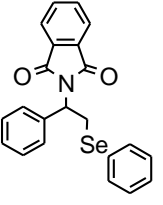

$17^{[6]}, 9$<smiles>COc1ccc([Se]c2ccccc2)cc1</smiles>

18j, 15<smiles>O=C1NC(=O)c2ccccc21</smiles><smiles>CC(C)CC(C)c1ccccc1</smiles>

$17,<5$

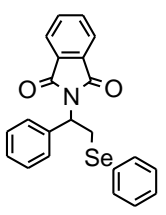

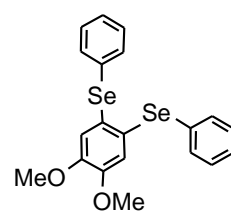

18i, 12

${ }^{a}$ Isolated yield.

A $\mathrm{TiCl}_{4}$-catalyzed intermolecular amidoselenenylation reaction of alkenes with NPSP was reported by us. ${ }^{[6]}$ The examples in Table 4 showed that besides the hydroxyselenenylated products and diaryl selenides, a 
small quantity of amidoselenenylated compounds generated in the intermolecular reaction of alkenes with ortho- and para-disubstituted benzenes, monosubstituted benzenes and NPSP (Table 4, entries 6-8). However, no amidoselenenylated compounds were observed even in those $\mathrm{F}-\mathrm{C}$ alkylation reactions which afforded the alkylated products such as 3af, 3ef, and $\mathbf{3 h}-\mathbf{j a}$ in the yields of 58-67\% (Table 4, entries 1-5). Therefore, it can be suggested that there is competition between the carbon-centered nucleophiles, water, and phthalimide counteranion in the reaction with episelenonium ion. A decrease in the nucleophilicity and the concentration of arene nucleophiles leads to an increase in the yield of the hydroxyselenenylation product and amidoselenenylation product.

\section{Characterization of compounds 18f, 18j, 18i, and $18 \mathrm{I}$}

\section{1,5-Dimethoxy-2-(phenylseleno)benzene (18f)}

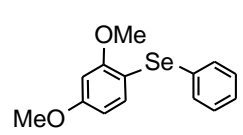

Yellow viscous liquid.

${ }^{1}$ H NMR $\left(300 \mathrm{MHz}, \mathrm{CDCl}_{3}\right): \delta 7.42-7.45(\mathrm{~m}, 2 \mathrm{H}), 7.22-7.29(\mathrm{~m}, 4 \mathrm{H}), 6.53(\mathrm{~d}, J=$ $2.4 \mathrm{~Hz}, 1 \mathrm{H}), 6.45(\mathrm{dd}, J=2.4,8.4 \mathrm{~Hz}, 1 \mathrm{H}), 3.85(\mathrm{~s}, 6 \mathrm{H})$.

${ }^{13}$ C NMR (75 MHz, $\left.\mathrm{CDCl}_{3}\right): \delta 161.3,159.2,135.3,132.4,131.1,129.1,126.8$, 110.4, 105.7, 99.0, 56.0, 55.5.

IR $\left(\mathrm{KBr}, \mathrm{cm}^{-1}\right): v$ 3060, 2946, 2838, 1583, 1469, 1398, 1295, 1203, 1025, 922, 830, 737, 690 .

HRMS (TOF): calcd for $\left[\mathrm{C}_{14} \mathrm{H}_{14} \mathrm{KNaO}_{2} \mathrm{Se}\right]: 355.9694$, found 355.9699 .

1-Methoxy-4-(phenylseleno)benzene (18j)

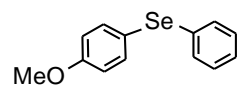

Yellow viscous liquid.

${ }^{1}$ H NMR $\left(300 \mathrm{MHz}, \mathrm{CDCl}_{3}\right): \delta 7.49(\mathrm{~d}, J=8.7 \mathrm{~Hz}, 2 \mathrm{H}), 7.31-7.30(\mathrm{~m}, 2 \mathrm{H})$, 7.15-7.22 (m, 3H), $6.83(\mathrm{~d}, J=8.7 \mathrm{~Hz}, 2 \mathrm{H}), 3.76$ (s, 3H).

${ }^{13}$ C NMR (75 MHz, $\left.\mathrm{CDCl}_{3}\right): \delta 160.0,136.6,133.3,131.0,129.2,126.5,120.0$, 115.2, 55.3.

IR $\left(\mathrm{KBr}, \mathrm{cm}^{-1}\right): v$ 3059, 2938, 1582, 1480, 1398, 1287, 1244, 1175, 1025, 819, 735, 687.

HRMS (TOF): calcd for $\left[\mathrm{C}_{13} \mathrm{H}_{13} \mathrm{OSe}\right]^{+}:$265.0132, found 265.0135 .

\section{1,2-Dimethoxy-4,5-bis(phenylseleno)benzene (18i)}

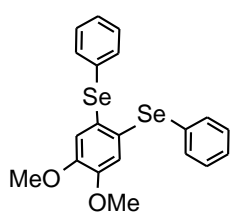

Yellow viscous liquid.

${ }^{1}$ H NMR $\left(300 \mathrm{MHz}, \mathrm{CDCl}_{3}\right): \delta 7.45-7.48(\mathrm{~m}, 4 \mathrm{H}), 7.27-7.31(\mathrm{~m}, 6 \mathrm{H}), 6.81(\mathrm{~s}, 2 \mathrm{H})$, $3.67(\mathrm{~s}, 6 \mathrm{H})$.

${ }^{13}$ C NMR (75 MHz, $\left.\mathrm{CDCl}_{3}\right): \delta 149.2,132.9,131.8,129.4,127.5,126.7,116.8,55.9$.

IR $\left(\mathrm{KBr}, \mathrm{cm}^{-1}\right): v$ 3052, 2930, 2839, 1569, 1483, 1438, 1341, 1251, 1199, 1023, 860, $741,691$.

HRMS (TOF): calcd for $\left[\mathrm{C}_{20} \mathrm{H}_{18} \mathrm{NaO}_{2} \mathrm{Se}_{2}\right]^{+}:$472.9535, found 472.9525 .

\section{1-Methyl-4-methoxy-3-(phenylseleno)benzene (18l)}<smiles>COc1ccc(C)cc1Oc1ccccc1</smiles>

Yellow viscous liquid.

${ }^{1}$ H NMR $\left(300 \mathrm{MHz}, \mathrm{CDCl}_{3}\right): \delta 7.53-7.55(\mathrm{~m}, 2 \mathrm{H}), 7.21-7.36(\mathrm{~m}, 3 \mathrm{H}), 6.85(\mathrm{dd}, J=$ $8.1,11.1 \mathrm{~Hz}, 2 \mathrm{H}), 6.84(\mathrm{~s}, 1 \mathrm{H}), 3.81$ (s, 3H), 2.14 (s, 3H);

${ }^{13}$ C NMR (75 MHz, $\left.\mathrm{CDCl}_{3}\right): \delta 155.1,134.8,132.3,129.4,129.0,128.5,127.8$, 121.1, 116.1, 110.6, 56.1, 20.5;

IR $\left(\mathrm{KBr}, \mathrm{cm}^{-1}\right): v$ 3057, 2939, 2840, 1582, 1475, 1384, 1346, 1244, 1186, 1059, 867, $805,743,687$. 


\section{ReactIRTM in situ FTIR analysis of the $\mathrm{F}-\mathrm{C}$ alkylation reaction}

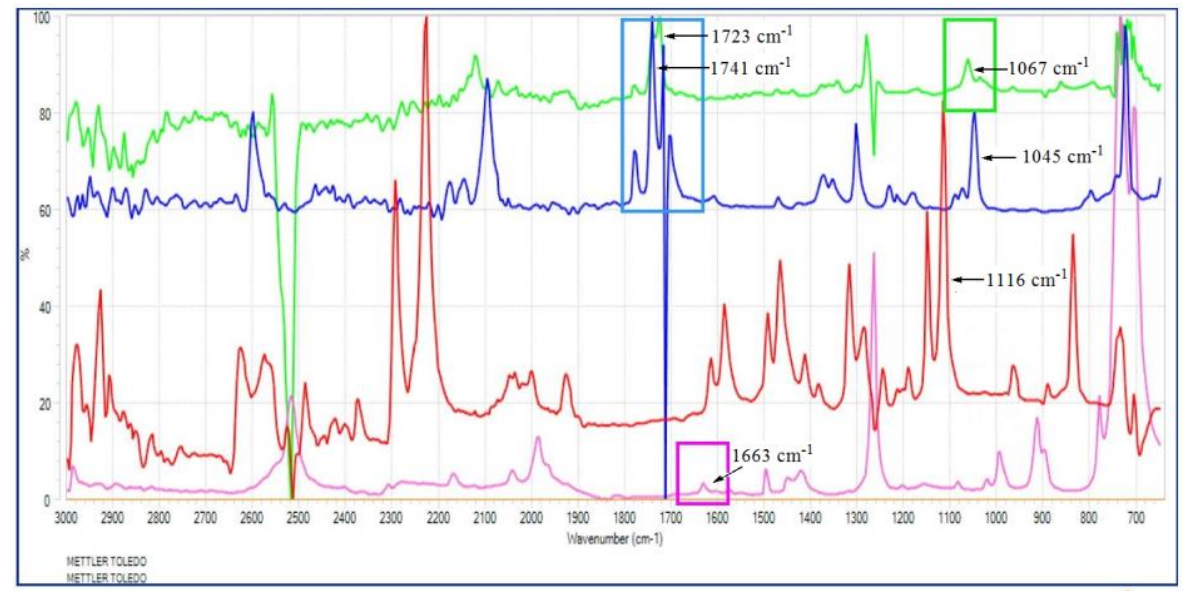

Figure 2. FTIR spectra of compounds $\mathbf{1 b}, \mathbf{2 a}, \mathbf{1 5}$, and NPSP

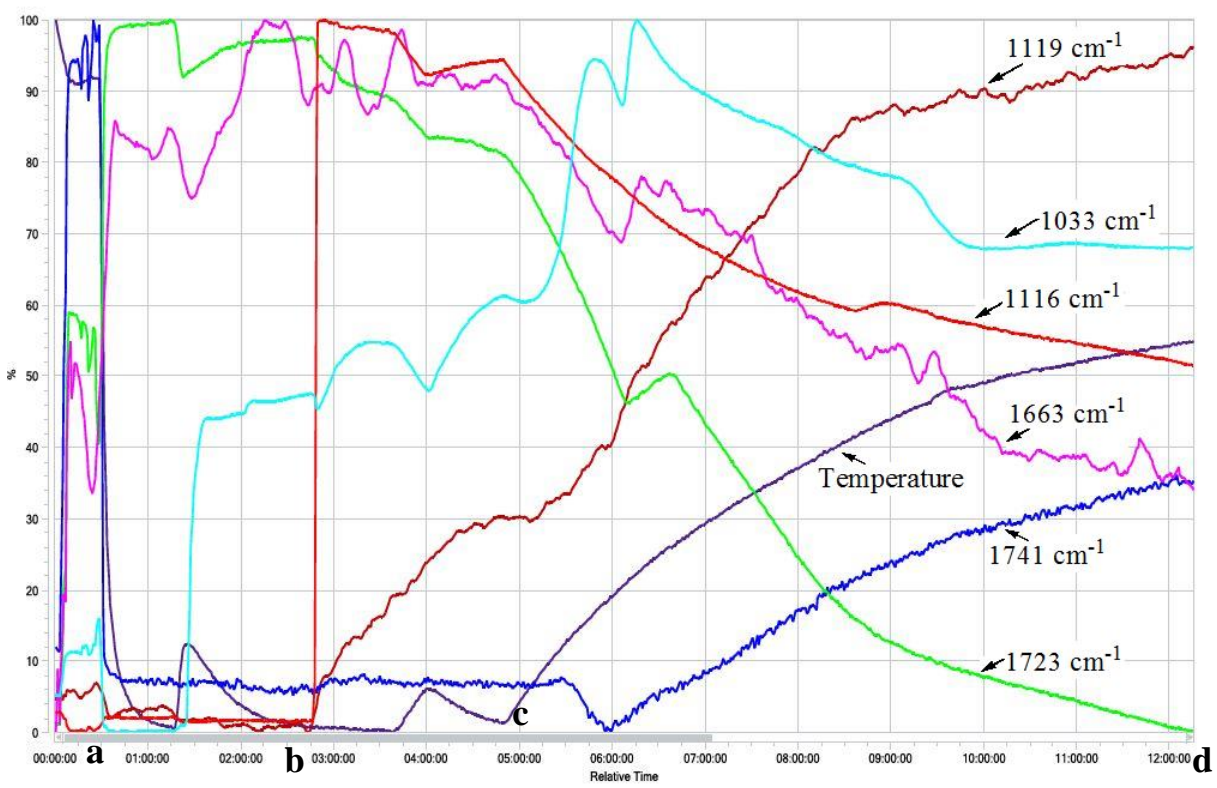

Point a: To a stirred solution of NPSP $(665 \mathrm{mg}, 2.2 \mathrm{mmol})$ in $\mathrm{CH}_{2} \mathrm{Cl}_{2}(20 \mathrm{~mL})$ at $-78{ }^{\circ} \mathrm{C}$, TMSOTf $(36.2 \mu \mathrm{L}$, $0.2 \mathrm{mmol}$ ) and styrene (208 $\mathrm{mg}, 2.0 \mathrm{mmol}$ ) was added.

Point b: To the stirred reaction mixture at $-78{ }^{\circ} \mathrm{C}$, 1-methoxy-2,3,5-trimethylbenzene $(\mathbf{1 b}, 300 \mathrm{mg}, 2.0$ mmol) was added.

Point c: The reaction mixture was allowed to slowly warm to $-20{ }^{\circ} \mathrm{C}$ after stirring for $2 \mathrm{~h}$ at $-78{ }^{\circ} \mathrm{C}$.

Point d: The reaction completed after the reaction mixture was allowed to slowly warm to $-20^{\circ} \mathrm{C}$ for $7 \mathrm{~h}$. 

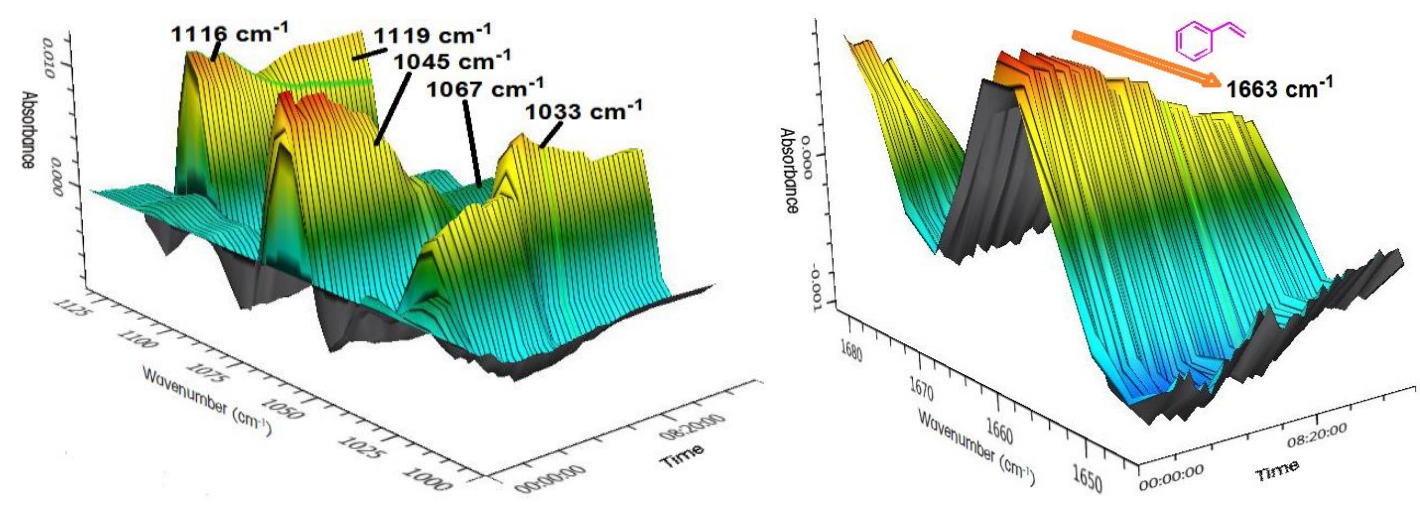

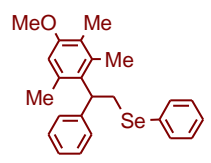

3ab

$v_{\mathrm{C}-\mathrm{O}-\mathrm{c}^{1119} \mathrm{~cm}^{-1}}$

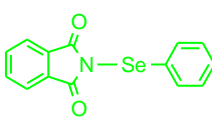

NPSP

$1045 \mathrm{~cm}^{-1}$ $v_{\mathrm{C}=0} 1723 \mathrm{~cm}^{-1}$

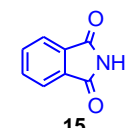

$1067 \mathrm{~cm}^{-1}$ $v_{\mathrm{C}=0} 1741 \mathrm{~cm}^{-1}$

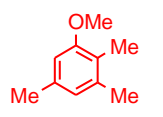

$1 b$

$v_{\mathrm{C}-\mathrm{O}-\mathrm{C}^{1116} \mathrm{~cm}^{-1}} \quad \begin{array}{ccc}2 \mathrm{a} & 13 \\ \mathrm{C}_{\mathrm{C}} \mathrm{C}^{1663 \mathrm{~cm}^{-1}} & v_{\mathrm{Si}-\mathrm{O}} 1033 \mathrm{~cm}^{-1}\end{array}$

Figure 3. Reaction profile and the resulting three-dimensional stack plot of the IR spectra collected every 1 min during the $\mathrm{F}-\mathrm{C}$ alkylation reaction of 1-methoxy-2,3,5-trimethylbenzene (1b) with styrene and NPSP in the presence of TMSOTf.

Deconvolution was performed by ReactIR ic 4.3 software; the absorption bands at 1119 and $1116 \mathrm{~cm}^{-1}$ were assigned to $\mathrm{C}-\mathrm{O}-\mathrm{C}$ bond vabritions of $\mathbf{3 a b}$ and $\mathbf{1 b}$ respectively; the absorption bands at 1723 and $1741 \mathrm{~cm}^{-1}$ were assigned to the carbonyl group vabritions of NPSP and 15 respectively; the absorption band at 1663 $\mathrm{cm}^{-1}$ was assigned to the carbon-carbon double bond vabrition of styrene; the absorption band at $1033 \mathrm{~cm}^{-1}$ was assigned to the oxygen-silicon bond vabrition of intermediate 13.

From Figure 2, NPSP reacted with TMSOTf to afford the silyl enol ether intermediate 13. The concentration of compound $\mathbf{1 3}$ increased with addition of styphene and decreased after the temperature of the reaction increased for about $1 \mathrm{~h}$ (see the absorption band at $1033 \mathrm{~cm}^{-1}$ ); Byproduct $\mathbf{1 5}$ formed after the temperature of the reaction increased for about $1 \mathrm{~h}$ (see the absorption band at $1067 \mathrm{~cm}^{-1}$ ). The ReactIR spectra revealed a plausible mechanism for the $\mathrm{F}-\mathrm{C}$ alkylation reaction.

\section{X-ray structure of compound 3ja}

Table 5. Crystal Data and Structure Refinement for $\mathbf{3 j a}$

Identification code

Empirical formula

Formula weight

Temperature

Wavelength

Crystal system, space group

Unit cell dimensions

Volume

Z, Calculated density
$3 \mathbf{j a}$

$\mathrm{C}_{21} \mathrm{H}_{26} \mathrm{O}_{3} \mathrm{Se}$

405.38

293(2) K

$0.71073 \mathrm{~A}$

Monoclinic, P2(1)/c

$$
\begin{array}{ll}
\mathrm{a}=7.4940(8) \AA & \alpha=90^{\circ} . \\
\mathrm{b}=15.0837(17) \AA & \beta=100.8840(10)^{\circ} . \\
\mathrm{c}=17.3328(19) \AA & \gamma=90^{\circ} .
\end{array}
$$

$1924.0(4) \mathrm{A}^{3}$

4, $1.399 \mathrm{Mg} / \mathrm{m}^{3}$ 


$\begin{array}{ll}\text { Absorption coefficient } & 1.968 \mathrm{~mm}^{-1} \\ \mathrm{~F}(000) & 840 \\ \text { Crystal size } & 0.30 \times 0.26 \times 0.24 \mathrm{~mm} \\ \theta \text { range for data collection } & 1.80 \text { to } 25.15^{\circ} . \\ \text { Limiting indices } & -8<=\mathrm{h}<=8,-18<=\mathrm{k}<=18,-17<=\mathrm{l}<=20 \\ \text { Reflections collected / unique } & 10788 / 3432[\mathrm{R}(\mathrm{int})=0.0317] \\ \text { Completeness to } \theta=25.15 & 99.9 \% \\ \text { Max. and min. transmission } & 0.6495 \text { and } 0.5898 \\ \text { Refinement method } & \text { Full-matrix least-squares on } \mathrm{F}^{2} \\ \text { Data / restraints / parameters } & 3432 / 0 / 229 \\ \text { Goodness-of-fit on } \mathrm{F}^{2} & 1.065 \\ \text { Final R indices [I }>2 \sigma(\mathrm{I})] & \mathrm{R} 1=0.0331, \mathrm{wR} 2=0.0788 \\ \text { R indices (all data) } & \mathrm{R} 1=0.0519, \mathrm{wR} 2=0.0874 \\ \text { Largest diff. peak and hole } & 0.227 \text { and }-0.388 \text { e. } \AA^{-3}\end{array}$

Table 6. Atomic Coordinates ( x 10 ${ }^{4}$ ) and Equivalent Isotropic Displacement Parameters $\left(\mathrm{A}^{2} \times 10^{3}\right)$ for $\mathbf{3 j a}$

\begin{tabular}{|c|c|c|c|c|}
\hline & $\mathrm{x}$ & $\mathrm{y}$ & $\mathrm{z}$ & $\mathrm{U}(\mathrm{eq})$ \\
\hline $\mathrm{O}(1)$ & 2601(3) & $10623(1)$ & $3377(1)$ & $49(1)$ \\
\hline $\mathrm{O}(2)$ & 1834(3) & $8890(1)$ & $5650(1)$ & $56(1)$ \\
\hline $\mathrm{O}(3)$ & $331(2)$ & $7722(1)$ & $3063(1)$ & $46(1)$ \\
\hline $\operatorname{Se}(1)$ & $5082(1)$ & $8513(1)$ & $2456(1)$ & $54(1)$ \\
\hline $\mathrm{C}(1)$ & $2166(3)$ & $9858(2)$ & $3731(1)$ & $35(1)$ \\
\hline $\mathrm{C}(2)$ & 2313(3) & $9796(2)$ & $4546(1)$ & $40(1)$ \\
\hline $\mathrm{C}(3)$ & $1770(3)$ & $9024(2)$ & $4859(1)$ & $41(1)$ \\
\hline $\mathrm{C}(4)$ & $1100(3)$ & $8322(2)$ & $4377(2)$ & $40(1)$ \\
\hline$C(5)$ & $1005(3)$ & 8391(1) & $3576(1)$ & $35(1)$ \\
\hline$C(6)$ & $1563(3)$ & $9155(1)$ & $3225(1)$ & $32(1)$ \\
\hline$C(7)$ & $1438(3)$ & $9245(2)$ & 2343(1) & $34(1)$ \\
\hline$C(8)$ & $-532(4)$ & $9310(2)$ & 1909(2) & $47(1)$ \\
\hline $\mathrm{C}(9)$ & $-684(5)$ & $9449(2)$ & 1031(2) & $62(1)$ \\
\hline$C(10)$ & $313(5)$ & $8726(2)$ & $683(2)$ & $61(1)$ \\
\hline $\mathrm{C}(11)$ & $2285(4)$ & $8672(2)$ & 1087(2) & $54(1)$ \\
\hline$C(12)$ & $2466(3)$ & $8531(2)$ & 1968(1) & $38(1)$ \\
\hline$C(13)$ & 2394(4) & $9618(2)$ & $6166(2)$ & $59(1)$ \\
\hline$C(14)$ & $2864(5)$ & $11413(2)$ & $3836(2)$ & $64(1)$ \\
\hline$C(15)$ & $-5(4)$ & 6881(2) & $3373(2)$ & $51(1)$ \\
\hline$C(16)$ & $5109(3)$ & $7561(2)$ & $3194(2)$ & $44(1)$ \\
\hline$C(17)$ & $5485(4)$ & $7714(2)$ & 3997(2) & $58(1)$ \\
\hline$C(18)$ & $5557(5)$ & $7021(2)$ & $4512(2)$ & $71(1)$ \\
\hline$C(19)$ & $5246(5)$ & $6171(2)$ & $4242(2)$ & $71(1)$ \\
\hline$C(20)$ & 4858(4) & $6010(2)$ & $3447(2)$ & $65(1)$ \\
\hline$C(21)$ & $4805(4)$ & $6700(2)$ & $2924(2)$ & $52(1)$ \\
\hline
\end{tabular}


$\mathrm{U}(\mathrm{eq})$ is defined as one third of the trace of the orthogonalized Uij tensor.

Table 7. Bond Lengths $[\AA]$ and Angles $\left[{ }^{\circ}\right]$ for $\mathbf{3 j a}$

\begin{tabular}{|c|c|}
\hline $\mathrm{O}(1)-\mathrm{C}(1)$ & $1.374(3)$ \\
\hline $\mathrm{O}(1)-\mathrm{C}(14)$ & $1.426(3)$ \\
\hline $\mathrm{O}(2)-\mathrm{C}(3)$ & $1.379(3)$ \\
\hline $\mathrm{O}(2)-\mathrm{C}(13)$ & $1.428(3)$ \\
\hline $\mathrm{O}(3)-\mathrm{C}(5)$ & $1.377(3)$ \\
\hline $\mathrm{O}(3)-\mathrm{C}(15)$ & $1.418(3)$ \\
\hline $\operatorname{Se}(1)-C(16)$ & $1.919(3)$ \\
\hline $\operatorname{Se}(1)-C(12)$ & $1.984(3)$ \\
\hline$C(1)-C(6)$ & $1.396(3)$ \\
\hline$C(1)-C(2)$ & $1.400(3)$ \\
\hline$C(2)-C(3)$ & $1.378(3)$ \\
\hline $\mathrm{C}(2)-\mathrm{H}(2)$ & 0.9300 \\
\hline$C(3)-C(4)$ & $1.383(3)$ \\
\hline$C(4)-C(5)$ & $1.381(3)$ \\
\hline $\mathrm{C}(4)-\mathrm{H}(4)$ & 0.9300 \\
\hline$C(5)-C(6)$ & $1.403(3)$ \\
\hline$C(6)-C(7)$ & $1.520(3)$ \\
\hline$C(7)-C(8)$ & $1.528(3)$ \\
\hline$C(7)-C(12)$ & $1.538(3)$ \\
\hline $\mathrm{C}(7)-\mathrm{H}(7)$ & 0.9800 \\
\hline $\mathrm{C}(8)-\mathrm{C}(9)$ & $1.519(4)$ \\
\hline $\mathrm{C}(8)-\mathrm{H}(8 \mathrm{~A})$ & 0.9700 \\
\hline $\mathrm{C}(8)-\mathrm{H}(8 \mathrm{~B})$ & 0.9700 \\
\hline $\mathrm{C}(9)-\mathrm{C}(10)$ & $1.511(4)$ \\
\hline $\mathrm{C}(9)-\mathrm{H}(9 \mathrm{~A})$ & 0.9700 \\
\hline $\mathrm{C}(9)-\mathrm{H}(9 \mathrm{~B})$ & 0.9700 \\
\hline $\mathrm{C}(10)-\mathrm{C}(11)$ & $1.513(4)$ \\
\hline $\mathrm{C}(10)-\mathrm{H}(10 \mathrm{~A})$ & 0.9700 \\
\hline $\mathrm{C}(10)-\mathrm{H}(10 \mathrm{~B})$ & 0.9700 \\
\hline $\mathrm{C}(11)-\mathrm{C}(12)$ & $1.523(3)$ \\
\hline $\mathrm{C}(11)-\mathrm{H}(11 \mathrm{~A})$ & 0.9700 \\
\hline $\mathrm{C}(11)-\mathrm{H}(11 \mathrm{~B})$ & 0.9700 \\
\hline $\mathrm{C}(12)-\mathrm{H}(12)$ & 0.9800 \\
\hline $\mathrm{C}(13)-\mathrm{H}(13 \mathrm{~A})$ & 0.9600 \\
\hline $\mathrm{C}(13)-\mathrm{H}(13 \mathrm{~B})$ & 0.9600 \\
\hline $\mathrm{C}(13)-\mathrm{H}(13 \mathrm{C})$ & 0.9600 \\
\hline $\mathrm{C}(14)-\mathrm{H}(14 \mathrm{~A})$ & 0.9600 \\
\hline $\mathrm{C}(14)-\mathrm{H}(14 \mathrm{~B})$ & 0.9600 \\
\hline $\mathrm{C}(14)-\mathrm{H}(14 \mathrm{C})$ & 0.9600 \\
\hline
\end{tabular}




\begin{tabular}{|c|c|}
\hline $\mathrm{C}(15)-\mathrm{H}(15 \mathrm{~A})$ & 0.9600 \\
\hline $\mathrm{C}(15)-\mathrm{H}(15 \mathrm{~B})$ & 0.9600 \\
\hline $\mathrm{C}(15)-\mathrm{H}(15 \mathrm{C})$ & 0.9600 \\
\hline$C(16)-C(21)$ & $1.384(4)$ \\
\hline$C(16)-C(17)$ & $1.387(4)$ \\
\hline $\mathrm{C}(17)-\mathrm{C}(18)$ & $1.368(4)$ \\
\hline $\mathrm{C}(17)-\mathrm{H}(17)$ & 0.9300 \\
\hline $\mathrm{C}(18)-\mathrm{C}(19)$ & $1.369(4)$ \\
\hline $\mathrm{C}(18)-\mathrm{H}(18)$ & 0.9300 \\
\hline$C(19)-C(20)$ & $1.375(5)$ \\
\hline $\mathrm{C}(19)-\mathrm{H}(19)$ & 0.9300 \\
\hline$C(20)-C(21)$ & $1.376(4)$ \\
\hline $\mathrm{C}(20)-\mathrm{H}(20)$ & 0.9300 \\
\hline $\mathrm{C}(21)-\mathrm{H}(21)$ & 0.9300 \\
\hline $\mathrm{C}(1)-\mathrm{O}(1)-\mathrm{C}(14)$ & 118.21(19) \\
\hline $\mathrm{C}(3)-\mathrm{O}(2)-\mathrm{C}(13)$ & $117.5(2)$ \\
\hline $\mathrm{C}(5)-\mathrm{O}(3)-\mathrm{C}(15)$ & $118.78(18)$ \\
\hline$C(16)-\operatorname{Se}(1)-C(12)$ & $100.34(10)$ \\
\hline $\mathrm{O}(1)-\mathrm{C}(1)-\mathrm{C}(6)$ & $115.6(2)$ \\
\hline $\mathrm{O}(1)-\mathrm{C}(1)-\mathrm{C}(2)$ & $121.8(2)$ \\
\hline$C(6)-C(1)-C(2)$ & $122.6(2)$ \\
\hline$C(3)-C(2)-C(1)$ & $118.7(2)$ \\
\hline $\mathrm{C}(3)-\mathrm{C}(2)-\mathrm{H}(2)$ & 120.6 \\
\hline $\mathrm{C}(1)-\mathrm{C}(2)-\mathrm{H}(2)$ & 120.6 \\
\hline $\mathrm{O}(2)-\mathrm{C}(3)-\mathrm{C}(2)$ & $123.9(2)$ \\
\hline $\mathrm{O}(2)-\mathrm{C}(3)-\mathrm{C}(4)$ & $115.4(2)$ \\
\hline$C(2)-C(3)-C(4)$ & $120.7(2)$ \\
\hline$C(5)-C(4)-C(3)$ & $119.5(2)$ \\
\hline $\mathrm{C}(5)-\mathrm{C}(4)-\mathrm{H}(4)$ & 120.2 \\
\hline $\mathrm{C}(3)-\mathrm{C}(4)-\mathrm{H}(4)$ & 120.2 \\
\hline $\mathrm{O}(3)-\mathrm{C}(5)-\mathrm{C}(4)$ & $122.4(2)$ \\
\hline $\mathrm{O}(3)-\mathrm{C}(5)-\mathrm{C}(6)$ & $115.2(2)$ \\
\hline$C(4)-C(5)-C(6)$ & $122.3(2)$ \\
\hline$C(1)-C(6)-C(5)$ & $116.0(2)$ \\
\hline$C(1)-C(6)-C(7)$ & $121.1(2)$ \\
\hline$C(5)-C(6)-C(7)$ & $122.8(2)$ \\
\hline$C(6)-C(7)-C(8)$ & $111.76(19)$ \\
\hline $\mathrm{C}(6)-\mathrm{C}(7)-\mathrm{C}(12)$ & $114.96(19)$ \\
\hline $\mathrm{C}(8)-\mathrm{C}(7)-\mathrm{C}(12)$ & $110.5(2)$ \\
\hline $\mathrm{C}(6)-\mathrm{C}(7)-\mathrm{H}(7)$ & 106.3 \\
\hline $\mathrm{C}(8)-\mathrm{C}(7)-\mathrm{H}(7)$ & 106.3 \\
\hline $\mathrm{C}(12)-\mathrm{C}(7)-\mathrm{H}(7)$ & 106.3 \\
\hline $\mathrm{C}(9)-\mathrm{C}(8)-\mathrm{C}(7)$ & $112.6(2)$ \\
\hline
\end{tabular}




\begin{tabular}{|c|c|}
\hline $\mathrm{C}(9)-\mathrm{C}(8)-\mathrm{H}(8 \mathrm{~A})$ & 109.1 \\
\hline $\mathrm{C}(7)-\mathrm{C}(8)-\mathrm{H}(8 \mathrm{~A})$ & 109.1 \\
\hline $\mathrm{C}(9)-\mathrm{C}(8)-\mathrm{H}(8 \mathrm{~B})$ & 109.1 \\
\hline $\mathrm{C}(7)-\mathrm{C}(8)-\mathrm{H}(8 \mathrm{~B})$ & 109.1 \\
\hline $\mathrm{H}(8 \mathrm{~A})-\mathrm{C}(8)-\mathrm{H}(8 \mathrm{~B})$ & 107.8 \\
\hline $\mathrm{C}(10)-\mathrm{C}(9)-\mathrm{C}(8)$ & $110.5(2)$ \\
\hline $\mathrm{C}(10)-\mathrm{C}(9)-\mathrm{H}(9 \mathrm{~A})$ & 109.5 \\
\hline $\mathrm{C}(8)-\mathrm{C}(9)-\mathrm{H}(9 \mathrm{~A})$ & 109.5 \\
\hline $\mathrm{C}(10)-\mathrm{C}(9)-\mathrm{H}(9 \mathrm{~B})$ & 109.5 \\
\hline $\mathrm{C}(8)-\mathrm{C}(9)-\mathrm{H}(9 \mathrm{~B})$ & 109.5 \\
\hline $\mathrm{H}(9 \mathrm{~A})-\mathrm{C}(9)-\mathrm{H}(9 \mathrm{~B})$ & 108.1 \\
\hline $\mathrm{C}(9)-\mathrm{C}(10)-\mathrm{C}(11)$ & $111.6(2)$ \\
\hline $\mathrm{C}(9)-\mathrm{C}(10)-\mathrm{H}(10 \mathrm{~A})$ & 109.3 \\
\hline $\mathrm{C}(11)-\mathrm{C}(10)-\mathrm{H}(10 \mathrm{~A})$ & 109.3 \\
\hline $\mathrm{C}(9)-\mathrm{C}(10)-\mathrm{H}(10 \mathrm{~B})$ & 109.3 \\
\hline $\mathrm{C}(11)-\mathrm{C}(10)-\mathrm{H}(10 \mathrm{~B})$ & 109.3 \\
\hline $\mathrm{H}(10 \mathrm{~A})-\mathrm{C}(10)-\mathrm{H}(10 \mathrm{~B})$ & 108.0 \\
\hline $\mathrm{C}(10)-\mathrm{C}(11)-\mathrm{C}(12)$ & $111.4(2)$ \\
\hline $\mathrm{C}(10)-\mathrm{C}(11)-\mathrm{H}(11 \mathrm{~A})$ & 109.3 \\
\hline $\mathrm{C}(12)-\mathrm{C}(11)-\mathrm{H}(11 \mathrm{~A})$ & 109.3 \\
\hline $\mathrm{C}(10)-\mathrm{C}(11)-\mathrm{H}(11 \mathrm{~B})$ & 109.3 \\
\hline $\mathrm{C}(12)-\mathrm{C}(11)-\mathrm{H}(11 \mathrm{~B})$ & 109.3 \\
\hline $\mathrm{H}(11 \mathrm{~A})-\mathrm{C}(11)-\mathrm{H}(11 \mathrm{~B})$ & 108.0 \\
\hline $\mathrm{C}(11)-\mathrm{C}(12)-\mathrm{C}(7)$ & 111.7(2) \\
\hline $\mathrm{C}(11)-\mathrm{C}(12)-\mathrm{Se}(1)$ & $108.87(17)$ \\
\hline $\mathrm{C}(7)-\mathrm{C}(12)-\mathrm{Se}(1)$ & $111.25(16)$ \\
\hline $\mathrm{C}(11)-\mathrm{C}(12)-\mathrm{H}(12)$ & 108.3 \\
\hline $\mathrm{C}(7)-\mathrm{C}(12)-\mathrm{H}(12)$ & 108.3 \\
\hline $\mathrm{Se}(1)-\mathrm{C}(12)-\mathrm{H}(12)$ & 108.3 \\
\hline $\mathrm{O}(2)-\mathrm{C}(13)-\mathrm{H}(13 \mathrm{~A})$ & 109.5 \\
\hline $\mathrm{O}(2)-\mathrm{C}(13)-\mathrm{H}(13 \mathrm{~B})$ & 109.5 \\
\hline $\mathrm{H}(13 \mathrm{~A})-\mathrm{C}(13)-\mathrm{H}(13 \mathrm{~B})$ & 109.5 \\
\hline $\mathrm{O}(2)-\mathrm{C}(13)-\mathrm{H}(13 \mathrm{C})$ & 109.5 \\
\hline $\mathrm{H}(13 \mathrm{~A})-\mathrm{C}(13)-\mathrm{H}(13 \mathrm{C})$ & 109.5 \\
\hline $\mathrm{H}(13 \mathrm{~B})-\mathrm{C}(13)-\mathrm{H}(13 \mathrm{C})$ & 109.5 \\
\hline $\mathrm{O}(1)-\mathrm{C}(14)-\mathrm{H}(14 \mathrm{~A})$ & 109.5 \\
\hline $\mathrm{O}(1)-\mathrm{C}(14)-\mathrm{H}(14 \mathrm{~B})$ & 109.5 \\
\hline $\mathrm{H}(14 \mathrm{~A})-\mathrm{C}(14)-\mathrm{H}(14 \mathrm{~B})$ & 109.5 \\
\hline $\mathrm{O}(1)-\mathrm{C}(14)-\mathrm{H}(14 \mathrm{C})$ & 109.5 \\
\hline $\mathrm{H}(14 \mathrm{~A})-\mathrm{C}(14)-\mathrm{H}(14 \mathrm{C})$ & 109.5 \\
\hline $\mathrm{H}(14 \mathrm{~B})-\mathrm{C}(14)-\mathrm{H}(14 \mathrm{C})$ & 109.5 \\
\hline $\mathrm{O}(3)-\mathrm{C}(15)-\mathrm{H}(15 \mathrm{~A})$ & 109.5 \\
\hline $\mathrm{O}(3)-\mathrm{C}(15)-\mathrm{H}(15 \mathrm{~B})$ & 109.5 \\
\hline $\mathrm{H}(15 \mathrm{~A})-\mathrm{C}(15)-\mathrm{H}(15 \mathrm{~B})$ & 109.5 \\
\hline
\end{tabular}




$\begin{array}{ll}\mathrm{O}(3)-\mathrm{C}(15)-\mathrm{H}(15 \mathrm{C}) & 109.5 \\ \mathrm{H}(15 \mathrm{~A})-\mathrm{C}(15)-\mathrm{H}(15 \mathrm{C}) & 109.5 \\ \mathrm{H}(15 \mathrm{~B})-\mathrm{C}(15)-\mathrm{H}(15 \mathrm{C}) & 109.5 \\ \mathrm{C}(21)-\mathrm{C}(16)-\mathrm{C}(17) & 118.9(3) \\ \mathrm{C}(21)-\mathrm{C}(16)-\mathrm{Se}(1) & 119.8(2) \\ \mathrm{C}(17)-\mathrm{C}(16)-\mathrm{Se}(1) & 121.3(2) \\ \mathrm{C}(18)-\mathrm{C}(17)-\mathrm{C}(16) & 120.3(3) \\ \mathrm{C}(18)-\mathrm{C}(17)-\mathrm{H}(17) & 119.9 \\ \mathrm{C}(16)-\mathrm{C}(17)-\mathrm{H}(17) & 119.9 \\ \mathrm{C}(19)-\mathrm{C}(18)-\mathrm{C}(17) & 120.6(3) \\ \mathrm{C}(19)-\mathrm{C}(18)-\mathrm{H}(18) & 119.7 \\ \mathrm{C}(17)-\mathrm{C}(18)-\mathrm{H}(18) & 119.7 \\ \mathrm{C}(18)-\mathrm{C}(19)-\mathrm{C}(20) & 119.8(3) \\ \mathrm{C}(18)-\mathrm{C}(19)-\mathrm{H}(19) & 120.1 \\ \mathrm{C}(20)-\mathrm{C}(19)-\mathrm{H}(19) & 120.1 \\ \mathrm{C}(19)-\mathrm{C}(20)-\mathrm{C}(21) & 120.0(3) \\ \mathrm{C}(19)-\mathrm{C}(20)-\mathrm{H}(20) & 120.0 \\ \mathrm{C}(21)-\mathrm{C}(20)-\mathrm{H}(20) & 120.0 \\ \mathrm{C}(20)-\mathrm{C}(21)-\mathrm{C}(16) & 120.4(3) \\ \mathrm{C}(20)-\mathrm{C}(21)-\mathrm{H}(21) & 119.8 \\ \mathrm{C}(16)-\mathrm{C}(21)-\mathrm{H}(21) & 119.8\end{array}$

Symmetry transformations used to generate equivalent atoms:

Table 8. Anisotropic Displacement Parameters $\left(\mathrm{A}^{2} \times 10^{3}\right)$ for $\mathbf{3 j a}$

$\begin{array}{lcccrrr} & \mathrm{U} 11 & \mathrm{U} 22 & \mathrm{U} 33 & \mathrm{U} 23 & \mathrm{U} 13 & \mathrm{U} 12 \\ \mathrm{O}(1) & 77(1) & 33(1) & 39(1) & -4(1) & 18(1) & -13(1) \\ \mathrm{O}(2) & 81(2) & 59(1) & 27(1) & 5(1) & 11(1) & 5(1) \\ \mathrm{O}(3) & 68(1) & 34(1) & 37(1) & -2(1) & 13(1) & -15(1) \\ \mathrm{Se}(1) & 42(1) & 53(1) & 69(1) & 0(1) & 20(1) & -5(1) \\ \mathrm{C}(1) & 38(1) & 34(1) & 34(1) & 1(1) & 9(1) & 0(1) \\ \mathrm{C}(2) & 47(2) & 42(1) & 32(2) & -6(1) & 5(1) & 0(1) \\ \mathrm{C}(3) & 44(2) & 49(2) & 29(1) & 2(1) & 9(1) & 9(1) \\ \mathrm{C}(4) & 48(2) & 38(1) & 36(2) & 7(1) & 10(1) & 1(1) \\ \mathrm{C}(5) & 41(1) & 33(1) & 31(1) & 0(1) & 8(1) & 0(1) \\ \mathrm{C}(6) & 37(1) & 32(1) & 28(1) & 0(1) & 9(1) & 1(1) \\ \mathrm{C}(7) & 43(2) & 29(1) & 31(1) & 0(1) & 8(1) & -3(1) \\ \mathrm{C}(8) & 51(2) & 47(2) & 39(2) & 0(1) & 3(1) & 6(1) \\ \mathrm{C}(9) & 88(2) & 55(2) & 36(2) & -2(1) & -7(2) & 11(2) \\ \mathrm{C}(10) & 96(3) & 53(2) & 30(2) & -5(1) & 3(2) & -3(2) \\ \mathrm{C}(11) & 82(2) & 46(2) & 41(2) & -9(1) & 27(2) & -9(2) \\ \mathrm{C}(12) & 45(2) & 35(1) & 35(1) & -2(1) & 13(1) & -5(1) \\ \mathrm{C}(13) & 66(2) & 80(2) & 29(2) & -5(2) & 3(1) & 19(2)\end{array}$




$\begin{array}{llllrrr}\mathrm{C}(14) & 99(3) & 37(2) & 61(2) & -12(1) & 29(2) & -12(2) \\ \mathrm{C}(15) & 63(2) & 34(1) & 56(2) & 2(1) & 13(2) & -9(1) \\ \mathrm{C}(16) & 33(1) & 45(2) & 53(2) & -8(1) & 7(1) & 4(1) \\ \mathrm{C}(17) & 62(2) & 47(2) & 60(2) & -13(2) & 1(2) & 2(1) \\ \mathrm{C}(18) & 88(3) & 69(2) & 50(2) & -3(2) & -6(2) & 4(2) \\ \mathrm{C}(19) & 67(2) & 59(2) & 81(3) & 17(2) & -4(2) & 7(2) \\ \mathrm{C}(20) & 61(2) & 41(2) & 88(3) & -7(2) & -3(2) & 1(1) \\ \mathrm{C}(21) & 50(2) & 50(2) & 55(2) & -14(1) & 3(1) & 1(1)\end{array}$

The anisotropic displacement factor exponent takes the form: $-2 \mathrm{pi}^{2}\left[\mathrm{~h}^{2} \mathrm{a}^{* 2} \mathrm{U} 11+\ldots+2 \mathrm{~h} \mathrm{k} \mathrm{a}^{*} \mathrm{~b}^{*}\right.$ U12 ]

Table 9. Hydrogen Coordinates ( x 10 ${ }^{4}$ ) and Isotropic Displacement Parameters $\left(\mathrm{A}^{2} \times 10^{3}\right)$ for $\mathbf{3 j a .}$

\begin{tabular}{|c|c|c|c|c|}
\hline & $\mathrm{x}$ & $\mathrm{y}$ & $\mathrm{Z}$ & $\mathrm{U}(\mathrm{eq})$ \\
\hline $\mathrm{H}(2)$ & 2769 & 10267 & 4871 & 48 \\
\hline $\mathrm{H}(4)$ & 717 & 7807 & 4591 & 49 \\
\hline $\mathrm{H}(7)$ & 2011 & 9812 & 2259 & 41 \\
\hline $\mathrm{H}(8 \mathrm{~A})$ & -1167 & 8770 & 1996 & 56 \\
\hline $\mathrm{H}(8 \mathrm{~B})$ & -1121 & 9799 & 2124 & 56 \\
\hline $\mathrm{H}(9 \mathrm{~A})$ & -1954 & 9448 & 778 & 75 \\
\hline $\mathrm{H}(9 \mathrm{~B})$ & -173 & 10021 & 937 & 75 \\
\hline $\mathrm{H}(10 \mathrm{~A})$ & 247 & 8840 & 128 & 73 \\
\hline $\mathrm{H}(10 \mathrm{~B})$ & -274 & 8161 & 734 & 73 \\
\hline $\mathrm{H}(11 \mathrm{~A})$ & 2901 & 9216 & 993 & 65 \\
\hline $\mathrm{H}(11 \mathrm{~B})$ & 2868 & 8186 & 864 & 65 \\
\hline $\mathrm{H}(12)$ & 1943 & 7952 & 2053 & 45 \\
\hline $\mathrm{H}(13 \mathrm{~A})$ & 3638 & 9761 & 6156 & 89 \\
\hline $\mathrm{H}(13 \mathrm{~B})$ & 2280 & 9459 & 6691 & 89 \\
\hline $\mathrm{H}(13 \mathrm{C})$ & 1641 & 10123 & 5998 & 89 \\
\hline $\mathrm{H}(14 \mathrm{~A})$ & 1826 & 11512 & 4076 & 96 \\
\hline $\mathrm{H}(14 \mathrm{~B})$ & 3010 & 11907 & 3504 & 96 \\
\hline $\mathrm{H}(14 \mathrm{C})$ & 3931 & 11354 & 4237 & 96 \\
\hline $\mathrm{H}(15 \mathrm{~A})$ & 1085 & 6668 & 3704 & 76 \\
\hline $\mathrm{H}(15 \mathrm{~B})$ & -377 & 6471 & 2950 & 76 \\
\hline $\mathrm{H}(15 \mathrm{C})$ & -949 & 6934 & 3675 & 76 \\
\hline $\mathrm{H}(17)$ & 5690 & 8288 & 4187 & 69 \\
\hline $\mathrm{H}(18)$ & 5820 & 7128 & 5050 & 86 \\
\hline $\mathrm{H}(19)$ & 5297 & 5704 & 4596 & 85 \\
\hline $\mathrm{H}(20)$ & 4631 & 5434 & 3262 & 79 \\
\hline $\mathrm{H}(21)$ & 4564 & 6588 & 2387 & 63 \\
\hline
\end{tabular}


Table 10. Torsion Angles $\left[^{\circ}\right]$ for $\mathbf{3 j a}$

\begin{tabular}{|c|c|}
\hline $\mathrm{C}(14)-\mathrm{O}(1)-\mathrm{C}(1)-\mathrm{C}(6)$ & $167.4(2)$ \\
\hline $\mathrm{C}(14)-\mathrm{O}(1)-\mathrm{C}(1)-\mathrm{C}(2)$ & $-12.3(3)$ \\
\hline $\mathrm{O}(1)-\mathrm{C}(1)-\mathrm{C}(2)-\mathrm{C}(3)$ & $176.8(2)$ \\
\hline$C(6)-C(1)-C(2)-C(3)$ & $-2.8(4)$ \\
\hline $\mathrm{C}(13)-\mathrm{O}(2)-\mathrm{C}(3)-\mathrm{C}(2)$ & $4.0(4)$ \\
\hline $\mathrm{C}(13)-\mathrm{O}(2)-\mathrm{C}(3)-\mathrm{C}(4)$ & $-175.7(2)$ \\
\hline $\mathrm{C}(1)-\mathrm{C}(2)-\mathrm{C}(3)-\mathrm{O}(2)$ & $-179.3(2)$ \\
\hline $\mathrm{C}(1)-\mathrm{C}(2)-\mathrm{C}(3)-\mathrm{C}(4)$ & $0.3(4)$ \\
\hline $\mathrm{O}(2)-\mathrm{C}(3)-\mathrm{C}(4)-\mathrm{C}(5)$ & $-179.2(2)$ \\
\hline $\mathrm{C}(2)-\mathrm{C}(3)-\mathrm{C}(4)-\mathrm{C}(5)$ & $1.1(4)$ \\
\hline $\mathrm{C}(15)-\mathrm{O}(3)-\mathrm{C}(5)-\mathrm{C}(4)$ & $-10.4(3)$ \\
\hline $\mathrm{C}(15)-\mathrm{O}(3)-\mathrm{C}(5)-\mathrm{C}(6)$ & $170.6(2)$ \\
\hline $\mathrm{C}(3)-\mathrm{C}(4)-\mathrm{C}(5)-\mathrm{O}(3)$ & $-179.1(2)$ \\
\hline$C(3)-C(4)-C(5)-C(6)$ & $-0.2(4)$ \\
\hline $\mathrm{O}(1)-\mathrm{C}(1)-\mathrm{C}(6)-\mathrm{C}(5)$ & $-176.1(2)$ \\
\hline$C(2)-C(1)-C(6)-C(5)$ & $3.6(3)$ \\
\hline $\mathrm{O}(1)-\mathrm{C}(1)-\mathrm{C}(6)-\mathrm{C}(7)$ & $0.9(3)$ \\
\hline$C(2)-C(1)-C(6)-C(7)$ & $-179.4(2)$ \\
\hline $\mathrm{O}(3)-\mathrm{C}(5)-\mathrm{C}(6)-\mathrm{C}(1)$ & $177.0(2)$ \\
\hline $\mathrm{C}(4)-\mathrm{C}(5)-\mathrm{C}(6)-\mathrm{C}(1)$ & $-2.1(4)$ \\
\hline $\mathrm{O}(3)-\mathrm{C}(5)-\mathrm{C}(6)-\mathrm{C}(7)$ & $0.0(3)$ \\
\hline$C(4)-C(5)-C(6)-C(7)$ & $-179.0(2)$ \\
\hline$C(1)-C(6)-C(7)-C(8)$ & $-107.4(3)$ \\
\hline $\mathrm{C}(5)-\mathrm{C}(6)-\mathrm{C}(7)-\mathrm{C}(8)$ & $69.4(3)$ \\
\hline $\mathrm{C}(1)-\mathrm{C}(6)-\mathrm{C}(7)-\mathrm{C}(12)$ & $125.5(2)$ \\
\hline$C(5)-C(6)-C(7)-C(12)$ & $-57.7(3)$ \\
\hline $\mathrm{C}(6)-\mathrm{C}(7)-\mathrm{C}(8)-\mathrm{C}(9)$ & $176.9(2)$ \\
\hline $\mathrm{C}(12)-\mathrm{C}(7)-\mathrm{C}(8)-\mathrm{C}(9)$ & $-53.7(3)$ \\
\hline $\mathrm{C}(7)-\mathrm{C}(8)-\mathrm{C}(9)-\mathrm{C}(10)$ & $55.6(3)$ \\
\hline $\mathrm{C}(8)-\mathrm{C}(9)-\mathrm{C}(10)-\mathrm{C}(11)$ & $-56.4(3)$ \\
\hline $\mathrm{C}(9)-\mathrm{C}(10)-\mathrm{C}(11)-\mathrm{C}(12)$ & $56.6(3)$ \\
\hline $\mathrm{C}(10)-\mathrm{C}(11)-\mathrm{C}(12)-\mathrm{C}(7)$ & $-54.7(3)$ \\
\hline $\mathrm{C}(10)-\mathrm{C}(11)-\mathrm{C}(12)-\mathrm{Se}(1)$ & $-177.89(17)$ \\
\hline$C(6)-C(7)-C(12)-C(11)$ & $-179.6(2)$ \\
\hline $\mathrm{C}(8)-\mathrm{C}(7)-\mathrm{C}(12)-\mathrm{C}(11)$ & $52.8(3)$ \\
\hline $\mathrm{C}(6)-\mathrm{C}(7)-\mathrm{C}(12)-\mathrm{Se}(1)$ & $-57.7(2)$ \\
\hline $\mathrm{C}(8)-\mathrm{C}(7)-\mathrm{C}(12)-\mathrm{Se}(1)$ & $174.62(16)$ \\
\hline $\mathrm{C}(16)-\mathrm{Se}(1)-\mathrm{C}(12)-\mathrm{C}(11)$ & $-138.97(17)$ \\
\hline $\mathrm{C}(16)-\mathrm{Se}(1)-\mathrm{C}(12)-\mathrm{C}(7)$ & $97.57(16)$ \\
\hline$C(12)-S e(1)-C(16)-C(21)$ & $68.9(2)$ \\
\hline $\mathrm{C}(12)-\mathrm{Se}(1)-\mathrm{C}(16)-\mathrm{C}(17)$ & $-113.1(2)$ \\
\hline $\mathrm{C}(21)-\mathrm{C}(16)-\mathrm{C}(17)-\mathrm{C}(18)$ & $0.1(4)$ \\
\hline $\operatorname{Se}(1)-C(16)-C(17)-C(18)$ & $-177.8(2)$ \\
\hline
\end{tabular}




$\begin{array}{lr}C(16)-C(17)-C(18)-C(19) & -0.5(5) \\ C(17)-C(18)-C(19)-C(20) & 0.0(5) \\ C(18)-C(19)-C(20)-C(21) & 0.8(5) \\ C(19)-C(20)-C(21)-C(16) & -1.2(5) \\ C(17)-C(16)-C(21)-C(20) & 0.7(4) \\ S e(1)-C(16)-C(21)-C(20) & 178.6(2)\end{array}$

Symmetry transformations used to generate equivalent atoms:

Table 11. Hydrogen Bonds for $\mathbf{3 j a}\left[\AA\right.$ and $^{\circ}$ ]

\begin{tabular}{lllll}
\hline D-H...A & d(D-H) & d(H...A $)$ & d(D...A $)$ & $<($ DHA $)$ \\
\hline
\end{tabular}

$\mathrm{X}$-ray structure of compound $\mathbf{3 j a}$

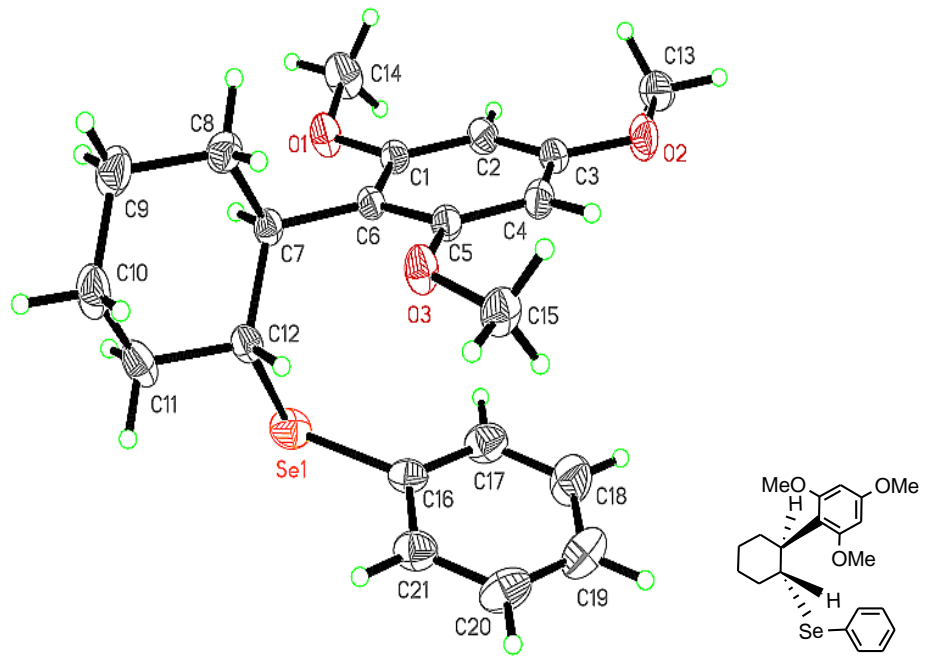

\section{X-ray structure of compound 5ab}

Table 12. Crystal Data and Structure Refinement for 5ab.

\begin{tabular}{lll}
\hline Identification code & $\mathbf{5 a b}$ & \\
Empirical formula & $\mathrm{C}_{18} \mathrm{H}_{20} \mathrm{O}$ & \\
Formula weight & 252.34 & \\
Temperature & $298(2) \mathrm{K}$ & \\
Wavelength & $0.71073 \AA$ & \\
Crystal system, space group & Triclinic, $\mathrm{P}-1$ & \\
Unit cell dimensions & $\mathrm{a}=7.3183(11) \AA \quad \quad \quad \alpha=69.766(2)^{\circ}$. \\
& $\mathrm{b}=10.0305(15) \AA$ & $\beta=77.720(2)^{\circ}$. \\
& $\mathrm{c}=10.9470(17) \AA$ & $\gamma=85037(2)^{\circ}$ \\
Volume & $736.66(19) \AA$ & \\
Z, Calculated density & $2,1.138 \mathrm{Mg} / \mathrm{m}^{3}$ & \\
Absorption coefficient & $0.068 \mathrm{~mm}^{-1}$ & \\
\hline
\end{tabular}




\begin{tabular}{ll}
\hline $\mathrm{F}(000)$ & 272 \\
Crystal size & $0.32 \times 0.26 \times 0.22 \mathrm{~mm}$ \\
$\theta$ range for data collection & 2.02 to $25.15^{\circ}$. \\
Limiting indices & $-8<=\mathrm{h}<=8,-11<=\mathrm{k}<=11,-13<=\mathrm{l}<=13$ \\
Reflections collected / unique & $5864 / 2615[\mathrm{R}(\mathrm{int})=0.0319]$ \\
Completeness to $\theta=25.15$ & $99.4 \%$ \\
Max. and min. transmission & 0.9851 and 0.9784 \\
Refinement method & Full-matrix least-squares on $\mathrm{F}^{2}$ \\
Data / restraints / parameters & $2615 / 0 / 176$ \\
Goodness-of-fit on $\mathrm{F}^{2}$ & 1.040 \\
Final R indices [I $>2 \sigma(\mathrm{I})]$ & $\mathrm{R} 1=0.0551, \mathrm{wR} 2=0.1468$ \\
$\mathrm{R}$ indices (all data) & $\mathrm{R} 1=0.0972, \mathrm{wR} 2=0.1770$ \\
Largest diff. peak and hole & 0.165 and $-0.169 \mathrm{e} . \AA^{-3}$ \\
\hline
\end{tabular}

Table 13. Atomic Coordinates $\left(\times 10^{4}\right)$ and Equivalent Isotropic Displacement Parameters $\left(\AA^{2} \times 10^{3}\right)$ for 5ab

\begin{tabular}{|c|c|c|c|c|}
\hline & $\mathrm{x}$ & $\mathrm{y}$ & $\mathrm{z}$ & $\mathrm{U}(\mathrm{eq})^{a}$ \\
\hline$C(1)$ & $6071(3)$ & $3031(2)$ & $5397(2)$ & $70(1)$ \\
\hline$C(2)$ & 4784(3) & 2824(3) & $6559(2)$ & $81(1)$ \\
\hline$C(3)$ & $4349(4)$ & 1494(3) & $7407(3)$ & $87(1)$ \\
\hline$C(4)$ & $5253(5)$ & $363(3)$ & $7107(3)$ & 111(1) \\
\hline$C(5)$ & $6539(4)$ & $568(3)$ & $5941(3)$ & $94(1)$ \\
\hline$C(6)$ & $6974(3)$ & $1915(2)$ & $5040(2)$ & $57(1)$ \\
\hline$C(7)$ & $8314(3)$ & $2155(2)$ & $3764(2)$ & $62(1)$ \\
\hline $\mathrm{C}(8)$ & $8998(4)$ & 1107(3) & $3321(3)$ & $103(1)$ \\
\hline $\mathrm{C}(9)$ & $8872(3)$ & $3658(2)$ & $2943(2)$ & $55(1)$ \\
\hline$C(10)$ & $7953(3)$ & $4445(2)$ & $1902(2)$ & $57(1)$ \\
\hline $\mathrm{C}(11)$ & $8467(3)$ & $5847(2)$ & $1134(2)$ & $56(1)$ \\
\hline$C(12)$ & 9884(3) & $6449(2)$ & $1434(2)$ & $59(1)$ \\
\hline$C(13)$ & $10800(3)$ & $5682(2)$ & $2449(2)$ & $61(1)$ \\
\hline$C(14)$ & $10302(3)$ & $4285(2)$ & $3210(2)$ & $59(1)$ \\
\hline$C(15)$ & $6380(3)$ & $3794(3)$ & $1617(3)$ & $80(1)$ \\
\hline$C(16)$ & $7506(3)$ & $6701(3)$ & $11(2)$ & $77(1)$ \\
\hline $\mathrm{C}(17)$ & $11336(3)$ & $3477(3)$ & 4313(3) & $80(1)$ \\
\hline $\mathrm{C}(18)$ & $11586(4)$ & $8552(3)$ & 1013(3) & 101(1) \\
\hline $\mathrm{O}(1)$ & $10296(2)$ & $7847(2)$ & $666(2)$ & $77(1)$ \\
\hline
\end{tabular}

${ }^{a} \mathrm{U}(\mathrm{eq})$ is defined as one third of the trace of the orthogonalized Uij tensor.

Table 14. Bond Lengths $[\AA]$ and Angles $\left[{ }^{\circ}\right]$ for $\mathbf{5 a b}$

\begin{tabular}{|c|c|}
\hline$C(1)-C(2)$ & $1.375(3)$ \\
\hline$C(1)-C(6)$ & $1.381(3)$ \\
\hline $\mathrm{C}(1)-\mathrm{H}(1)$ & 0.9300 \\
\hline $\mathrm{C}(2)-\mathrm{C}(3)$ & $1.354(3)$ \\
\hline
\end{tabular}




\begin{tabular}{|c|c|}
\hline $\mathrm{C}(2)-\mathrm{H}(2)$ & 0.9300 \\
\hline$C(3)-C(4)$ & $1.367(4)$ \\
\hline $\mathrm{C}(3)-\mathrm{H}(3)$ & 0.9300 \\
\hline$C(4)-C(5)$ & $1.378(4)$ \\
\hline $\mathrm{C}(4)-\mathrm{H}(4)$ & 0.9300 \\
\hline$C(5)-C(6)$ & $1.385(3)$ \\
\hline $\mathrm{C}(5)-\mathrm{H}(5)$ & 0.9300 \\
\hline $\mathrm{C}(6)-\mathrm{C}(7)$ & $1.481(3)$ \\
\hline$C(7)-C(8)$ & $1.323(3)$ \\
\hline$C(7)-C(9)$ & $1.504(3)$ \\
\hline $\mathrm{C}(8)-\mathrm{H}(8 \mathrm{~A})$ & 0.9300 \\
\hline $\mathrm{C}(8)-\mathrm{H}(8 \mathrm{~B})$ & 0.9300 \\
\hline$C(9)-C(14)$ & $1.395(3)$ \\
\hline $\mathrm{C}(9)-\mathrm{C}(10)$ & $1.410(3)$ \\
\hline $\mathrm{C}(10)-\mathrm{C}(11)$ & $1.401(3)$ \\
\hline$C(10)-C(15)$ & $1.508(3)$ \\
\hline $\mathrm{C}(11)-\mathrm{C}(12)$ & $1.392(3)$ \\
\hline $\mathrm{C}(11)-\mathrm{C}(16)$ & $1.508(3)$ \\
\hline$C(12)-C(13)$ & $1.384(3)$ \\
\hline $\mathrm{C}(12)-\mathrm{O}(1)$ & $1.383(2)$ \\
\hline$C(13)-C(14)$ & $1.393(3)$ \\
\hline $\mathrm{C}(13)-\mathrm{H}(13)$ & 0.9300 \\
\hline $\mathrm{C}(14)-\mathrm{C}(17)$ & $1.514(3)$ \\
\hline $\mathrm{C}(15)-\mathrm{H}(15 \mathrm{~A})$ & 0.9600 \\
\hline $\mathrm{C}(15)-\mathrm{H}(15 \mathrm{~B})$ & 0.9600 \\
\hline $\mathrm{C}(15)-\mathrm{H}(15 \mathrm{C})$ & 0.9600 \\
\hline$C(16)-H(16 A)$ & 0.9600 \\
\hline $\mathrm{C}(16)-\mathrm{H}(16 \mathrm{~B})$ & 0.9600 \\
\hline $\mathrm{C}(16)-\mathrm{H}(16 \mathrm{C})$ & 0.9600 \\
\hline $\mathrm{C}(17)-\mathrm{H}(17 \mathrm{~A})$ & 0.9600 \\
\hline $\mathrm{C}(17)-\mathrm{H}(17 \mathrm{~B})$ & 0.9600 \\
\hline $\mathrm{C}(17)-\mathrm{H}(17 \mathrm{C})$ & 0.9600 \\
\hline $\mathrm{C}(18)-\mathrm{O}(1)$ & $1.411(3)$ \\
\hline $\mathrm{C}(18)-\mathrm{H}(18 \mathrm{~A})$ & 0.9600 \\
\hline $\mathrm{C}(18)-\mathrm{H}(18 \mathrm{~B})$ & 0.9600 \\
\hline $\mathrm{C}(18)-\mathrm{H}(18 \mathrm{C})$ & 0.9600 \\
\hline $\mathrm{C}(2)-\mathrm{C}(1)-\mathrm{C}(6)$ & $122.3(2)$ \\
\hline $\mathrm{C}(2)-\mathrm{C}(1)-\mathrm{H}(1)$ & 118.8 \\
\hline $\mathrm{C}(6)-\mathrm{C}(1)-\mathrm{H}(1)$ & 118.8 \\
\hline $\mathrm{C}(3)-\mathrm{C}(2)-\mathrm{C}(1)$ & $120.6(3)$ \\
\hline $\mathrm{C}(3)-\mathrm{C}(2)-\mathrm{H}(2)$ & 119.7 \\
\hline $\mathrm{C}(1)-\mathrm{C}(2)-\mathrm{H}(2)$ & 119.7 \\
\hline $\mathrm{C}(2)-\mathrm{C}(3)-\mathrm{C}(4)$ & $118.8(3)$ \\
\hline
\end{tabular}




\begin{tabular}{|c|c|}
\hline $\mathrm{C}(2)-\mathrm{C}(3)-\mathrm{H}(3)$ & 120.6 \\
\hline $\mathrm{C}(4)-\mathrm{C}(3)-\mathrm{H}(3)$ & 120.6 \\
\hline $\mathrm{C}(3)-\mathrm{C}(4)-\mathrm{C}(5)$ & $120.7(3)$ \\
\hline $\mathrm{C}(3)-\mathrm{C}(4)-\mathrm{H}(4)$ & 119.7 \\
\hline $\mathrm{C}(5)-\mathrm{C}(4)-\mathrm{H}(4)$ & 119.7 \\
\hline$C(4)-C(5)-C(6)$ & $121.7(3)$ \\
\hline $\mathrm{C}(4)-\mathrm{C}(5)-\mathrm{H}(5)$ & 119.2 \\
\hline $\mathrm{C}(6)-\mathrm{C}(5)-\mathrm{H}(5)$ & 119.2 \\
\hline$C(1)-C(6)-C(5)$ & $115.9(2)$ \\
\hline$C(1)-C(6)-C(7)$ & $121.6(2)$ \\
\hline$C(5)-C(6)-C(7)$ & $122.5(2)$ \\
\hline$C(8)-C(7)-C(6)$ & $122.6(2)$ \\
\hline $\mathrm{C}(8)-\mathrm{C}(7)-\mathrm{C}(9)$ & $119.5(2)$ \\
\hline $\mathrm{C}(6)-\mathrm{C}(7)-\mathrm{C}(9)$ & $117.93(18)$ \\
\hline $\mathrm{C}(7)-\mathrm{C}(8)-\mathrm{H}(8 \mathrm{~A})$ & 120.0 \\
\hline $\mathrm{C}(7)-\mathrm{C}(8)-\mathrm{H}(8 \mathrm{~B})$ & 120.0 \\
\hline $\mathrm{H}(8 \mathrm{~A})-\mathrm{C}(8)-\mathrm{H}(8 \mathrm{~B})$ & 120.0 \\
\hline $\mathrm{C}(14)-\mathrm{C}(9)-\mathrm{C}(10)$ & $119.5(2)$ \\
\hline $\mathrm{C}(14)-\mathrm{C}(9)-\mathrm{C}(7)$ & $120.19(19)$ \\
\hline $\mathrm{C}(10)-\mathrm{C}(9)-\mathrm{C}(7)$ & $120.31(19)$ \\
\hline$C(11)-C(10)-C(9)$ & $120.58(19)$ \\
\hline$C(11)-C(10)-C(15)$ & $119.18(19)$ \\
\hline $\mathrm{C}(9)-\mathrm{C}(10)-\mathrm{C}(15)$ & $120.2(2)$ \\
\hline$C(12)-C(11)-C(10)$ & $118.60(19)$ \\
\hline $\mathrm{C}(12)-\mathrm{C}(11)-\mathrm{C}(16)$ & $120.1(2)$ \\
\hline$C(10)-C(11)-C(16)$ & $121.3(2)$ \\
\hline $\mathrm{C}(13)-\mathrm{C}(12)-\mathrm{O}(1)$ & $122.8(2)$ \\
\hline $\mathrm{C}(13)-\mathrm{C}(12)-\mathrm{C}(11)$ & $121.2(2)$ \\
\hline $\mathrm{O}(1)-\mathrm{C}(12)-\mathrm{C}(11)$ & $116.03(19)$ \\
\hline $\mathrm{C}(12)-\mathrm{C}(13)-\mathrm{C}(14)$ & $120.4(2)$ \\
\hline $\mathrm{C}(12)-\mathrm{C}(13)-\mathrm{H}(13)$ & 119.8 \\
\hline $\mathrm{C}(14)-\mathrm{C}(13)-\mathrm{H}(13)$ & 119.8 \\
\hline $\mathrm{C}(13)-\mathrm{C}(14)-\mathrm{C}(9)$ & $119.7(2)$ \\
\hline$C(13)-C(14)-C(17)$ & $119.0(2)$ \\
\hline $\mathrm{C}(9)-\mathrm{C}(14)-\mathrm{C}(17)$ & $121.3(2)$ \\
\hline $\mathrm{C}(10)-\mathrm{C}(15)-\mathrm{H}(15 \mathrm{~A})$ & 109.5 \\
\hline $\mathrm{C}(10)-\mathrm{C}(15)-\mathrm{H}(15 \mathrm{~B})$ & 109.5 \\
\hline $\mathrm{H}(15 \mathrm{~A})-\mathrm{C}(15)-\mathrm{H}(15 \mathrm{~B})$ & 109.5 \\
\hline $\mathrm{C}(10)-\mathrm{C}(15)-\mathrm{H}(15 \mathrm{C})$ & 109.5 \\
\hline $\mathrm{H}(15 \mathrm{~A})-\mathrm{C}(15)-\mathrm{H}(15 \mathrm{C})$ & 109.5 \\
\hline $\mathrm{H}(15 \mathrm{~B})-\mathrm{C}(15)-\mathrm{H}(15 \mathrm{C})$ & 109.5 \\
\hline $\mathrm{C}(11)-\mathrm{C}(16)-\mathrm{H}(16 \mathrm{~A})$ & 109.5 \\
\hline $\mathrm{C}(11)-\mathrm{C}(16)-\mathrm{H}(16 \mathrm{~B})$ & 109.5 \\
\hline $\mathrm{H}(16 \mathrm{~A})-\mathrm{C}(16)-\mathrm{H}(16 \mathrm{~B})$ & 109.5 \\
\hline
\end{tabular}




\begin{tabular}{ll}
\hline $\mathrm{C}(11)-\mathrm{C}(16)-\mathrm{H}(16 \mathrm{C})$ & 109.5 \\
$\mathrm{H}(16 \mathrm{~A})-\mathrm{C}(16)-\mathrm{H}(16 \mathrm{C})$ & 109.5 \\
$\mathrm{H}(16 \mathrm{~B})-\mathrm{C}(16)-\mathrm{H}(16 \mathrm{C})$ & 109.5 \\
$\mathrm{C}(14)-\mathrm{C}(17)-\mathrm{H}(17 \mathrm{~A})$ & 109.5 \\
$\mathrm{C}(14)-\mathrm{C}(17)-\mathrm{H}(17 \mathrm{~B})$ & 109.5 \\
$\mathrm{H}(17 \mathrm{~A})-\mathrm{C}(17)-\mathrm{H}(17 \mathrm{~B})$ & 109.5 \\
$\mathrm{C}(14)-\mathrm{C}(17)-\mathrm{H}(17 \mathrm{C})$ & 109.5 \\
$\mathrm{H}(17 \mathrm{~A})-\mathrm{C}(17)-\mathrm{H}(17 \mathrm{C})$ & 109.5 \\
$\mathrm{H}(17 \mathrm{~B})-\mathrm{C}(17)-\mathrm{H}(17 \mathrm{C})$ & 109.5 \\
$\mathrm{O}(1)-\mathrm{C}(18)-\mathrm{H}(18 \mathrm{~A})$ & 109.5 \\
$\mathrm{O}(1)-\mathrm{C}(18)-\mathrm{H}(18 \mathrm{~B})$ & 109.5 \\
$\mathrm{H}(18 \mathrm{~A})-\mathrm{C}(18)-\mathrm{H}(18 \mathrm{~B})$ & 109.5 \\
$\mathrm{O}(1)-\mathrm{C}(18)-\mathrm{H}(18 \mathrm{C})$ & 109.5 \\
$\mathrm{H}(18 \mathrm{~A})-\mathrm{C}(18)-\mathrm{H}(18 \mathrm{C})$ & 109.5 \\
$\mathrm{H}(18 \mathrm{~B})-\mathrm{C}(18)-\mathrm{H}(18 \mathrm{C})$ & 109.5 \\
$\mathrm{C}(12)-\mathrm{O}(1)-\mathrm{C}(18)$ & $118.27(18)$ \\
\hline
\end{tabular}

Symmetry transformations used to generate equivalent atoms:

Table 15. Anisotropic Displacement Parameters $\left(\mathrm{A}^{2} \times 10^{3}\right)$ for $5 \mathbf{a b}$

\begin{tabular}{lllllll} 
& $\mathrm{U} 11$ & $\mathrm{U} 22$ & $\mathrm{U} 33$ & $\mathrm{U} 23$ & $\mathrm{U} 13$ & $\mathrm{U} 12$ \\
$\mathrm{C}(1)$ & $83(2)$ & $52(1)$ & $65(2)$ & $-12(1)$ & $-7(1)$ & $7(1)$ \\
$\mathrm{C}(2)$ & $87(2)$ & $74(2)$ & $70(2)$ & $-21(1)$ & $0(1)$ & $12(1)$ \\
$\mathrm{C}(3)$ & $87(2)$ & $89(2)$ & $77(2)$ & $-26(2)$ & $6(1)$ & $-24(2)$ \\
$\mathrm{C}(4)$ & $149(3)$ & $65(2)$ & $97(2)$ & $-16(2)$ & $17(2)$ & $-37(2)$ \\
$\mathrm{C}(5)$ & $117(2)$ & $53(2)$ & $100(2)$ & $-26(2)$ & $7(2)$ & $-13(2)$ \\
$\mathrm{C}(6)$ & $61(1)$ & $47(1)$ & $64(1)$ & $-17(1)$ & $-16(1)$ & $-3(1)$ \\
$\mathrm{C}(7)$ & $68(1)$ & $49(1)$ & $69(2)$ & $-22(1)$ & $-10(1)$ & $5(1)$ \\
$\mathrm{C}(8)$ & $128(2)$ & $62(2)$ & $104(2)$ & $-33(2)$ & $18(2)$ & $-3(2)$ \\
$\mathrm{C}(9)$ & $60(1)$ & $48(1)$ & $56(1)$ & $-20(1)$ & $-7(1)$ & $1(1)$ \\
$\mathrm{C}(10)$ & $57(1)$ & $62(1)$ & $57(1)$ & $-28(1)$ & $-6(1)$ & $-1(1)$ \\
$\mathrm{C}(11)$ & $58(1)$ & $57(1)$ & $51(1)$ & $-20(1)$ & $-7(1)$ & $3(1)$ \\
$\mathrm{C}(12)$ & $63(1)$ & $51(1)$ & $56(1)$ & $-16(1)$ & $-4(1)$ & $-1(1)$ \\
$\mathrm{C}(13)$ & $60(1)$ & $60(1)$ & $65(1)$ & $-24(1)$ & $-12(1)$ & $-6(1)$ \\
$\mathrm{C}(14)$ & $62(1)$ & $57(1)$ & $57(1)$ & $-20(1)$ & $-10(1)$ & $4(1)$ \\
$\mathrm{C}(15)$ & $82(2)$ & $81(2)$ & $87(2)$ & $-35(2)$ & $-22(1)$ & $-12(1)$ \\
$\mathrm{C}(16)$ & $85(2)$ & $80(2)$ & $64(2)$ & $-21(1)$ & $-22(1)$ & $7(1)$ \\
$\mathrm{C}(17)$ & $82(2)$ & $75(2)$ & $85(2)$ & $-20(1)$ & $-34(1)$ & $8(1)$ \\
$\mathrm{C}(18)$ & $104(2)$ & $66(2)$ & $125(2)$ & $-13(2)$ & $-26(2)$ & $-27(2)$ \\
$\mathrm{O}(1)$ & $86(1)$ & $58(1)$ & $79(1)$ & $-10(1)$ & $-16(1)$ & $-11(1)$ \\
\hline
\end{tabular}

The anisotropic displacement factor exponent takes the form: $-2 \mathrm{pi}^{\wedge} 2\left[\mathrm{~h}^{\wedge} 2 \mathrm{a}^{* \wedge} 2 \mathrm{U} 11+\ldots+2 \mathrm{~h} \mathrm{k} \mathrm{a}^{*} \mathrm{~b}^{*}\right.$ U12 ]

Table 16. Hydrogen Coordinates $\left(\times 10^{4}\right)$ and Isotropic Displacement Parameters $\left(\AA^{2} \times 10^{3}\right)$ for $\mathbf{5 a b}$ 


\begin{tabular}{rrrrr} 
& $x$ & $y$ & U (eq) \\
H(1) & 6343 & 3955 & 4831 & 84 \\
$\mathrm{H}(2)$ & 4208 & 3603 & 6765 & 97 \\
$\mathrm{H}(3)$ & 3450 & 1352 & 8180 & 104 \\
$\mathrm{H}(4)$ & 4997 & -554 & 7695 & 133 \\
$\mathrm{H}(5)$ & 7131 & -218 & 5755 & 112 \\
$\mathrm{H}(8 \mathrm{~A})$ & 8641 & 177 & 3815 & 123 \\
$\mathrm{H}(8 \mathrm{~B})$ & 9837 & 1301 & 2515 & 123 \\
$\mathrm{H}(13)$ & 11755 & 6101 & 2625 & 73 \\
$\mathrm{H}(15 \mathrm{~A})$ & 6085 & 2886 & 2290 & 121 \\
$\mathrm{H}(15 \mathrm{~B})$ & 6756 & 3675 & 765 & 121 \\
$\mathrm{H}(15 \mathrm{C})$ & 5298 & 4408 & 1614 & 121 \\
$\mathrm{H}(16 \mathrm{~A})$ & 8085 & 7612 & -428 & 115 \\
$\mathrm{H}(16 \mathrm{~B})$ & 6209 & 6831 & 115 \\
$\mathrm{H}(16 \mathrm{C})$ & 7612 & 6205 & 358 & 115 \\
$\mathrm{H}(17 \mathrm{~A})$ & 12271 & 2868 & -610 & 121 \\
$\mathrm{H}(17 \mathrm{~B})$ & 10467 & 2912 & 4011 & 121 \\
$\mathrm{H}(17 \mathrm{C})$ & 11924 & 4137 & 5068 & 121 \\
$\mathrm{H}(18 \mathrm{~A})$ & 11169 & 8516 & 152 \\
$\mathrm{H}(18 \mathrm{~B})$ & 11678 & 9525 & 438 & 152 \\
$\mathrm{H}(18 \mathrm{C})$ & 12790 & 8094 & 921 & 152 \\
\hline & & & \\
\hline
\end{tabular}

Table 17. Torsion Angles $\left[{ }^{\circ}\right]$ for $\mathbf{5 a b}$

\begin{tabular}{rr}
\hline $\mathrm{C}(6)-\mathrm{C}(1)-\mathrm{C}(2)-\mathrm{C}(3)$ & $-0.1(4)$ \\
$\mathrm{C}(1)-\mathrm{C}(2)-\mathrm{C}(3)-\mathrm{C}(4)$ & $2.0(4)$ \\
$\mathrm{C}(2)-\mathrm{C}(3)-\mathrm{C}(4)-\mathrm{C}(5)$ & $-2.2(5)$ \\
$\mathrm{C}(3)-\mathrm{C}(4)-\mathrm{C}(5)-\mathrm{C}(6)$ & $0.4(5)$ \\
$\mathrm{C}(2)-\mathrm{C}(1)-\mathrm{C}(6)-\mathrm{C}(5)$ & $-1.5(3)$ \\
$\mathrm{C}(2)-\mathrm{C}(1)-\mathrm{C}(6)-\mathrm{C}(7)$ & $178.1(2)$ \\
$\mathrm{C}(4)-\mathrm{C}(5)-\mathrm{C}(6)-\mathrm{C}(1)$ & $1.4(4)$ \\
$\mathrm{C}(4)-\mathrm{C}(5)-\mathrm{C}(6)-\mathrm{C}(7)$ & $-178.3(2)$ \\
$\mathrm{C}(1)-\mathrm{C}(6)-\mathrm{C}(7)-\mathrm{C}(8)$ & $-170.6(2)$ \\
$\mathrm{C}(5)-\mathrm{C}(6)-\mathrm{C}(7)-\mathrm{C}(8)$ & $9.0(4)$ \\
$\mathrm{C}(1)-\mathrm{C}(6)-\mathrm{C}(7)-\mathrm{C}(9)$ & $8.2(3)$ \\
$\mathrm{C}(5)-\mathrm{C}(6)-\mathrm{C}(7)-\mathrm{C}(9)$ & $-172.2(2)$ \\
$\mathrm{C}(8)-\mathrm{C}(7)-\mathrm{C}(9)-\mathrm{C}(14)$ & $-97.4(3)$ \\
$\mathrm{C}(6)-\mathrm{C}(7)-\mathrm{C}(9)-\mathrm{C}(14)$ & $83.8(2)$ \\
$\mathrm{C}(8)-\mathrm{C}(7)-\mathrm{C}(9)-\mathrm{C}(10)$ & $82.8(3)$ \\
$\mathrm{C}(6)-\mathrm{C}(7)-\mathrm{C}(9)-\mathrm{C}(10)$ & $-96.0(2)$ \\
$\mathrm{C}(14)-\mathrm{C}(9)-\mathrm{C}(10)-\mathrm{C}(11)$ & $0.2(3)$ \\
$\mathrm{C}(7)-\mathrm{C}(9)-\mathrm{C}(10)-\mathrm{C}(11)$ & $179.95(18)$ \\
$\mathrm{C}(14)-\mathrm{C}(9)-\mathrm{C}(10)-\mathrm{C}(15)$ & $-178.82(19)$ \\
$\mathrm{C}(7)-\mathrm{C}(9)-\mathrm{C}(10)-\mathrm{C}(15)$ & $1.0(3)$ \\
\hline
\end{tabular}




\begin{tabular}{rr}
\hline $\mathrm{C}(9)-\mathrm{C}(10)-\mathrm{C}(11)-\mathrm{C}(12)$ & $-0.9(3)$ \\
$\mathrm{C}(15)-\mathrm{C}(10)-\mathrm{C}(11)-\mathrm{C}(12)$ & $178.13(19)$ \\
$\mathrm{C}(9)-\mathrm{C}(10)-\mathrm{C}(11)-\mathrm{C}(16)$ & $179.62(18)$ \\
$\mathrm{C}(15)-\mathrm{C}(10)-\mathrm{C}(11)-\mathrm{C}(16)$ & $-1.4(3)$ \\
$\mathrm{C}(10)-\mathrm{C}(11)-\mathrm{C}(12)-\mathrm{C}(13)$ & $1.2(3)$ \\
$\mathrm{C}(16)-\mathrm{C}(11)-\mathrm{C}(12)-\mathrm{C}(13)$ & $-179.27(19)$ \\
$\mathrm{C}(10)-\mathrm{C}(11)-\mathrm{C}(12)-\mathrm{O}(1)$ & $-178.31(17)$ \\
$\mathrm{C}(16)-\mathrm{C}(11)-\mathrm{C}(12)-\mathrm{O}(1)$ & $1.2(3)$ \\
$\mathrm{O}(1)-\mathrm{C}(12)-\mathrm{C}(13)-\mathrm{C}(14)$ & $178.64(18)$ \\
$\mathrm{C}(11)-\mathrm{C}(12)-\mathrm{C}(13)-\mathrm{C}(14)$ & $-0.8(3)$ \\
$\mathrm{C}(12)-\mathrm{C}(13)-\mathrm{C}(14)-\mathrm{C}(9)$ & $0.1(3)$ \\
$\mathrm{C}(12)-\mathrm{C}(13)-\mathrm{C}(14)-\mathrm{C}(17)$ & $179.97(19)$ \\
$\mathrm{C}(10)-\mathrm{C}(9)-\mathrm{C}(14)-\mathrm{C}(13)$ & $0.2(3)$ \\
$\mathrm{C}(7)-\mathrm{C}(9)-\mathrm{C}(14)-\mathrm{C}(13)$ & $-179.57(19)$ \\
$\mathrm{C}(10)-\mathrm{C}(9)-\mathrm{C}(14)-\mathrm{C}(17)$ & $-179.63(19)$ \\
$\mathrm{C}(7)-\mathrm{C}(9)-\mathrm{C}(14)-\mathrm{C}(17)$ & $0.6(3)$ \\
$\mathrm{C}(13)-\mathrm{C}(12)-\mathrm{O}(1)-\mathrm{C}(18)$ & $-5.8(3)$ \\
$\mathrm{C}(11)-\mathrm{C}(12)-\mathrm{O}(1)-\mathrm{C}(18)$ & $173.7(2)$ \\
\hline
\end{tabular}

Symmetry transformations used to generate equivalent atoms:

Table 18. Hydrogen Bonds for 5ab $\left[\AA\right.$ and $\left.^{\circ}\right]$

\begin{tabular}{lllll}
\hline $\mathrm{D}-\mathrm{H} . . . \mathrm{A}$ & $\mathrm{d}(\mathrm{D}-\mathrm{H})$ & $\mathrm{d}(\mathrm{H} \ldots \mathrm{A})$ & $\mathrm{d}(\mathrm{D} \ldots \mathrm{A})$ & $<(\mathrm{DHA})$ \\
\hline
\end{tabular}

X-ray structure of compound $\mathbf{5 a b}$
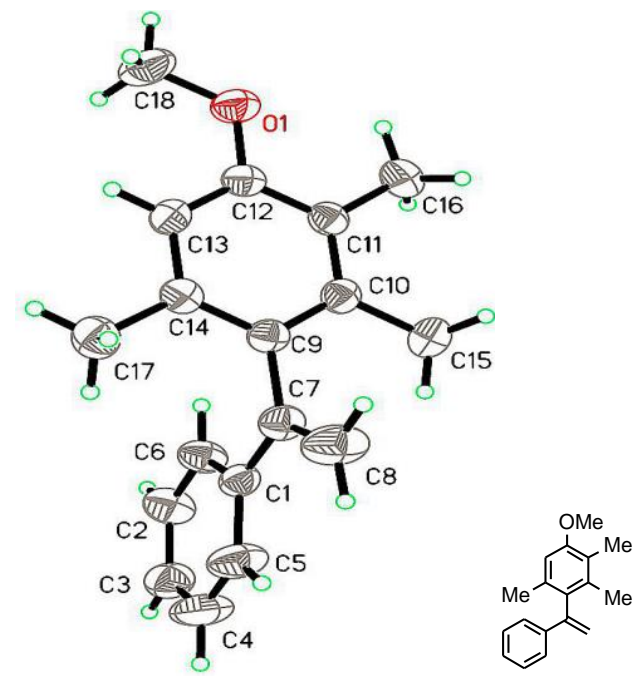

\section{References}

(1) Reich, H. J.; Renga, J. N.; Reich, I. L. J. Am. Chem. Soc. 1975, 97, 5434.

(2) Zhao, L.; Li, Z.; Wirth, T. Eur. J. Org. Chem. 2011, 76, 7080.

(3) Hu, Y. C.; Wang, K. Z.; MacMillan, J. B. Org. Lett. 2013, 15, 390.

(4) Okamoto, K.; Nishibayashi, Y.; Uemura S.; Toshimitsu, A. Angew. Chem., Int. Ed. 2005, 44, 3588.

(5) Wirth, T. Organoselenium Chemistry: Synthesis and Reactions; Wiley-VCH: Weinheim, 2012. 
(6) Tang, E.; Wang, W. L.; Zhao, Y. J.; Zhang, M.; Dai, X. Org. Lett. 2016, 18, 176.

(7) Zhang, S. H.; Qian, P. C.; Zhang, M. L.; Hu, M. L.; Cheng, J. J. Org. Chem. 2010, 75, 6732.

\section{Copies of NMR Spectra for compounds}

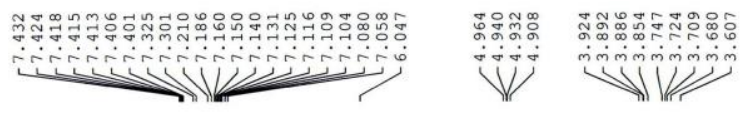

${ }^{1} \mathrm{H}$ NMR $\left(300 \mathrm{MHz}, \mathrm{CDCl}_{3}\right)$
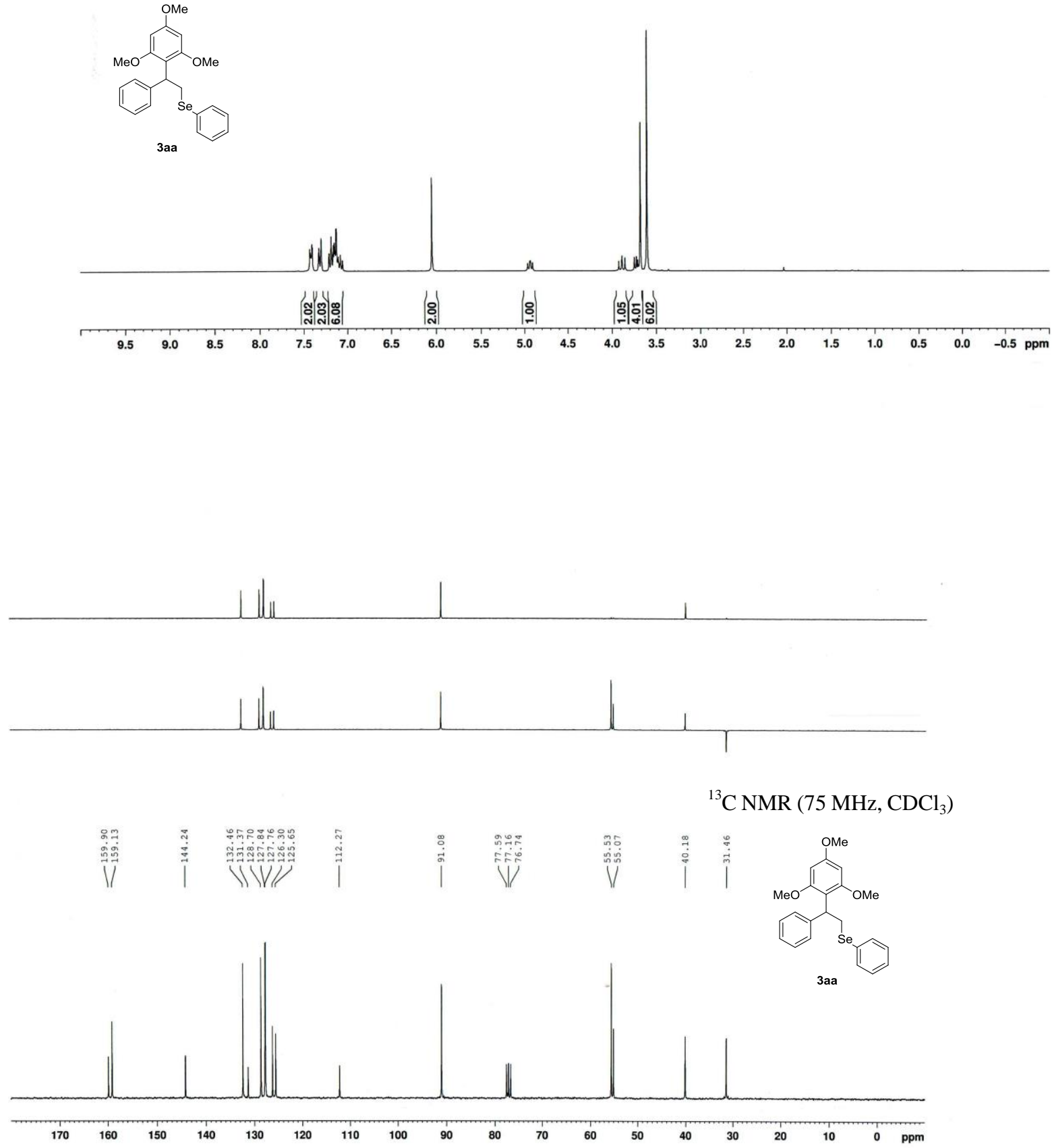

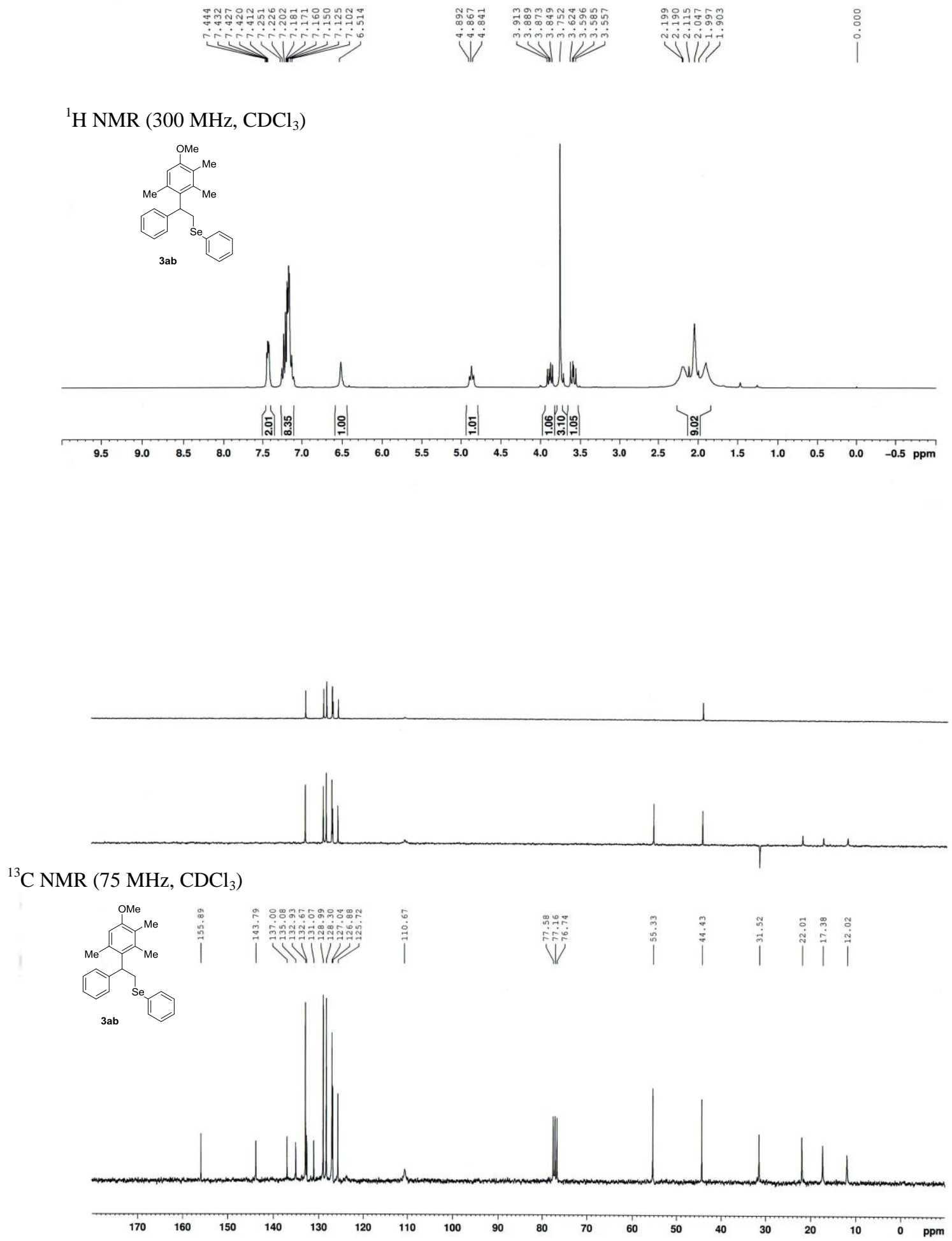


\section{تصن}

${ }^{1} \mathrm{H}$ NMR $\left(300 \mathrm{MHz}, \mathrm{CDCl}_{3}\right)$
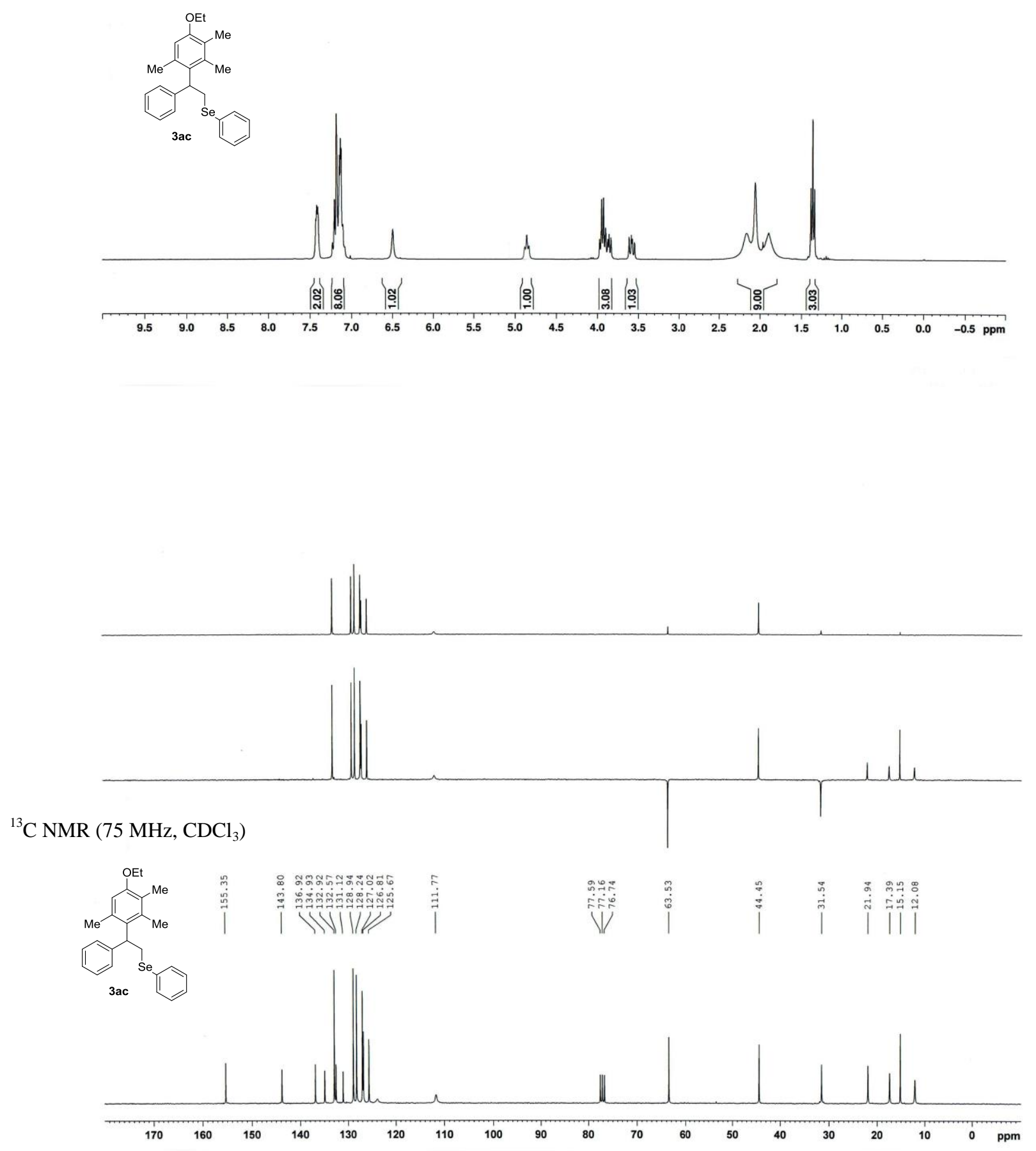


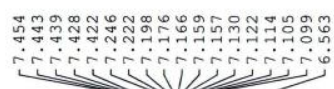

${ }^{1} \mathrm{H}$ NMR $\left(300 \mathrm{MHz}, \mathrm{CDCl}_{3}\right)$
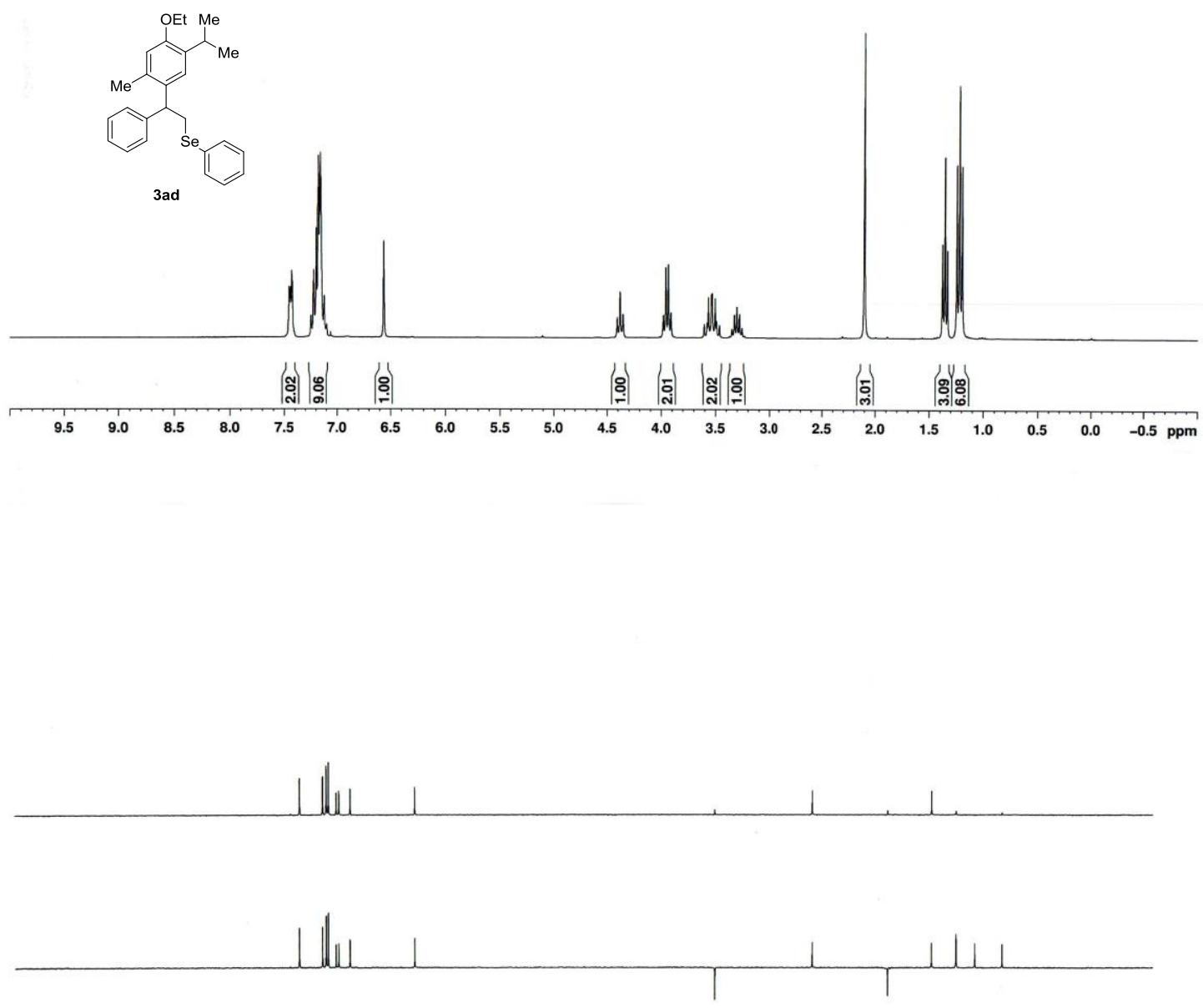

${ }^{13} \mathrm{C}$ NMR $\left(75 \mathrm{MHz}, \mathrm{CDCl}_{3}\right)$

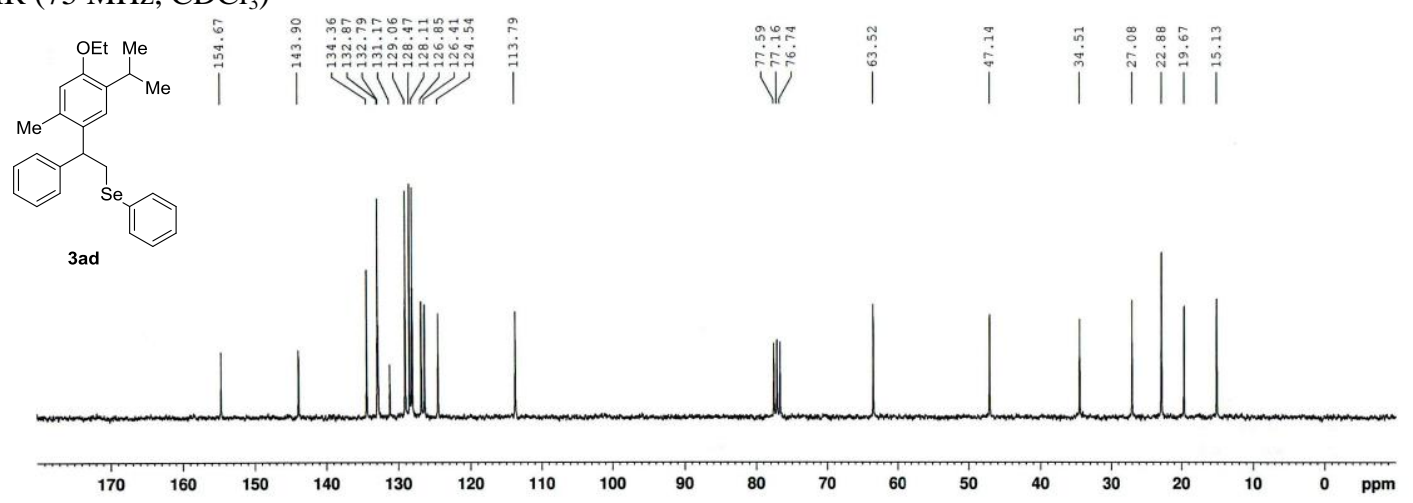



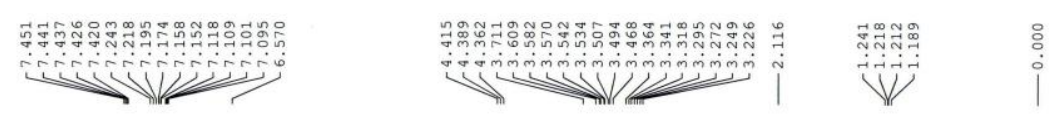

${ }^{1} \mathrm{H}$ NMR $\left(300 \mathrm{MHz}, \mathrm{CDCl}_{3}\right)$

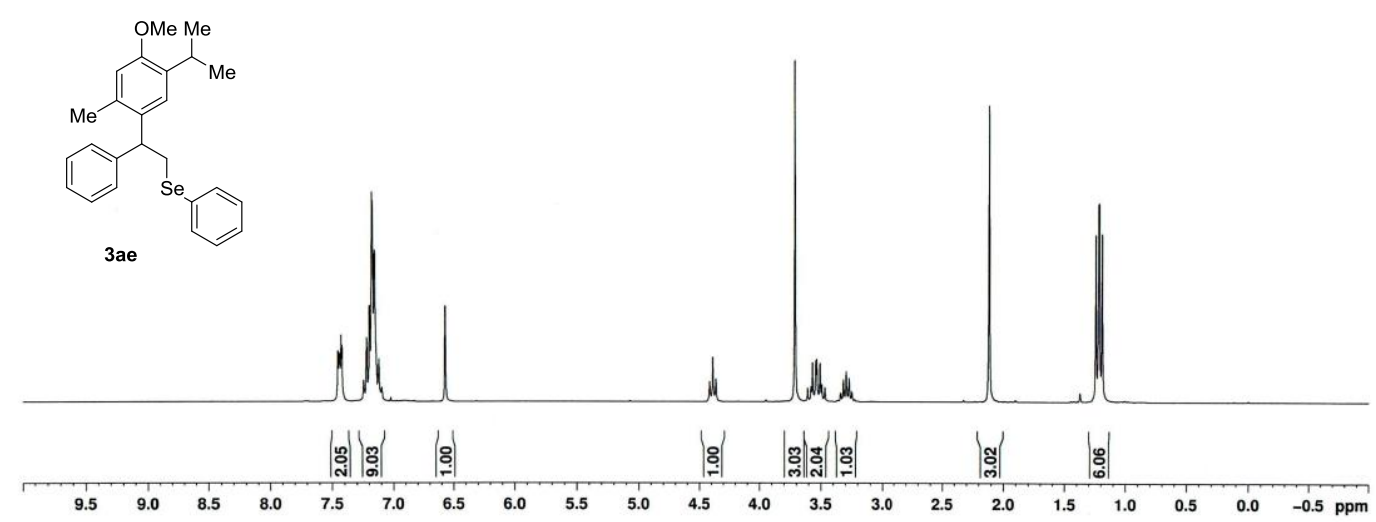

Lلlu

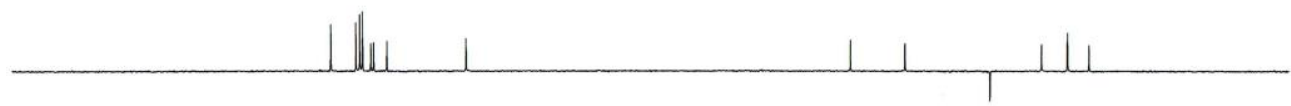

${ }^{13} \mathrm{C}$ NMR $\left(75 \mathrm{MHz}, \mathrm{CDCl}_{3}\right.$ )

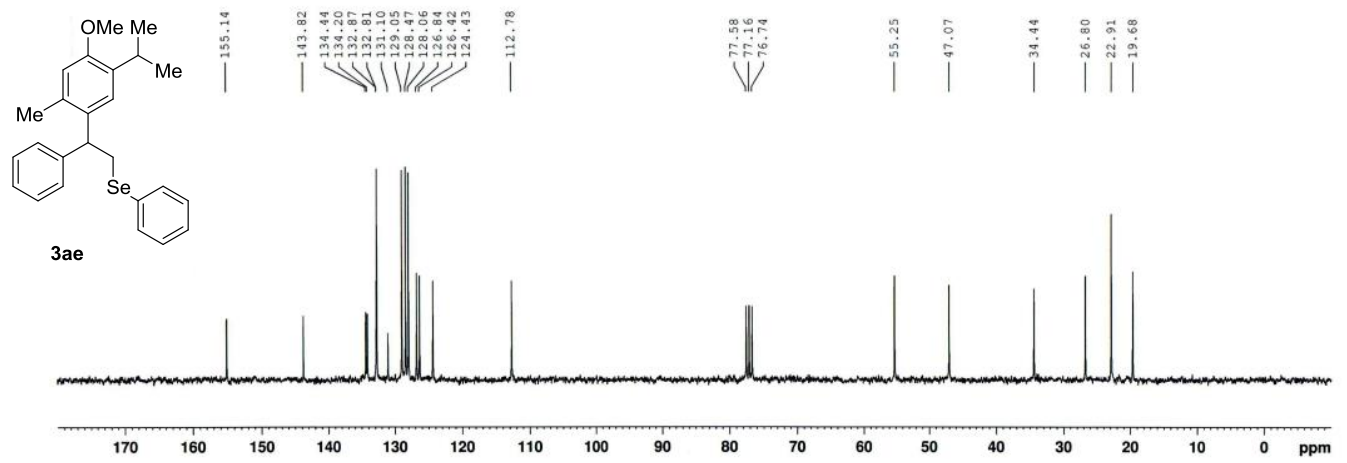


${ }^{1} \mathrm{H}$ NMR $\left(300 \mathrm{MHz}, \mathrm{CDCl}_{3}\right)$
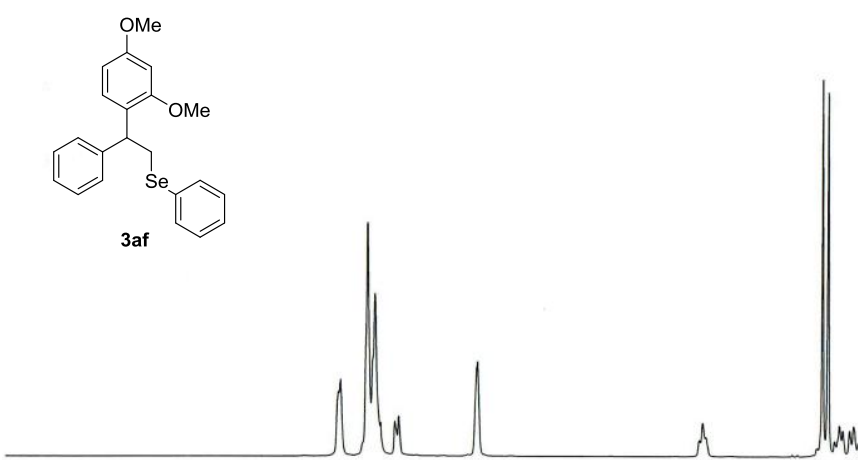

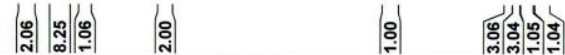

$\begin{array}{lllllllllllllllllllllllllllll}9.5 & 9.0 & 8.5 & 8.0 & 7.5 & 7.0 & 6.5 & 6.0 & 5.5 & 5.0 & 4.5 & 4.0 & 3.5 & 3.0 & 2.5 & 2.0 & 1.5 & 1.0 & 0.5 & 0.0 & -0.5 & \mathrm{ppm}\end{array}$
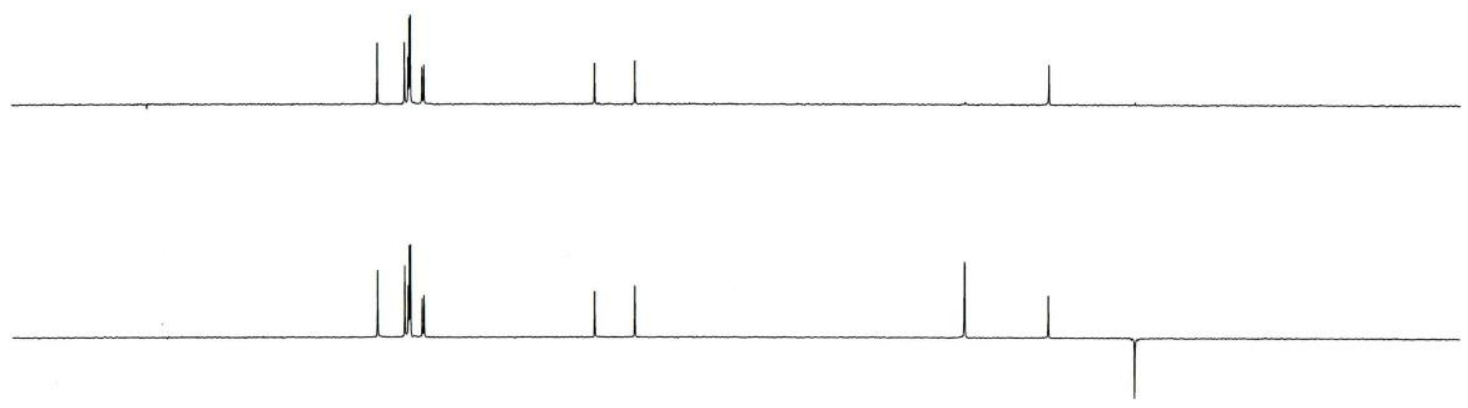

${ }^{13} \mathrm{C}$ NMR $\left(75 \mathrm{MHz}, \mathrm{CDCl}_{3}\right)$

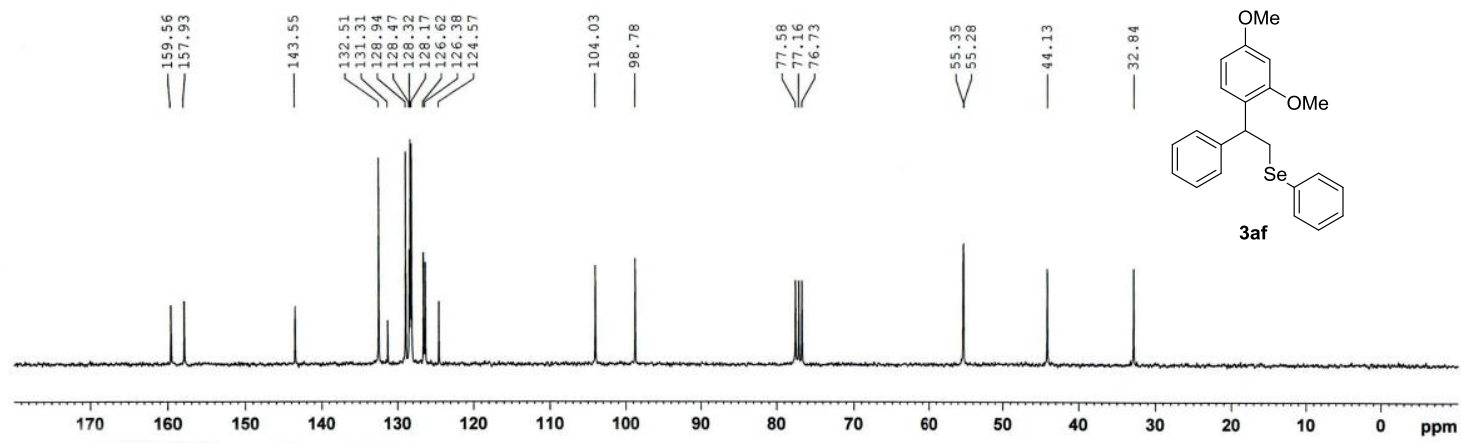




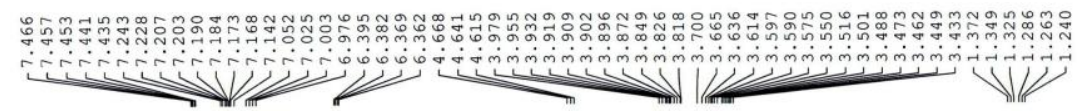

${ }^{1} \mathrm{H}$ NMR (300 MHz, $\mathrm{CDCl}_{3}$ )
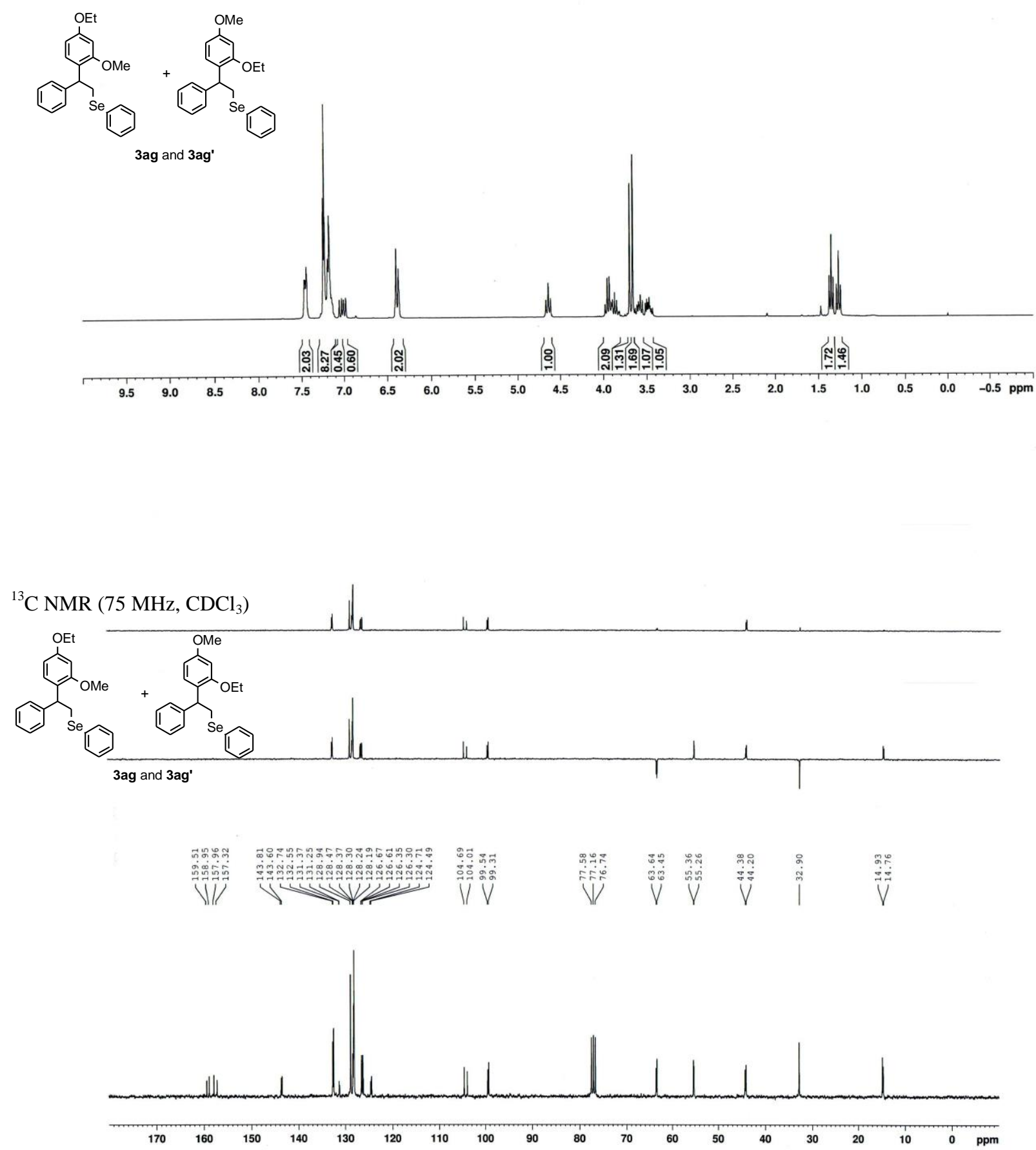


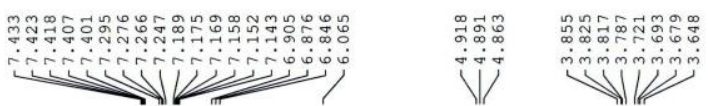

${ }^{1} \mathrm{H}$ NMR $\left(300 \mathrm{MHz}, \mathrm{CDCl}_{3}\right)$

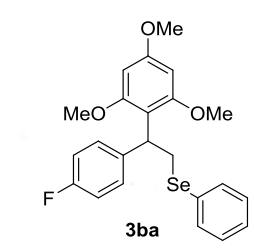

trat

促

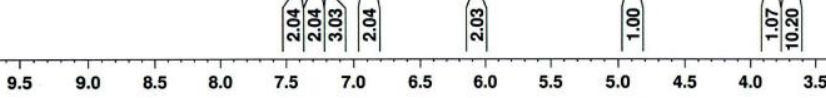

5:

ل ا

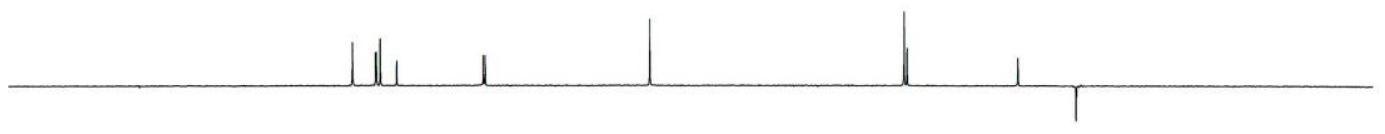

${ }^{13} \mathrm{C}$ NMR $\left(75 \mathrm{MHz}, \mathrm{CDCl}_{3}\right)$

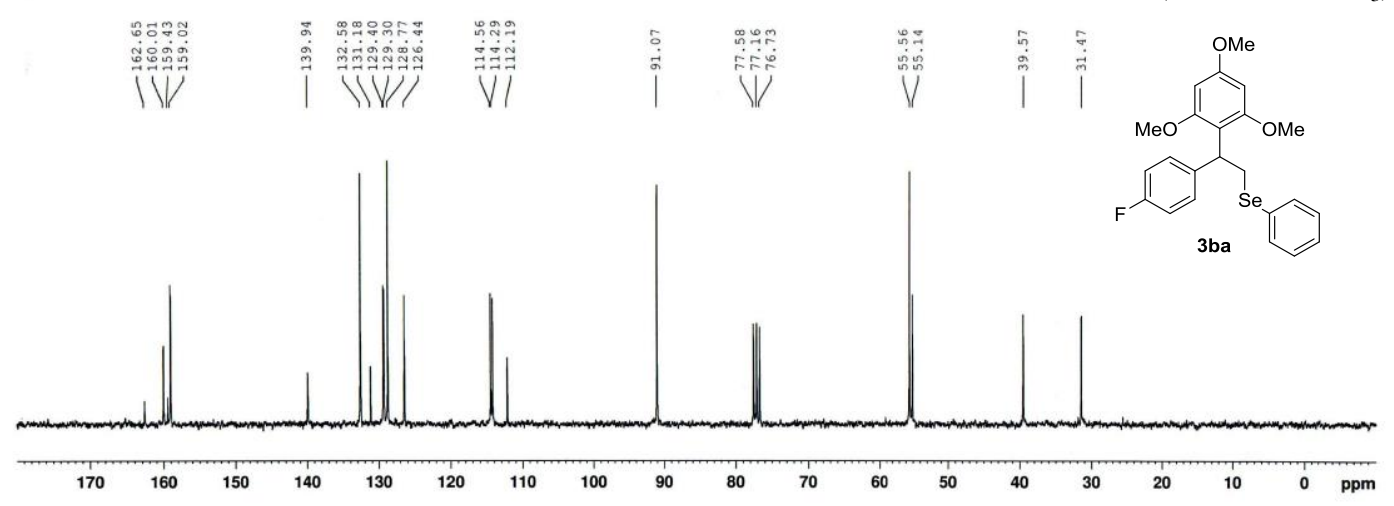




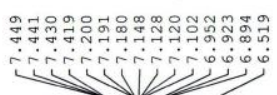

${ }^{1} \mathrm{H}$ NMR $\left(300 \mathrm{MHz}, \mathrm{CDCl}_{3}\right)$
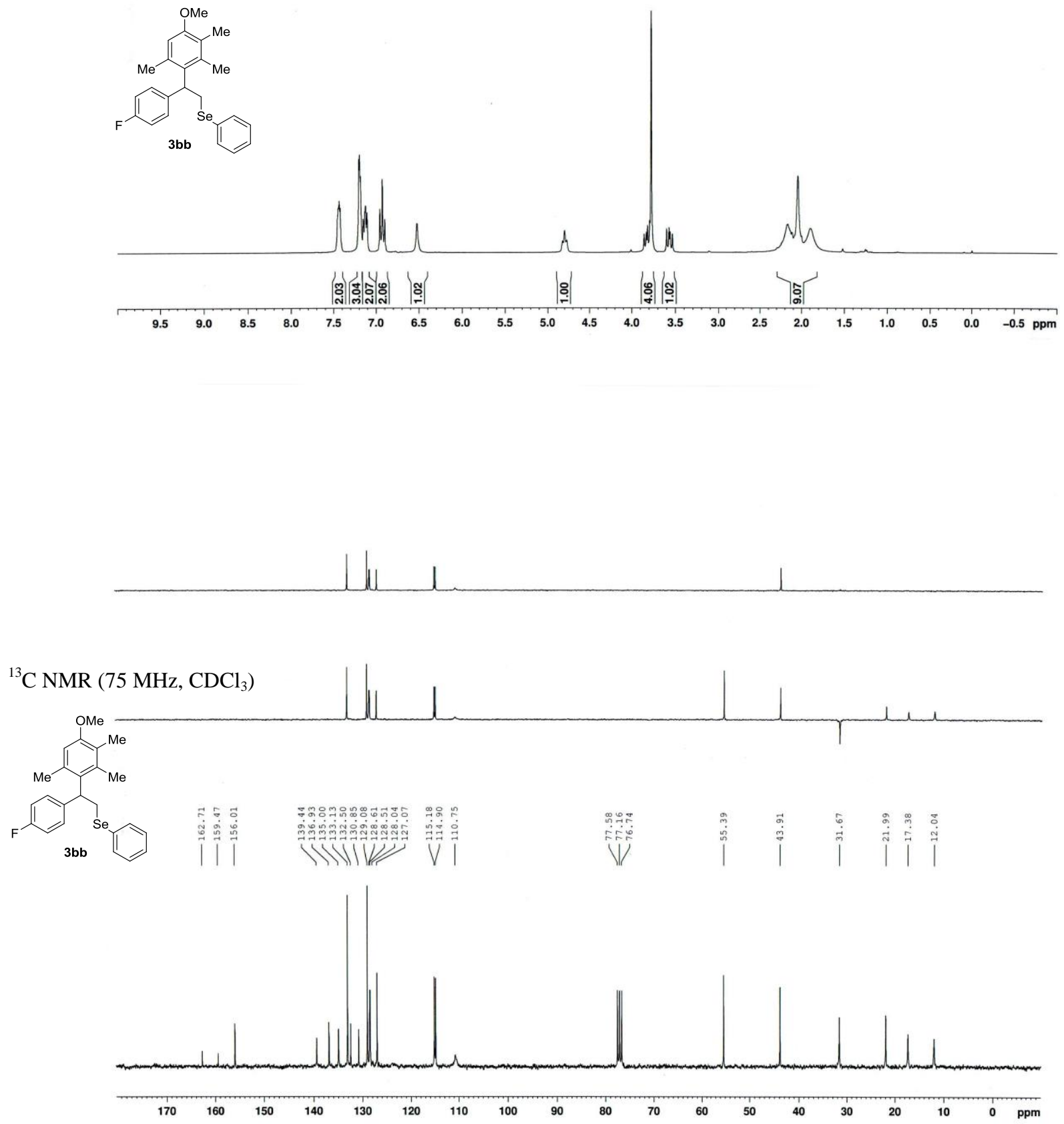

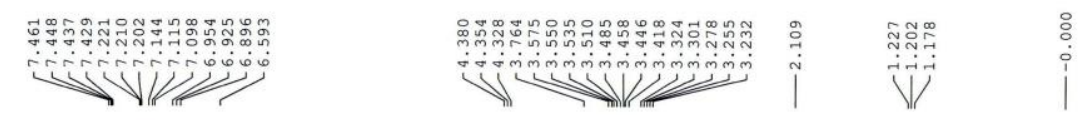

${ }^{1} \mathrm{H} \mathrm{NMR}\left(300 \mathrm{MHz}, \mathrm{CDCl}_{3}\right.$ )
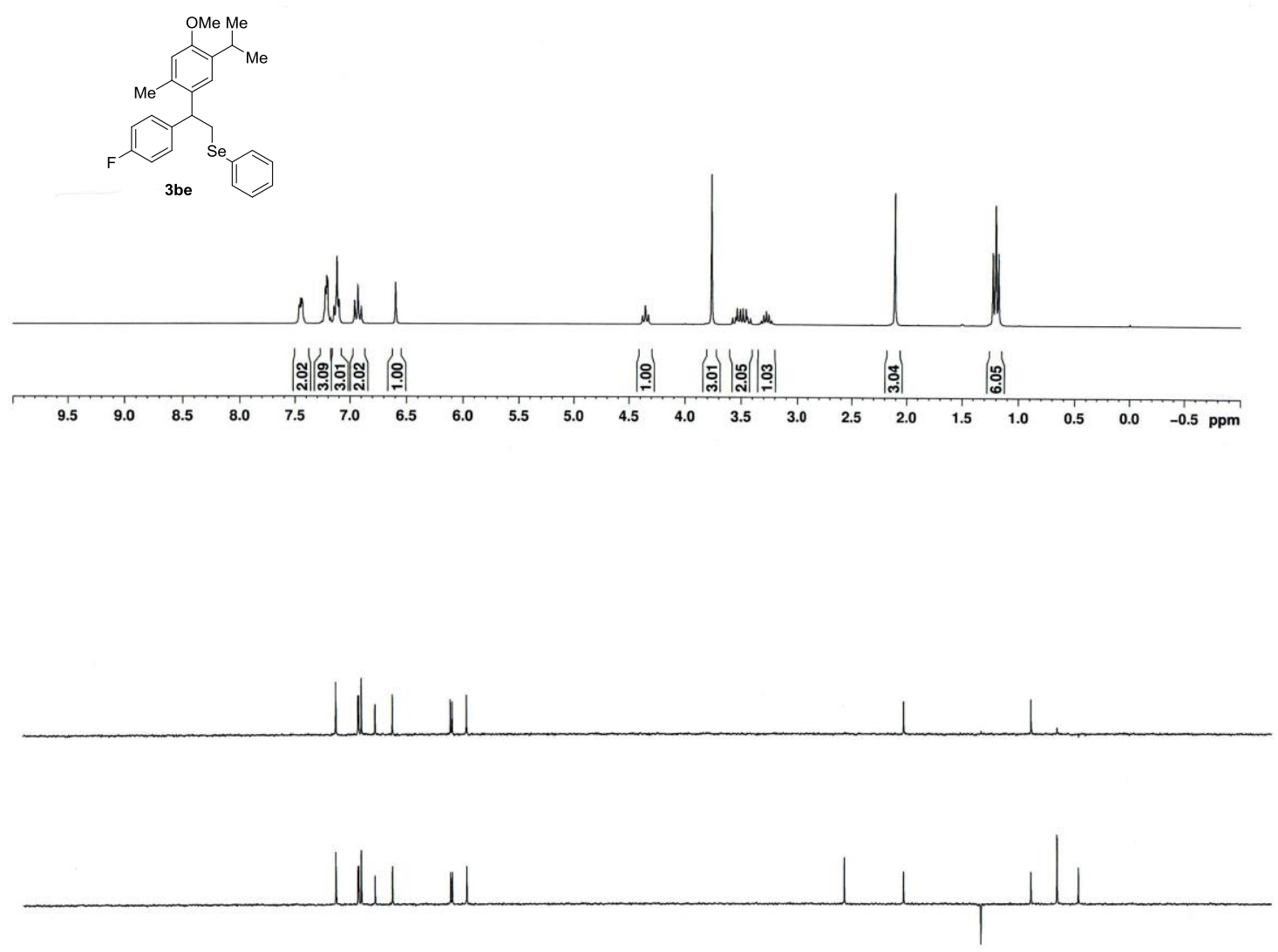

${ }^{13} \mathrm{C}$ NMR (75 MHz, $\mathrm{CDCl}_{3}$ )

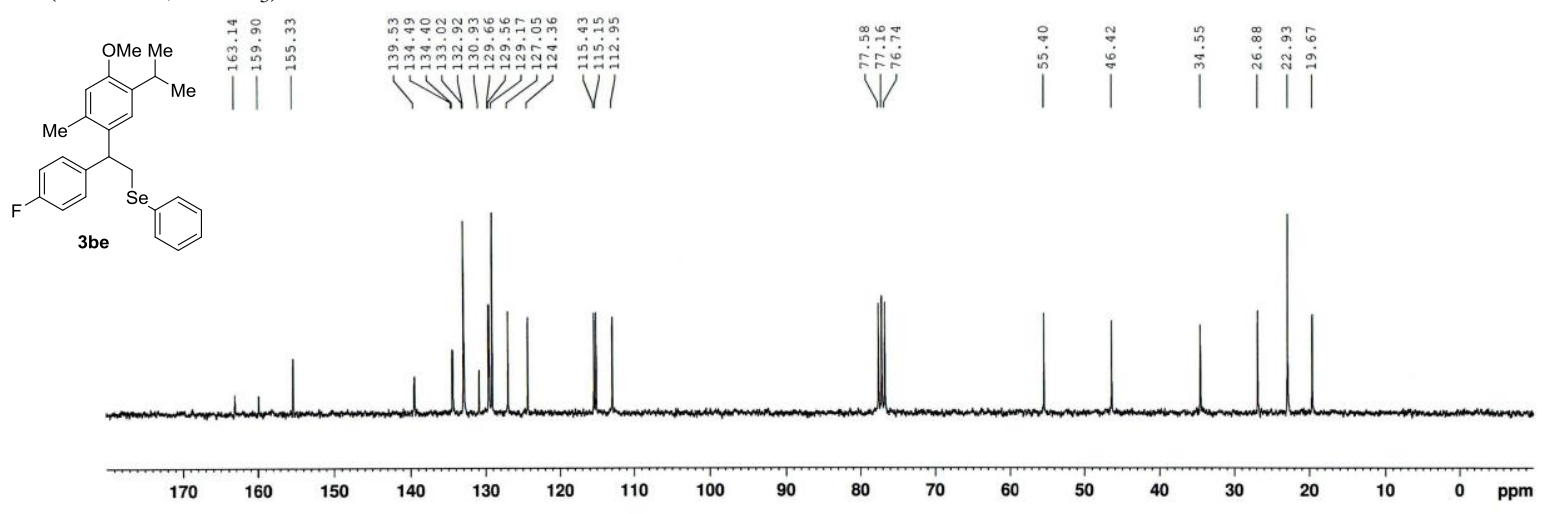


${ }^{1} \mathrm{H}$ NMR (300 MHz, $\mathrm{CDCl}_{3}$ )

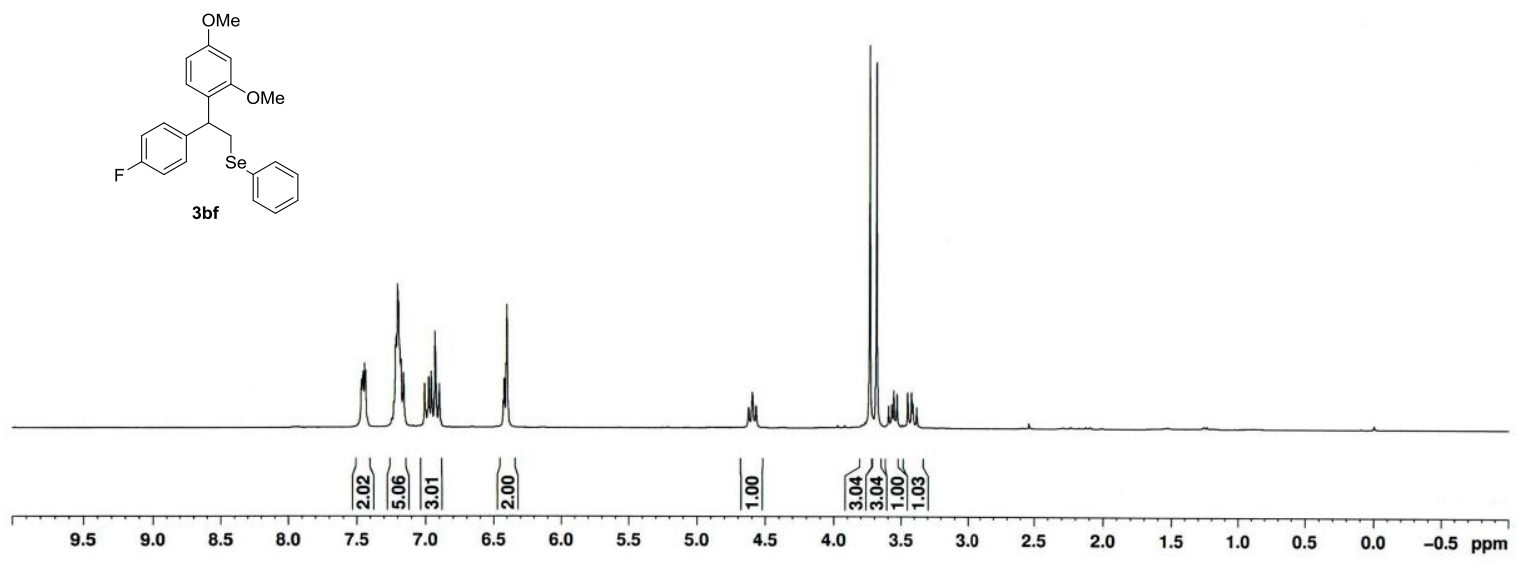

${ }^{13} \mathrm{C}$ NMR $\left(75 \mathrm{MHz}, \mathrm{CDCl}_{3}\right)$
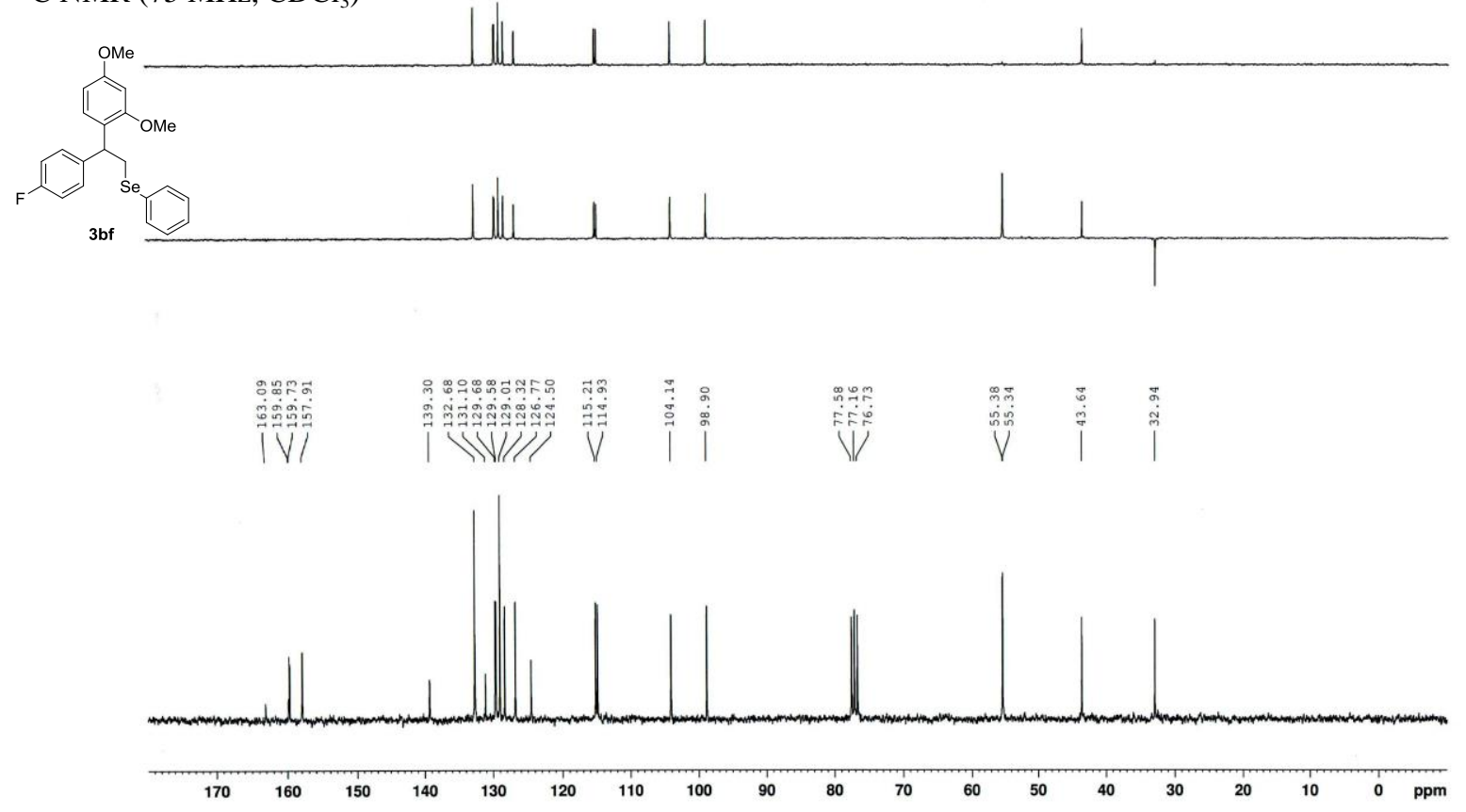


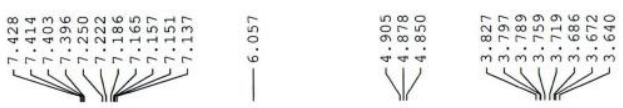

${ }^{1} \mathrm{H}$ NMR $\left(300 \mathrm{MHz}, \mathrm{CDCl}_{3}\right)$
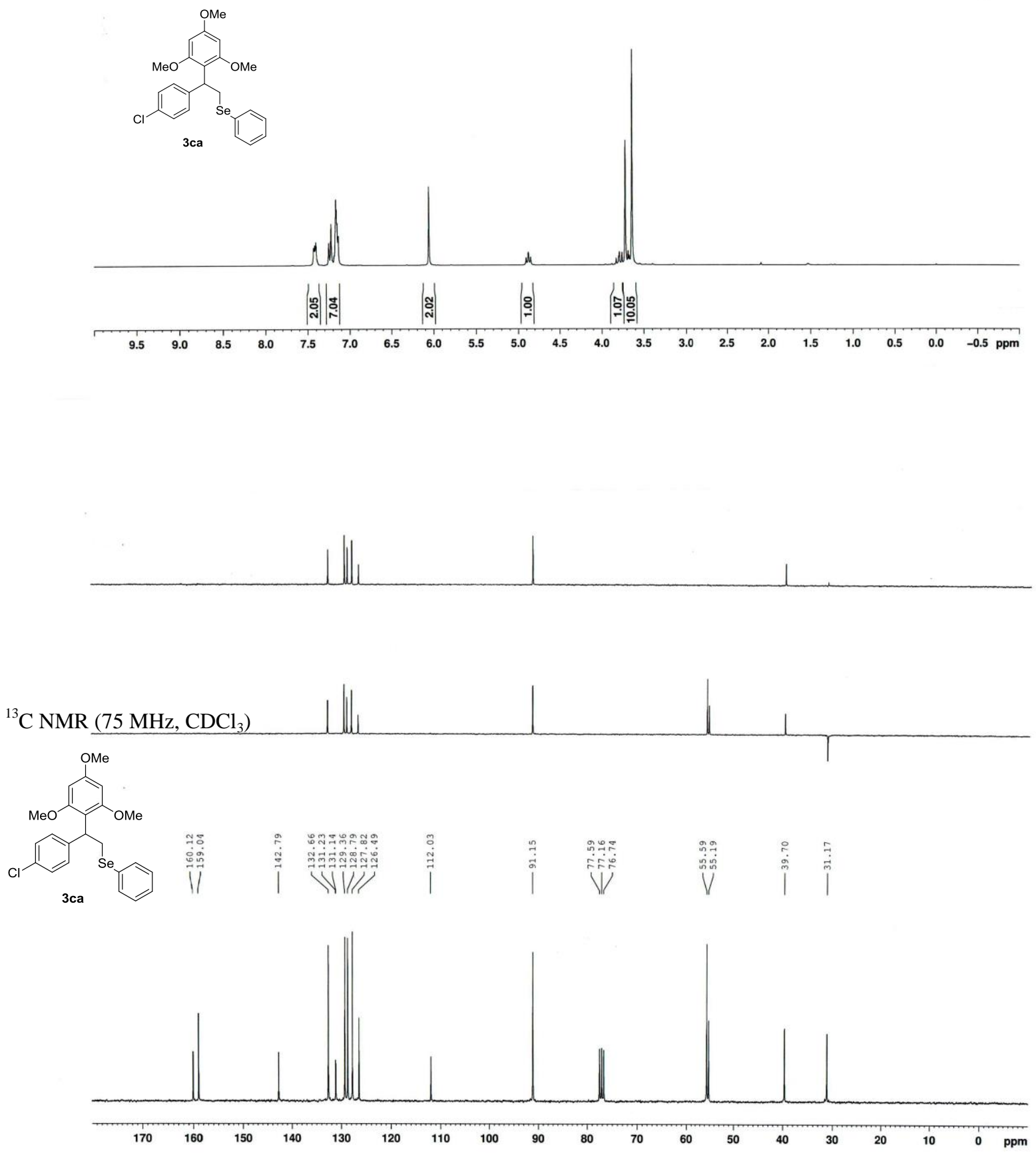
${ }^{1} \mathrm{H}$ NMR (300 MHz, $\mathrm{CDCl}_{3}$ )

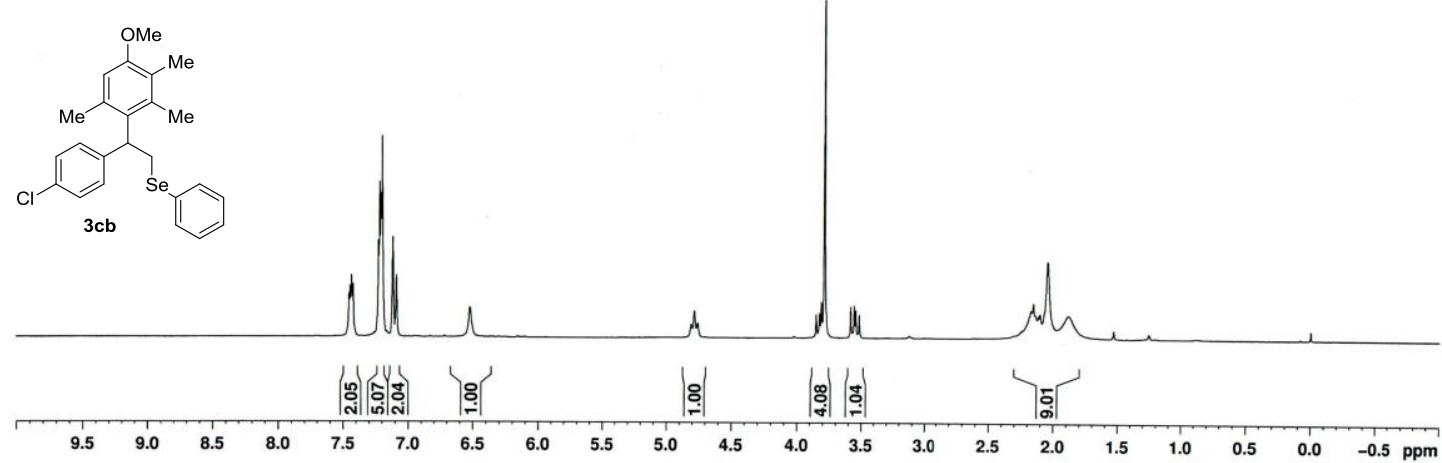

${ }^{13} \mathrm{C}$ NMR (75 MHz, $\mathrm{CDCl}_{3}$ )
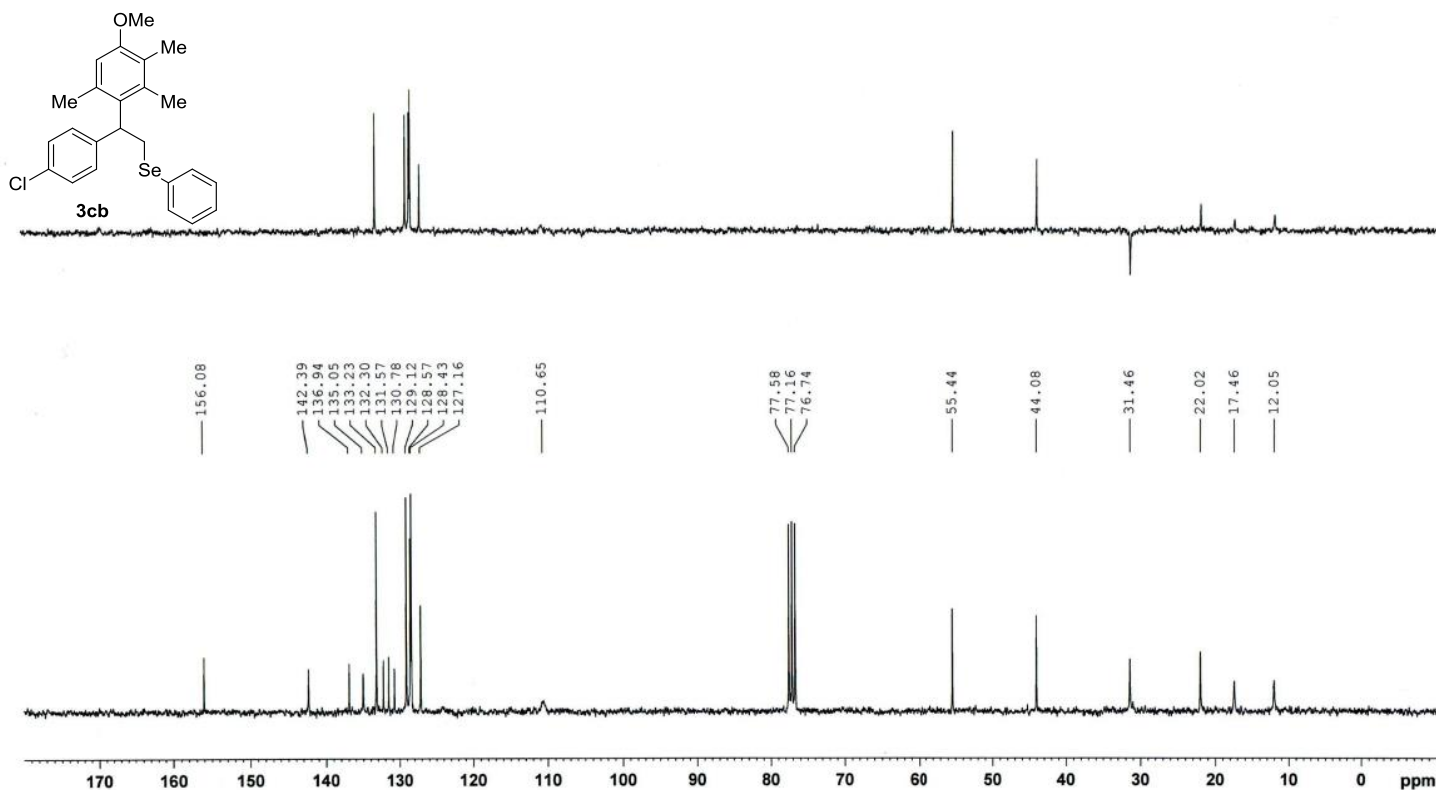
${ }^{1} \mathrm{H}$ NMR (300 MHz, $\mathrm{CDCl}_{3}$ )

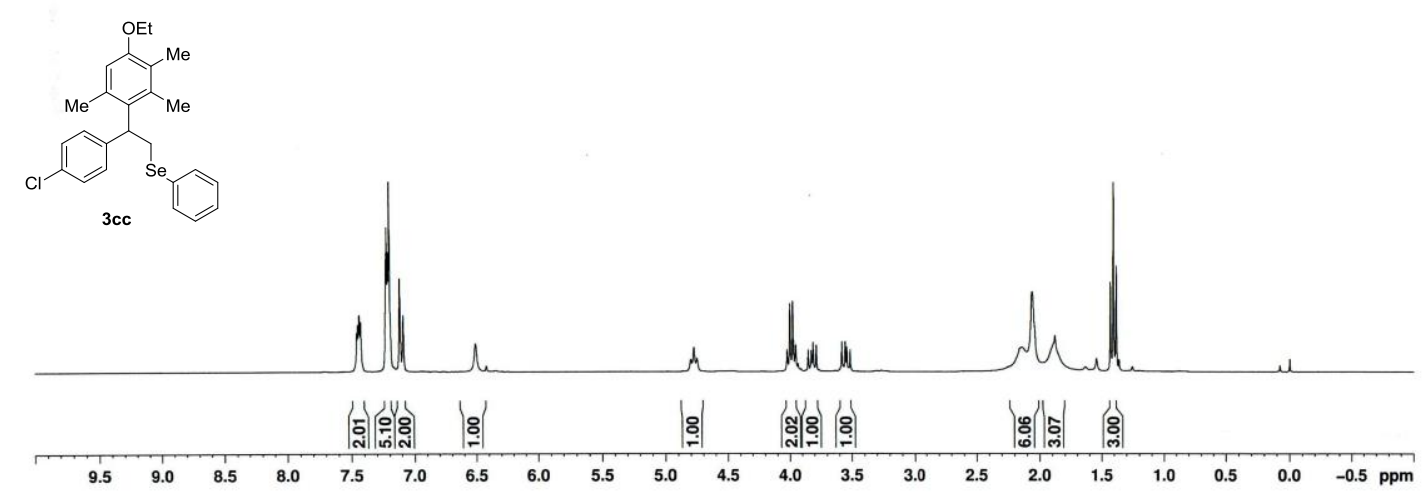

${ }^{13} \mathrm{C}$ NMR $\left(75 \mathrm{MHz}, \mathrm{CDCl}_{3}\right)$

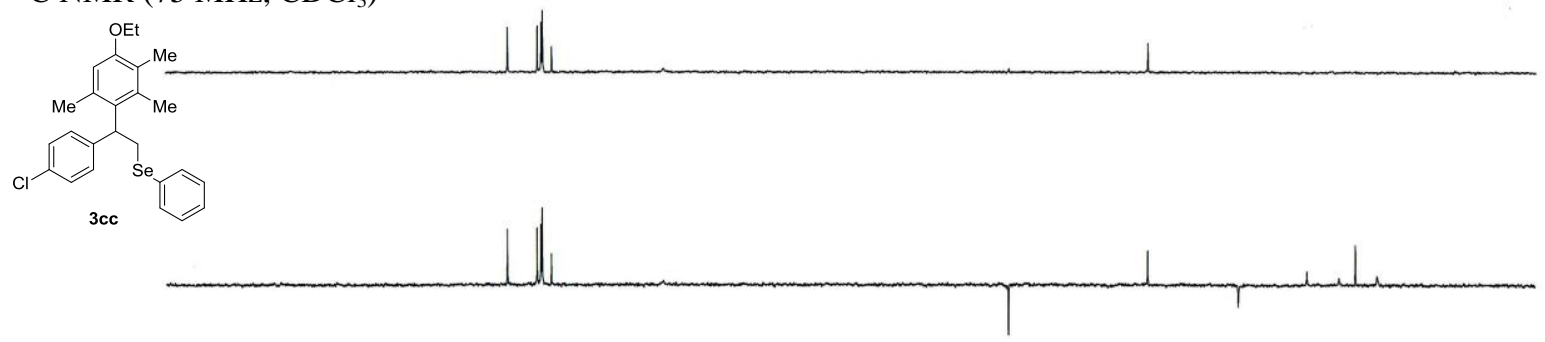

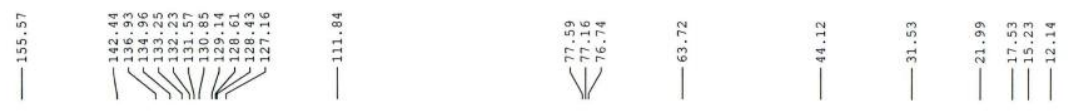

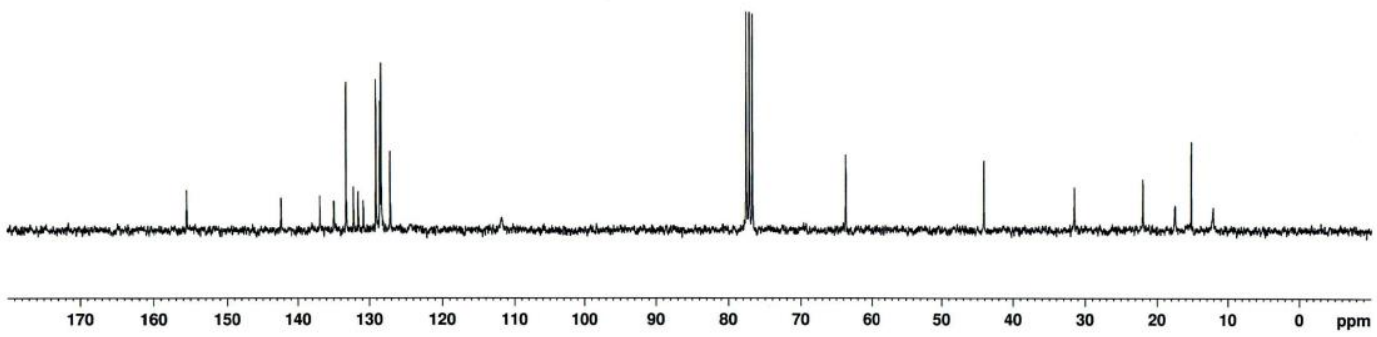



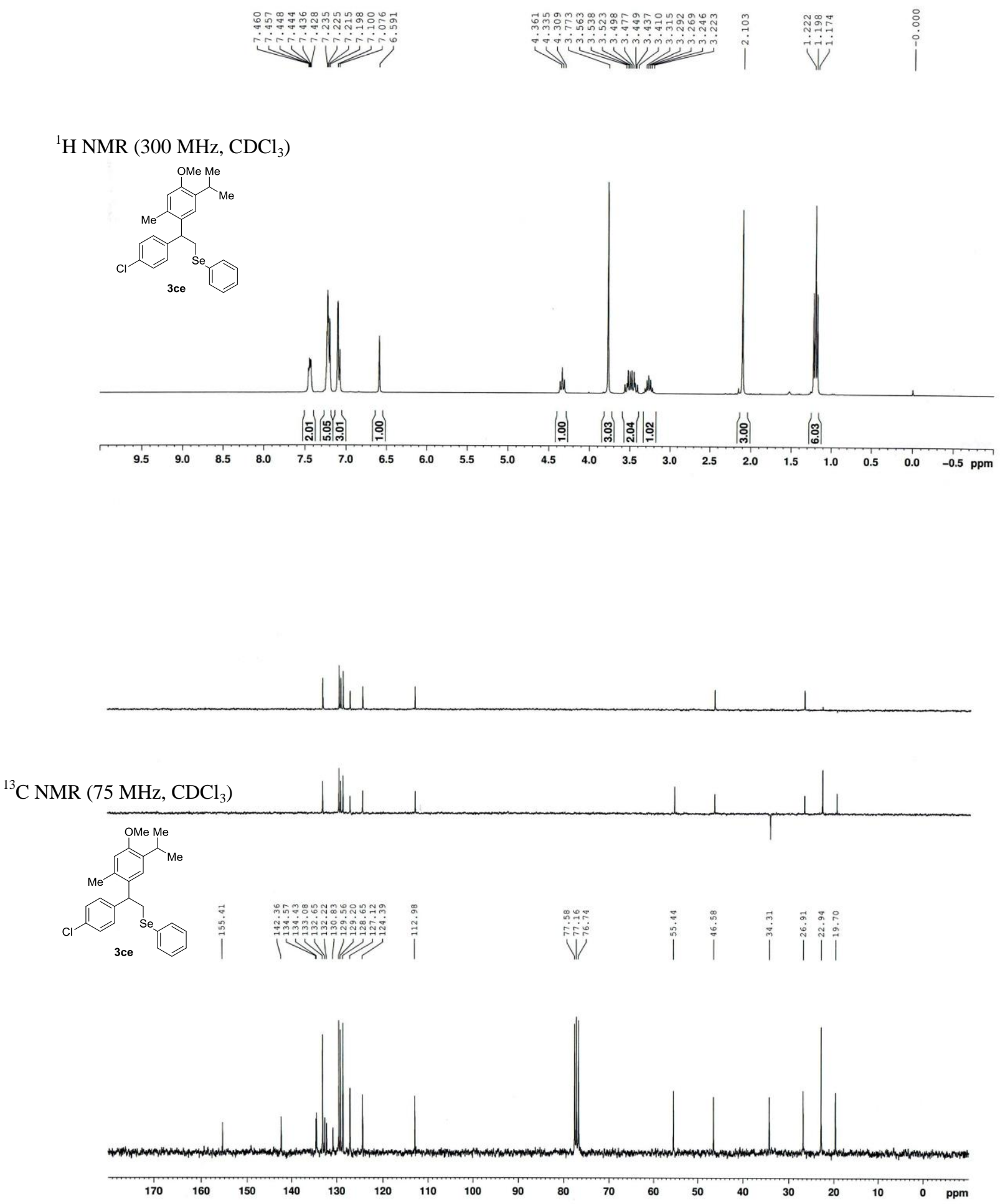


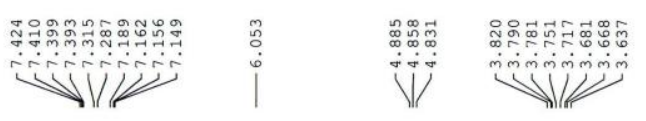

$\vdots$
$\vdots$
$\dot{1}$

${ }^{1} \mathrm{H}$ NMR (300 MHz, $\mathrm{CDCl}_{3}$ )

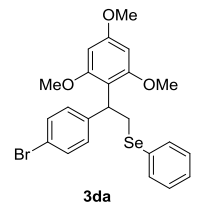

3da
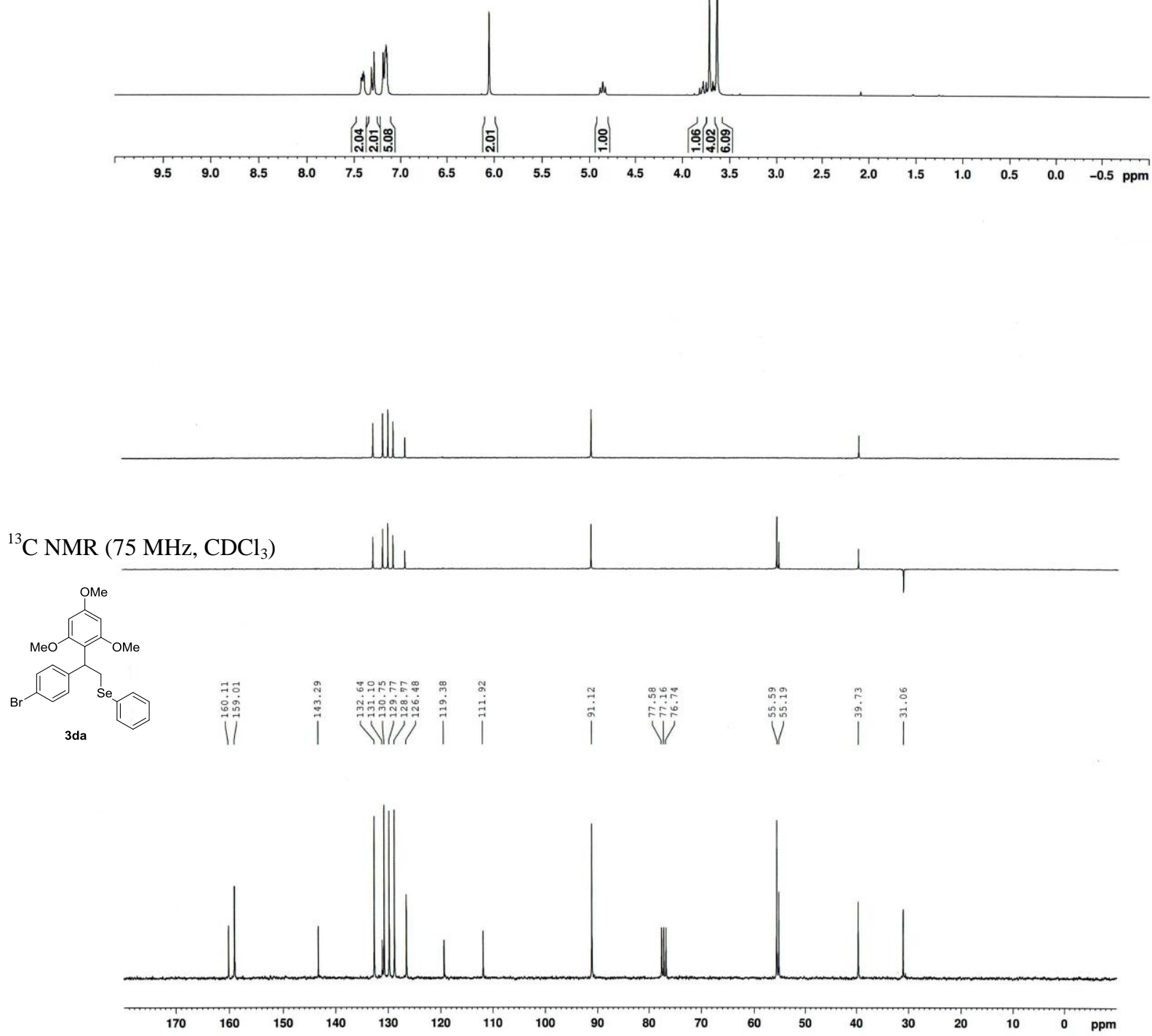

S51 
${ }^{1} \mathrm{H}$ NMR (300 MHz, $\mathrm{CDCl}_{3}$ )
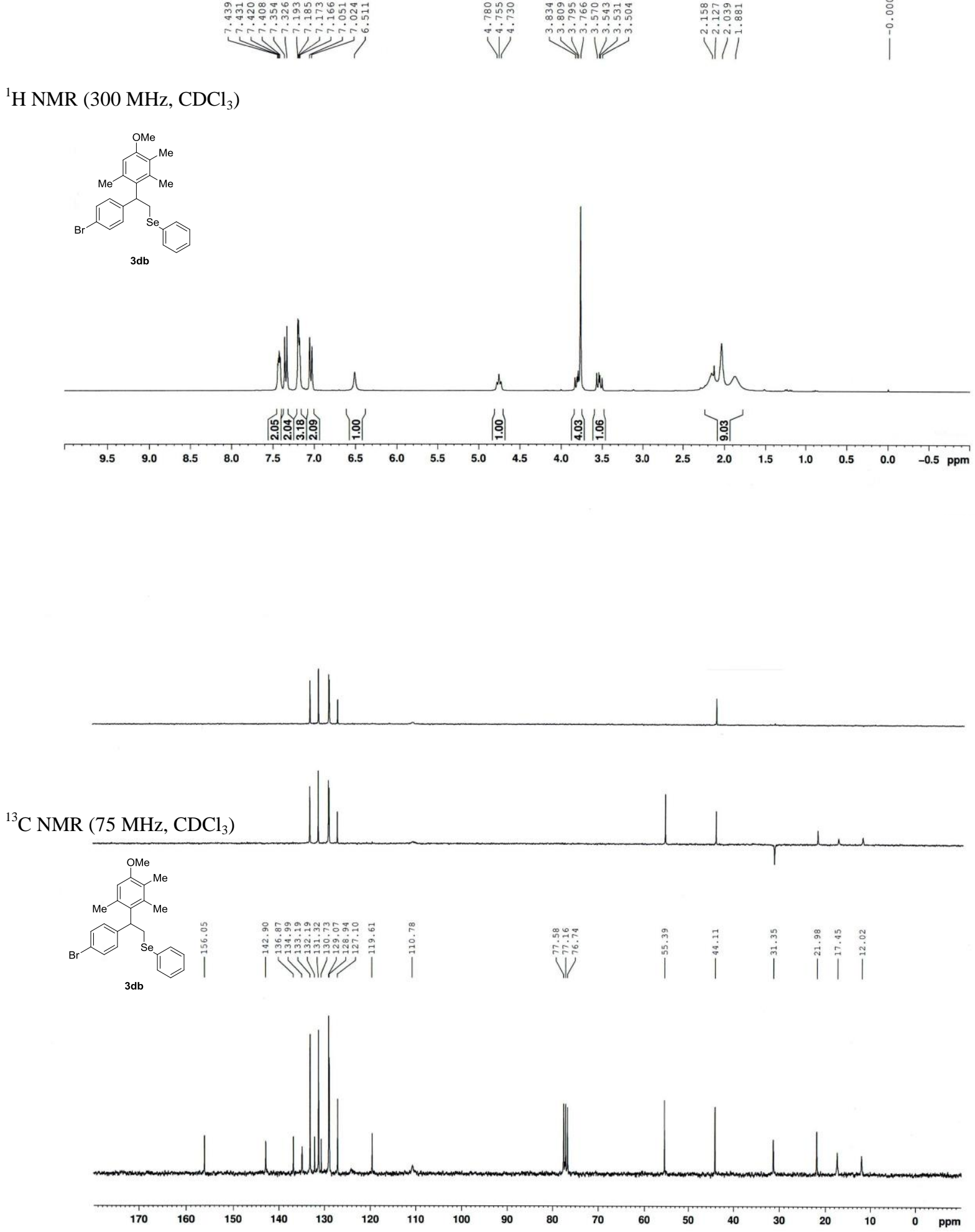


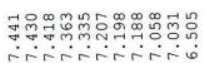

${ }^{1} \mathrm{H}$ NMR (300 MHz, $\mathrm{CDCl}_{3}$ )
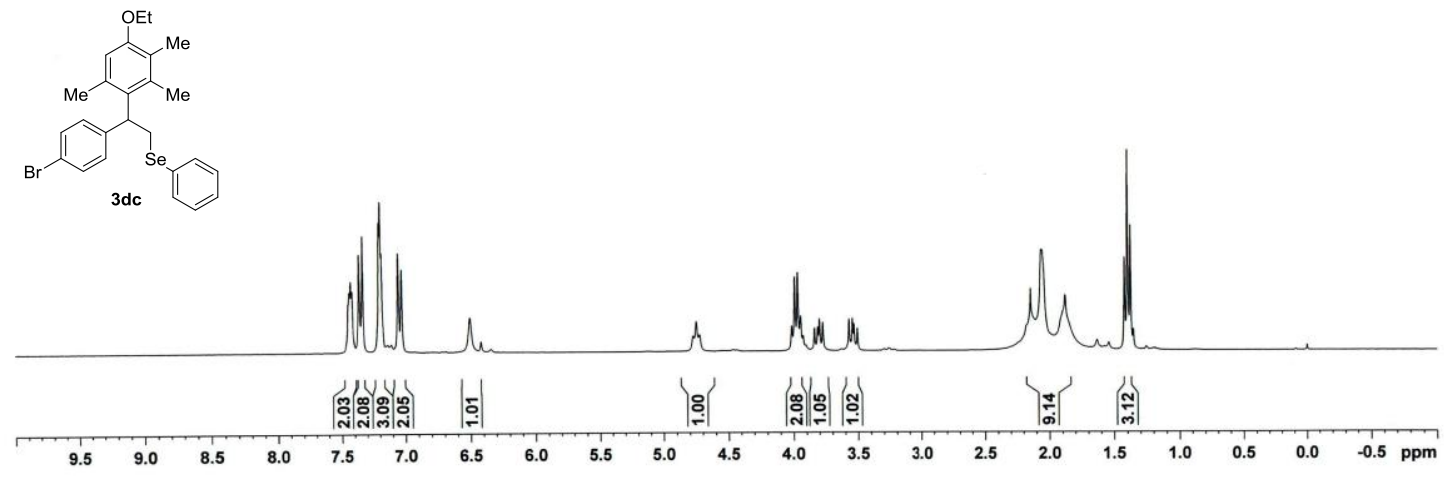

${ }^{13} \mathrm{C}$ NMR $\left(75 \mathrm{MHz}, \mathrm{CDCl}_{3}\right)$
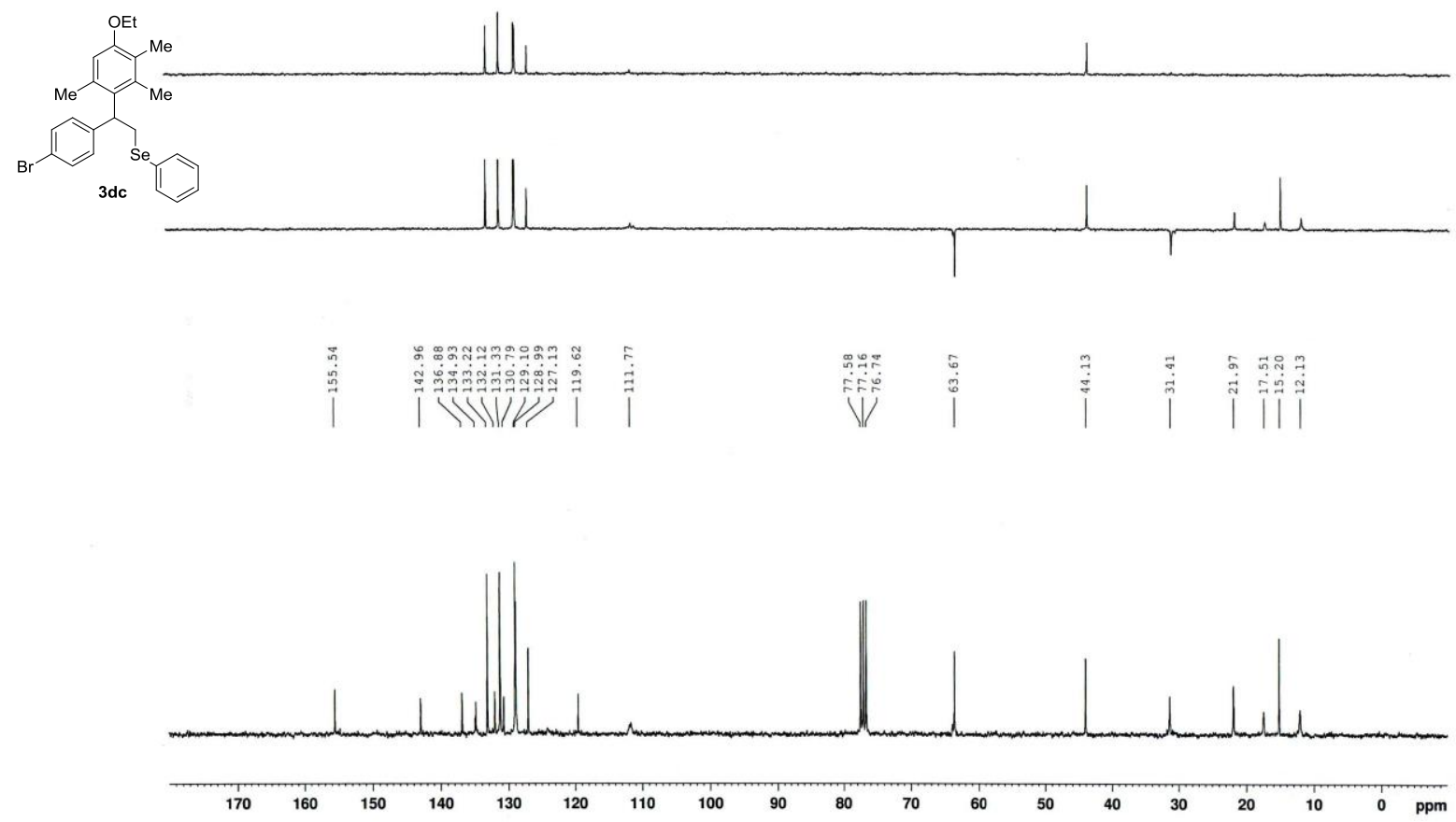

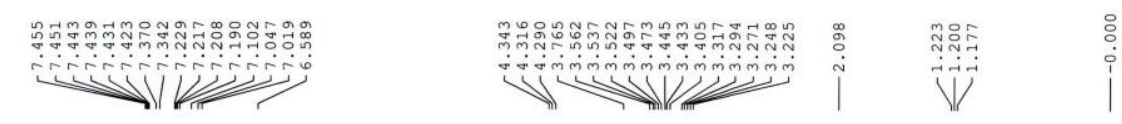

${ }^{1} \mathrm{H}$ NMR (300 MHz, $\mathrm{CDCl}_{3}$ )
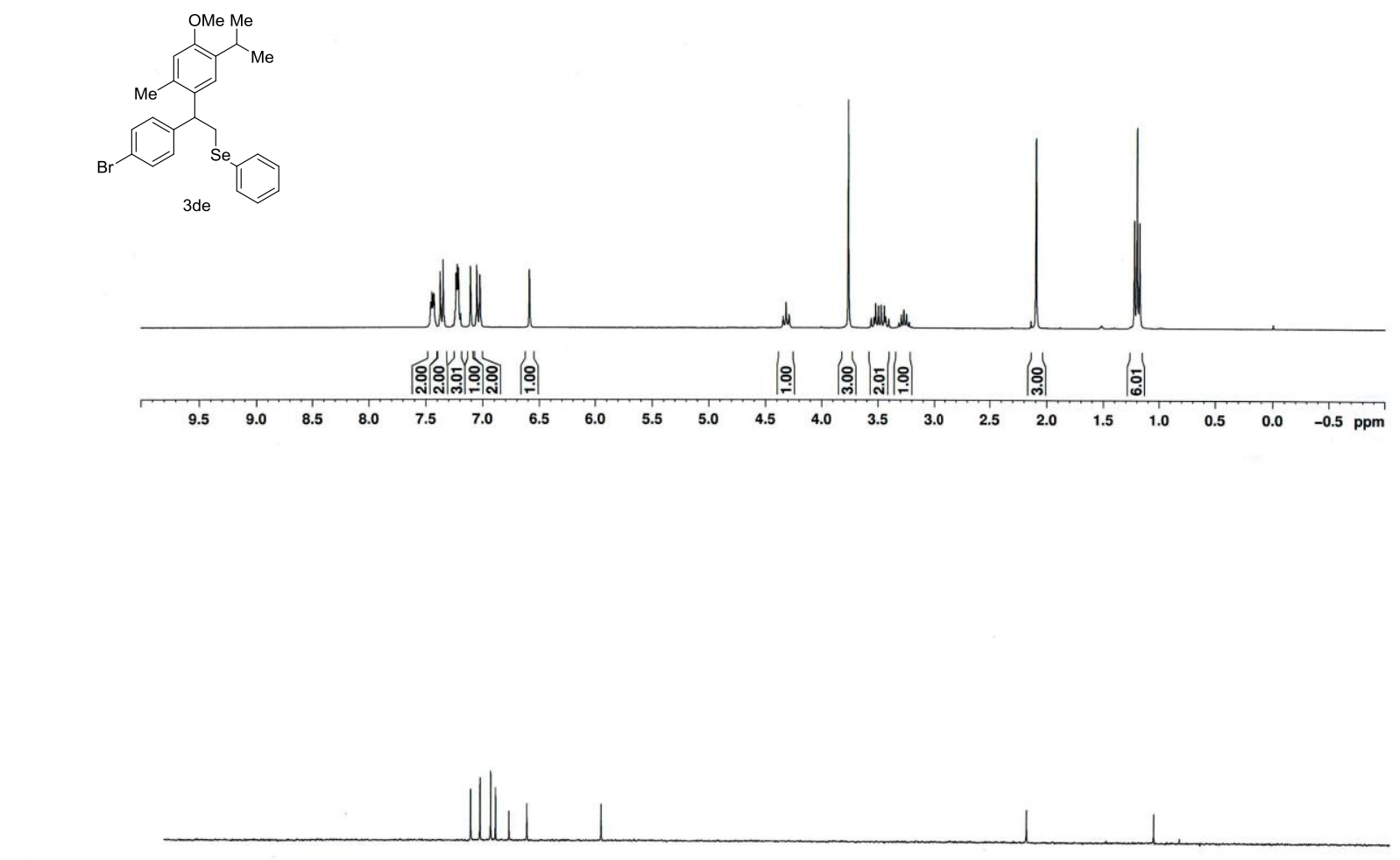

${ }^{13} \mathrm{C}$ NMR (75 MHz, $\mathrm{CDCl}_{3}$ )
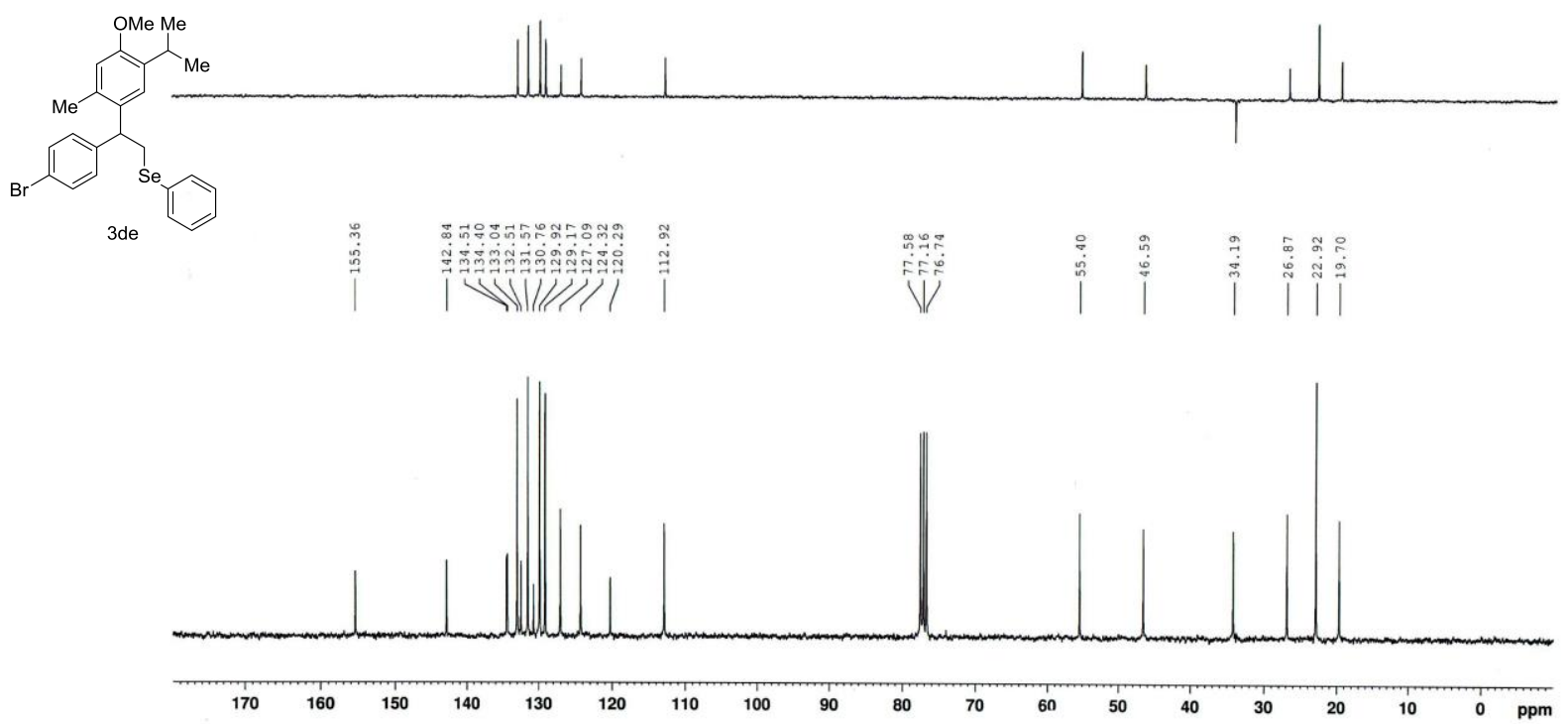


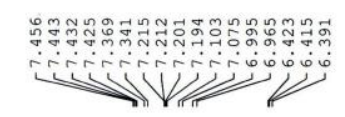

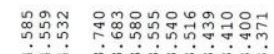

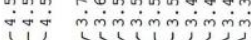

${ }^{1} \mathrm{H}$ NMR (300 MHz, $\mathrm{CDCl}_{3}$ )
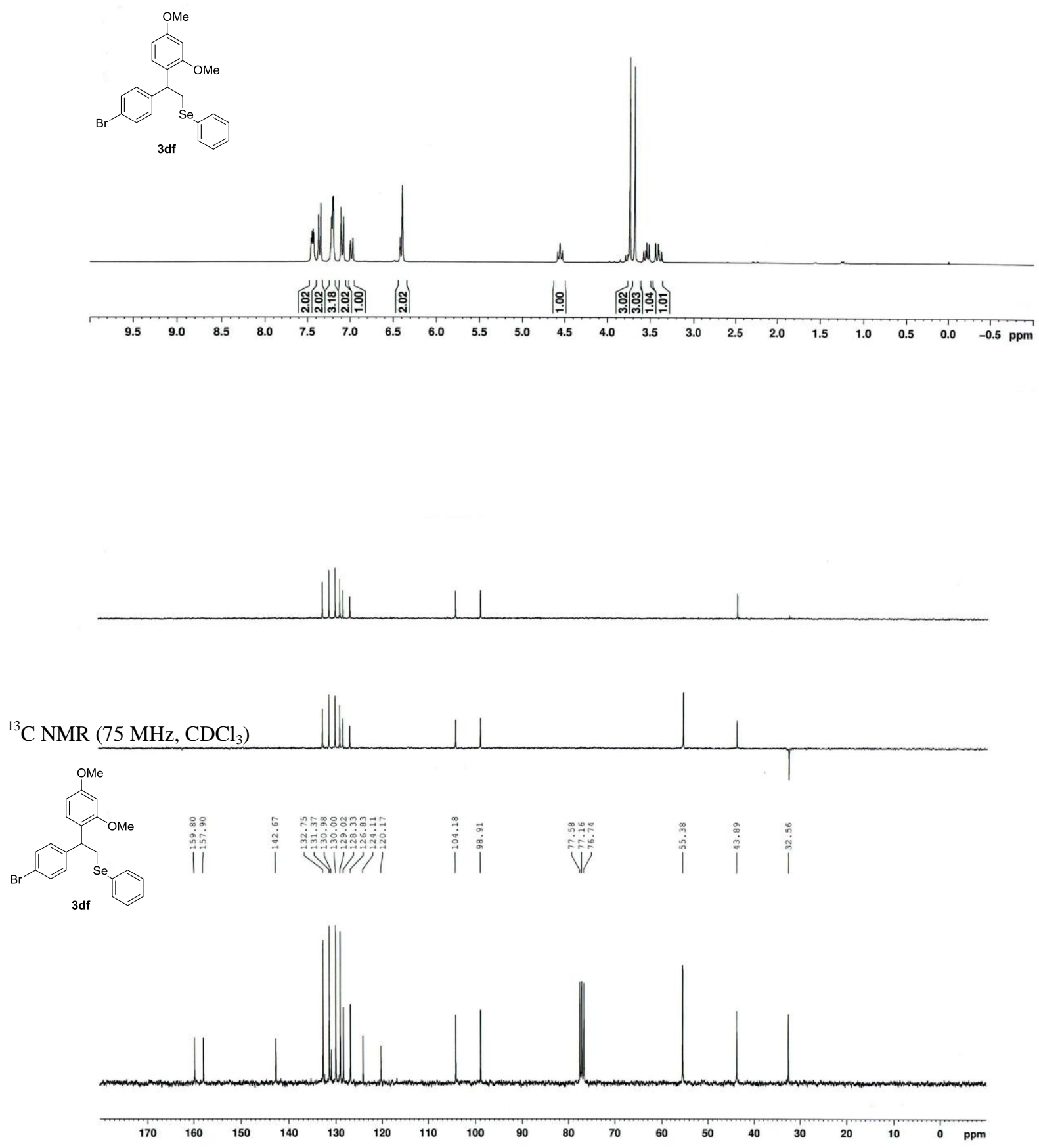


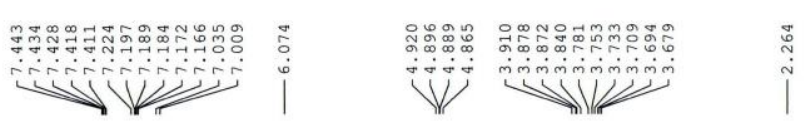

0
$\vdots$
$\vdots$

${ }^{1} \mathrm{H}$ NMR $\left(300 \mathrm{MHz}, \mathrm{CDCl}_{3}\right.$ )
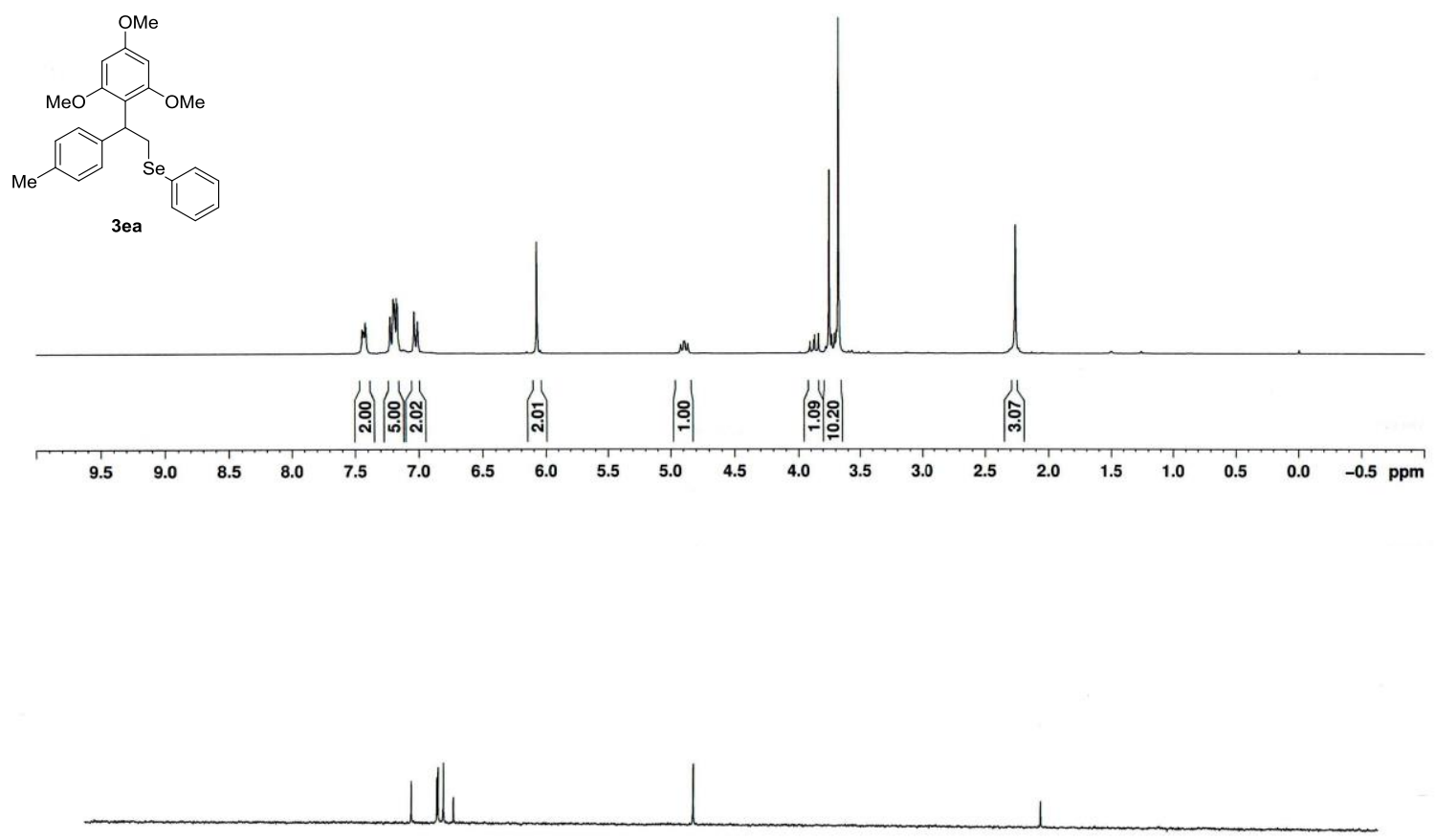

${ }^{13} \mathrm{C}$ NMR $\left(75 \mathrm{MHz}, \mathrm{CDCl}_{3}\right)$

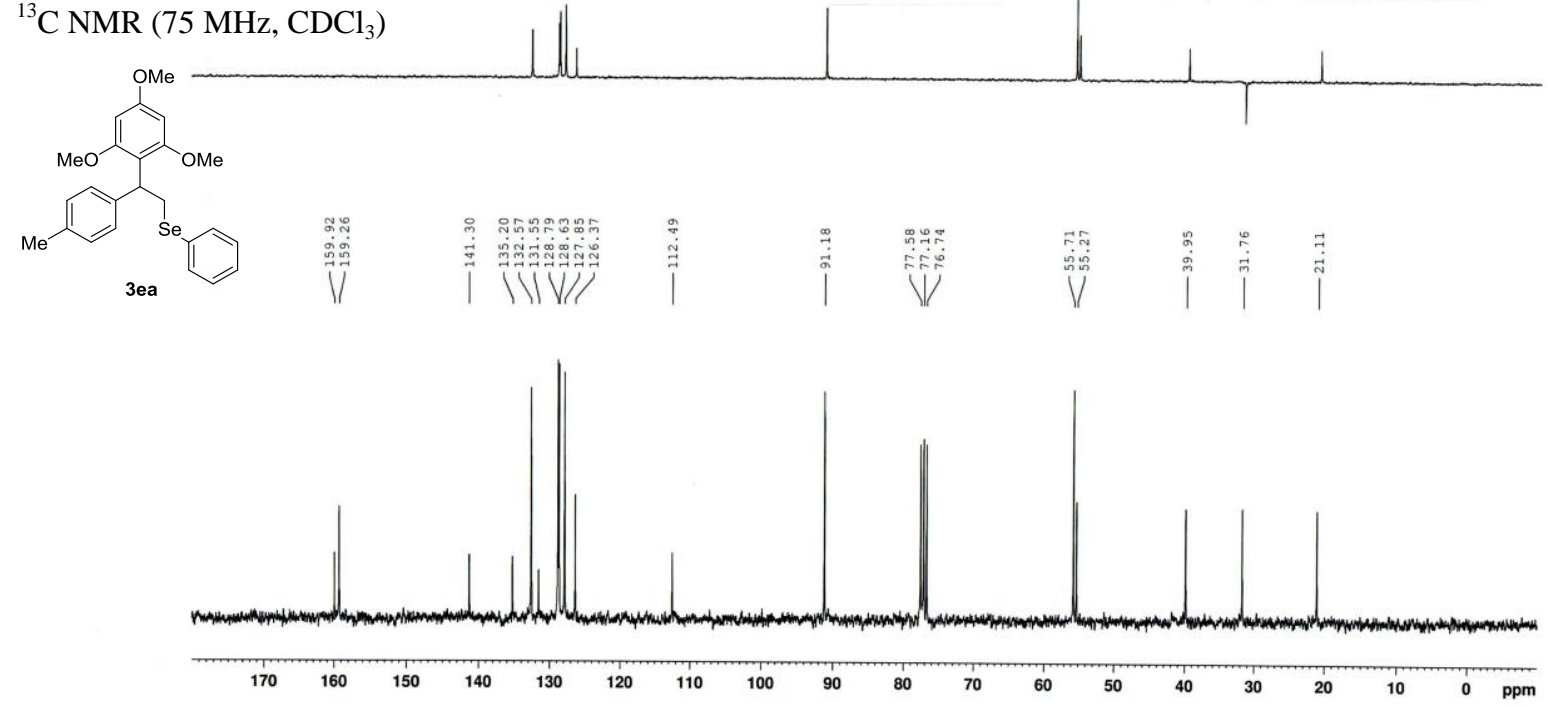

S56 


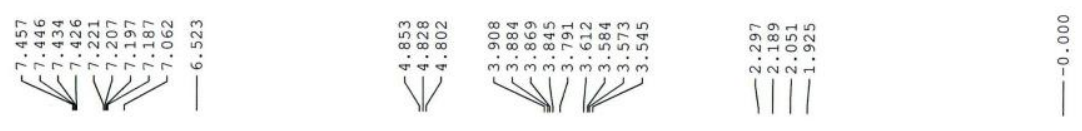

${ }^{1} \mathrm{H}$ NMR (300 MHz, $\mathrm{CDCl}_{3}$ )

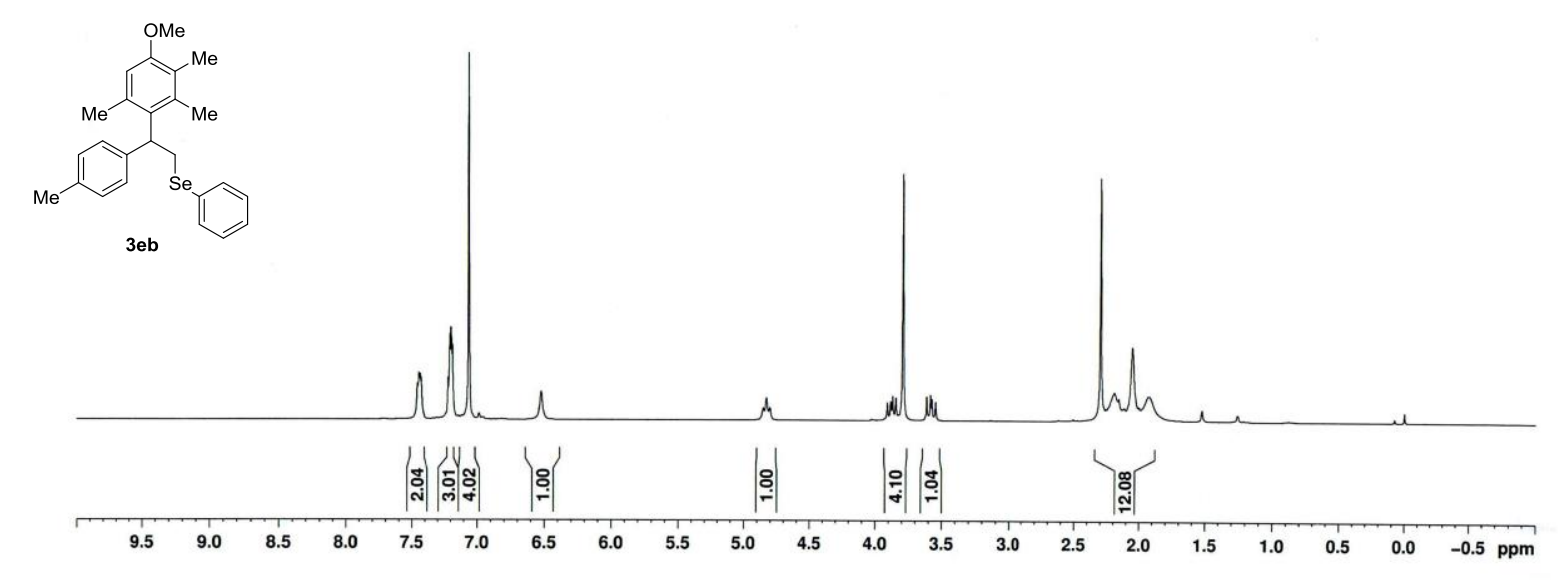

V|

\section{NMR (300 MHz, $\left.\mathrm{CDCl}_{3}\right)$}
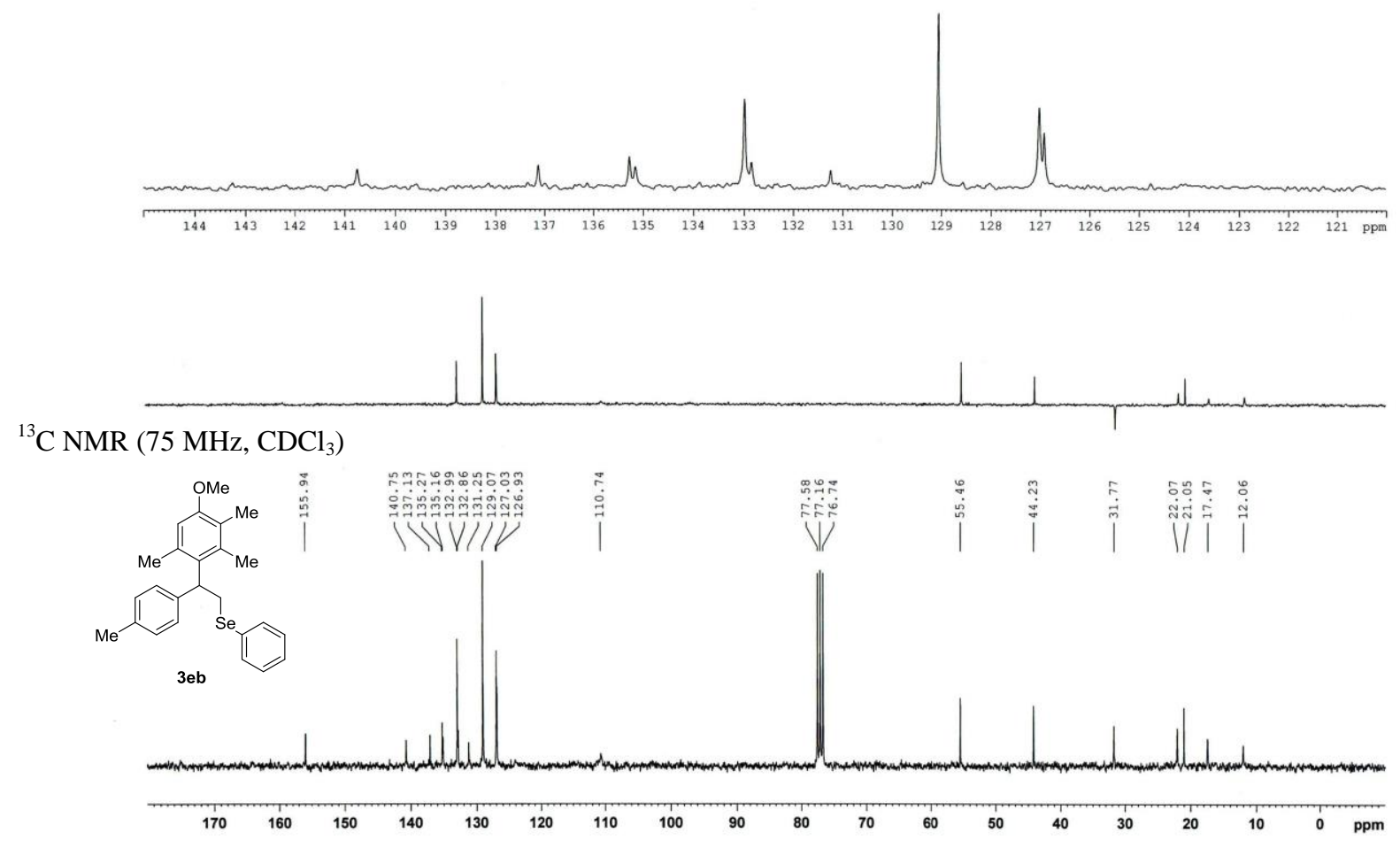
${ }^{1} \mathrm{H}$ NMR $\left(300 \mathrm{MHz}, \mathrm{CDCl}_{3}\right)$
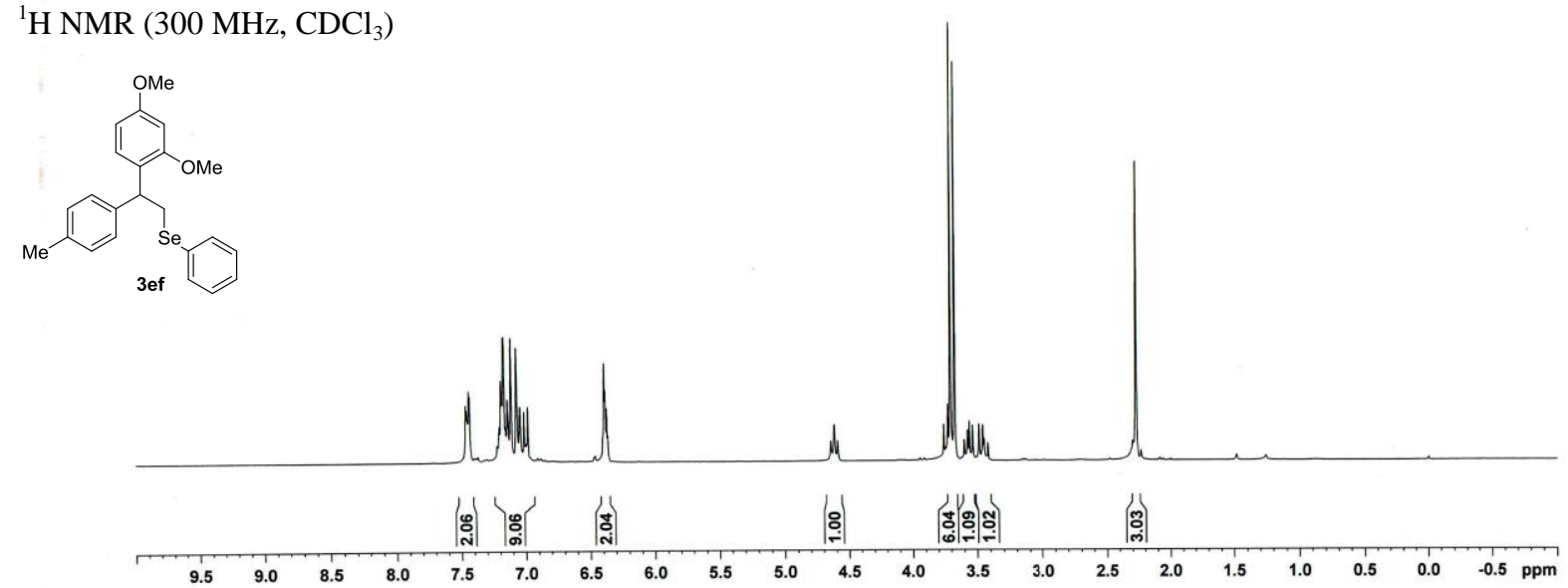

${ }^{13} \mathrm{C}$ NMR $\left(75 \mathrm{MHz}, \mathrm{CDCl}_{3}\right.$ )

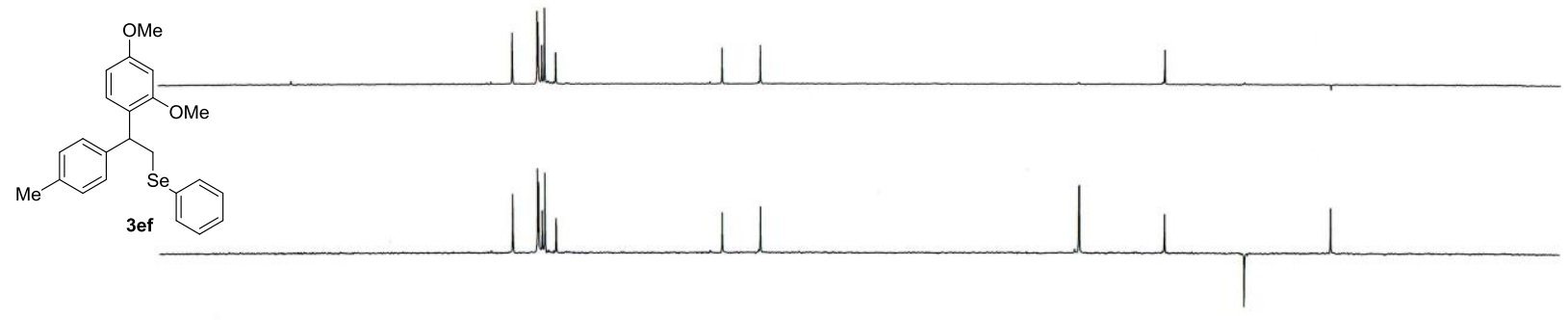

||

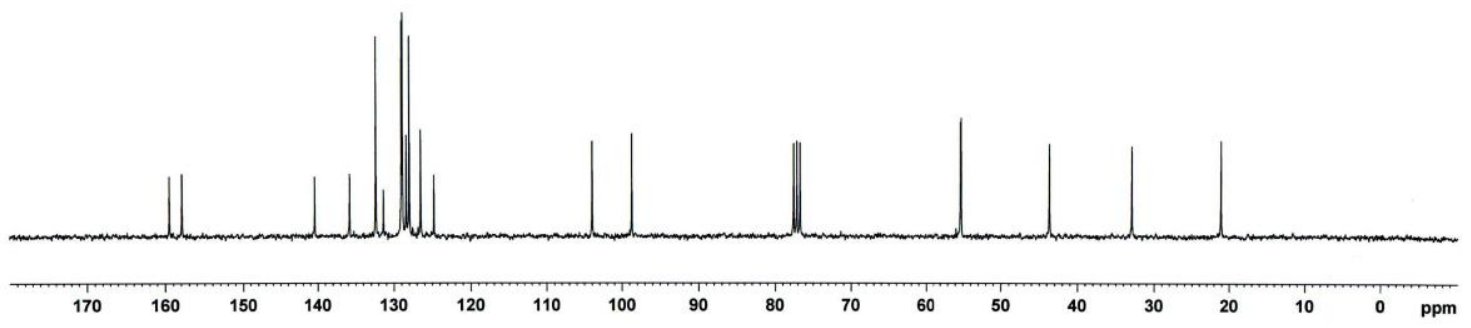




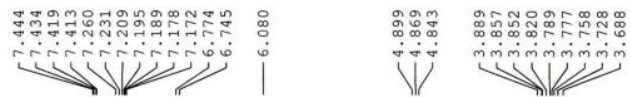

${ }^{1} \mathrm{H}$ NMR (300 MHz, $\mathrm{CDCl}_{3}$ )
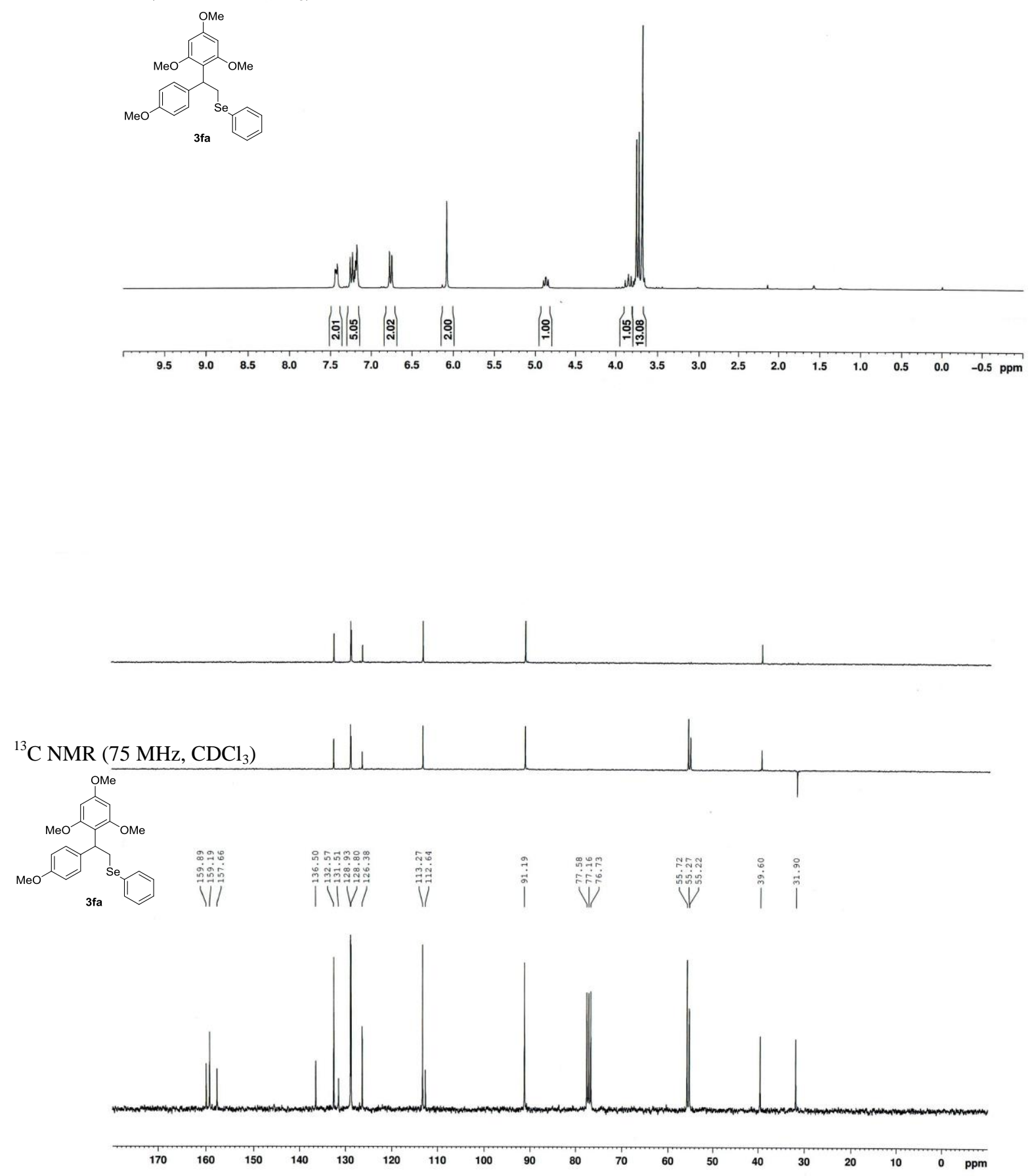

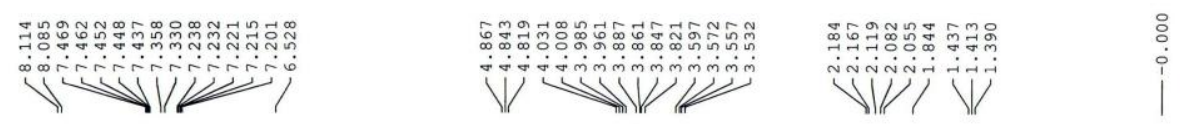

${ }^{1} \mathrm{H}$ NMR (300 MHz, $\mathrm{CDCl}_{3}$ )
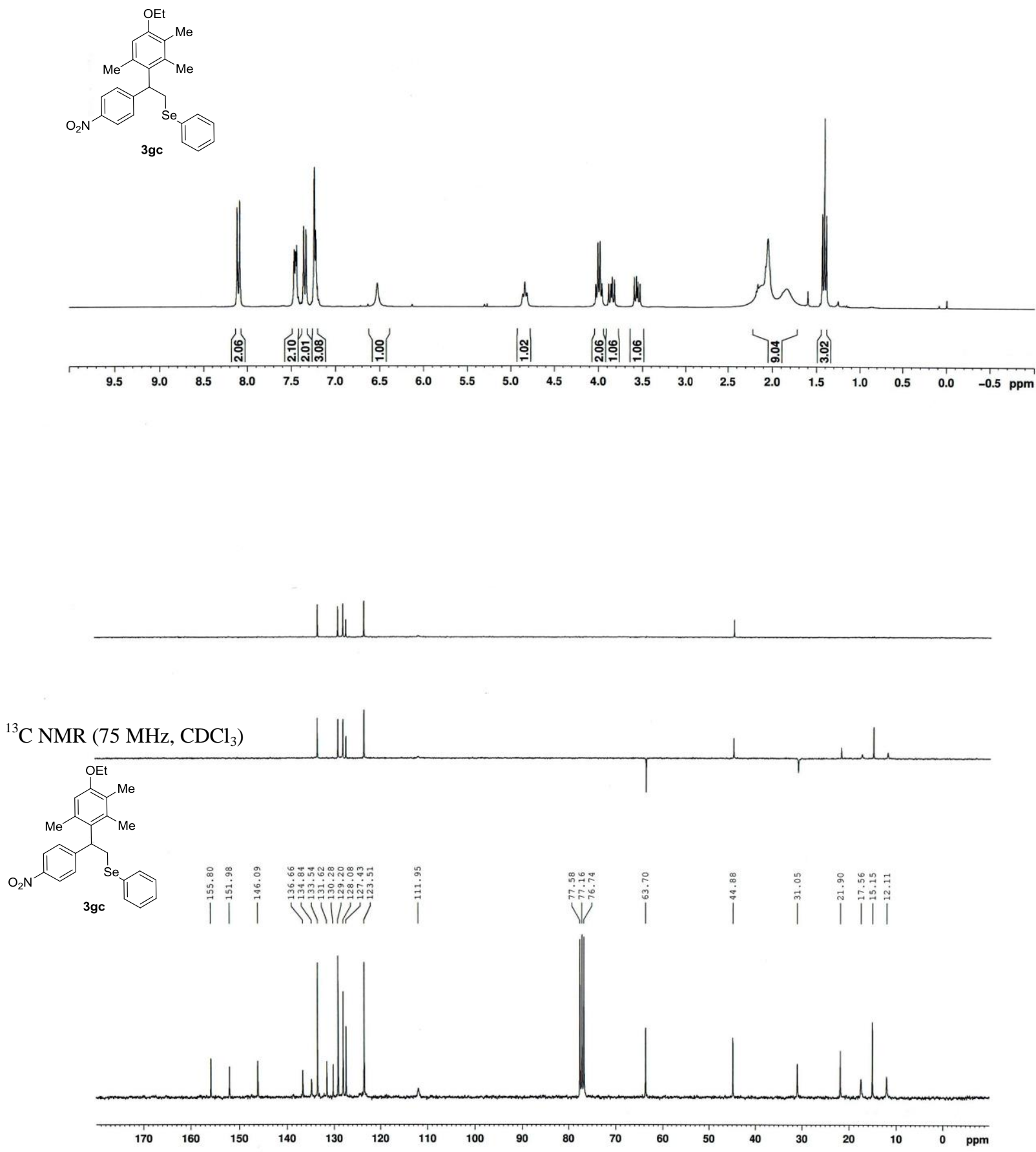


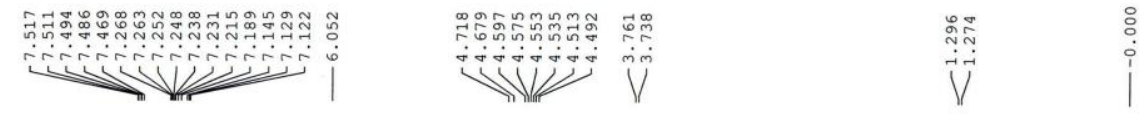

${ }^{1} \mathrm{H}$ NMR $\left(300 \mathrm{MHz}, \mathrm{CDCl}_{3}\right.$ )

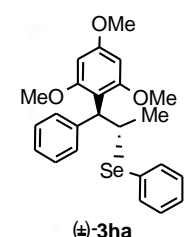

( $)$-3ha
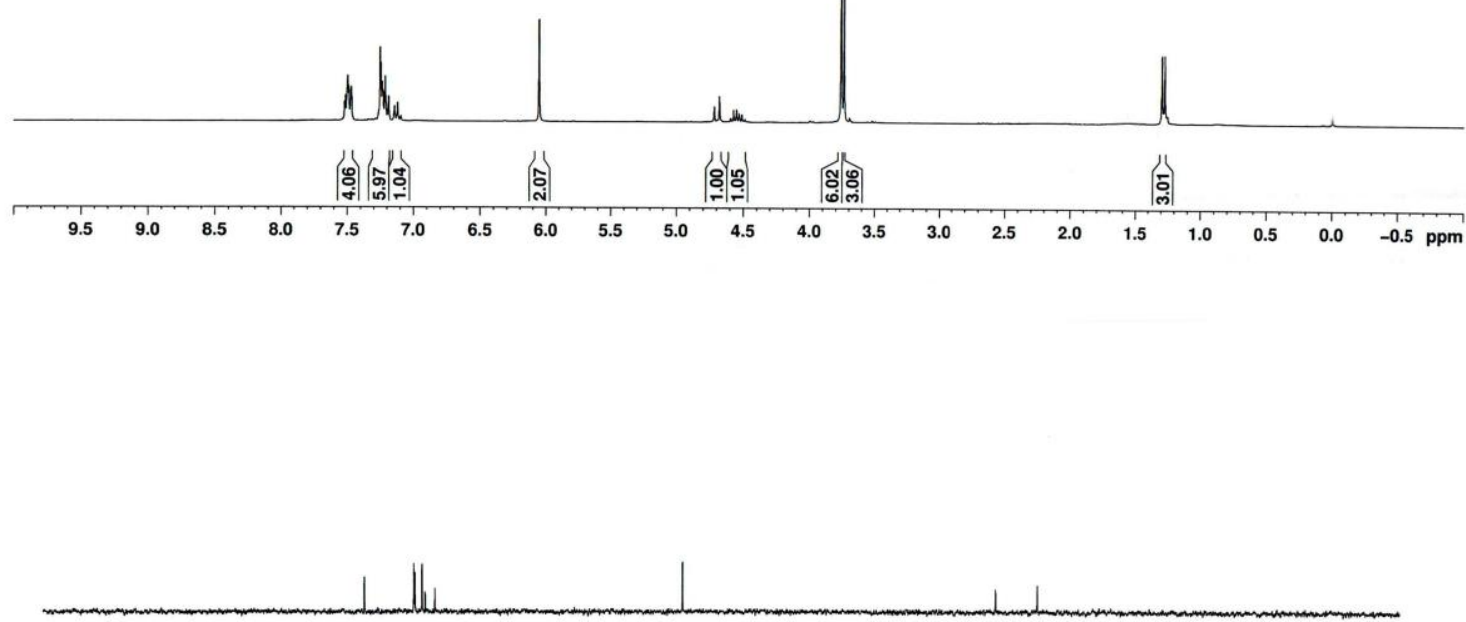

${ }^{13} \mathrm{C}$ NMR $\left(75 \mathrm{MHz}, \mathrm{CDCl}_{3}\right)$

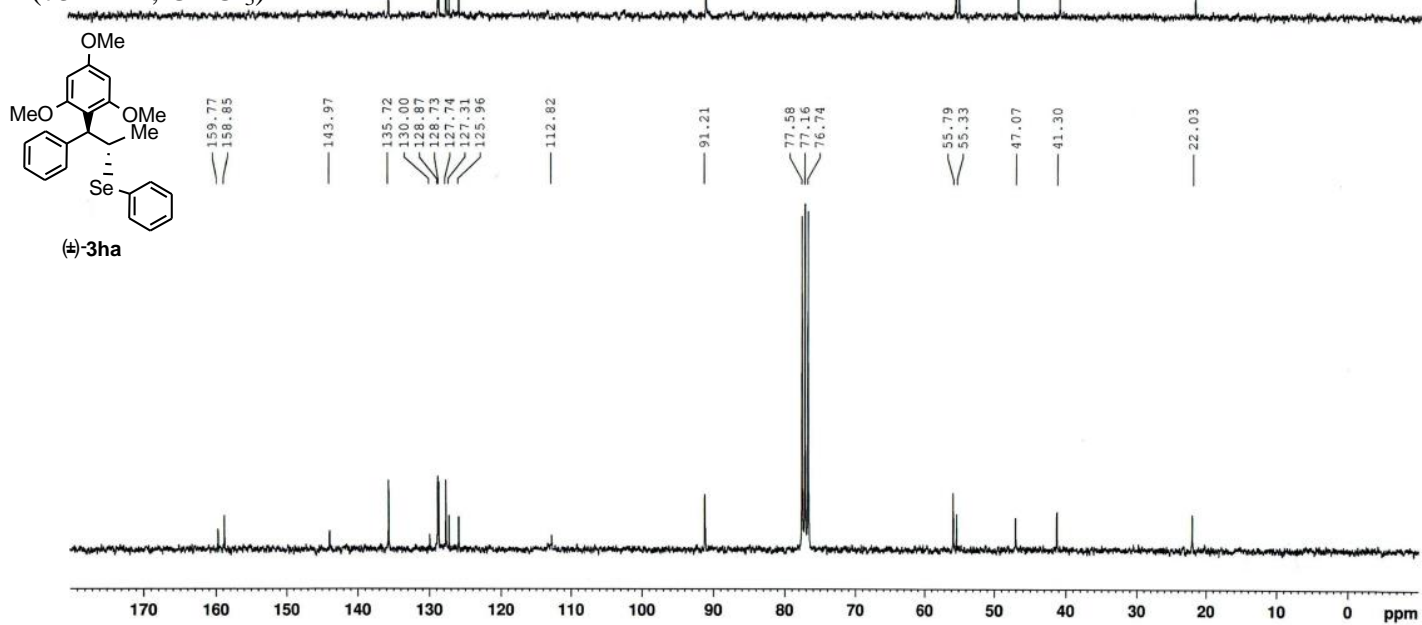




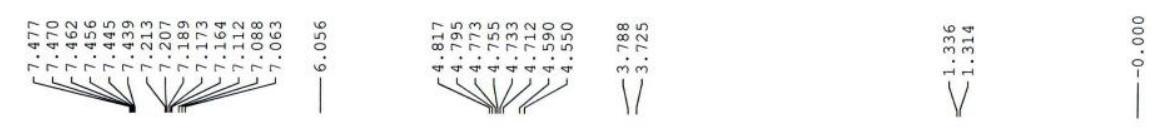

${ }^{1} \mathrm{H}$ NMR $\left(300 \mathrm{MHz}, \mathrm{CDCl}_{3}\right.$ )
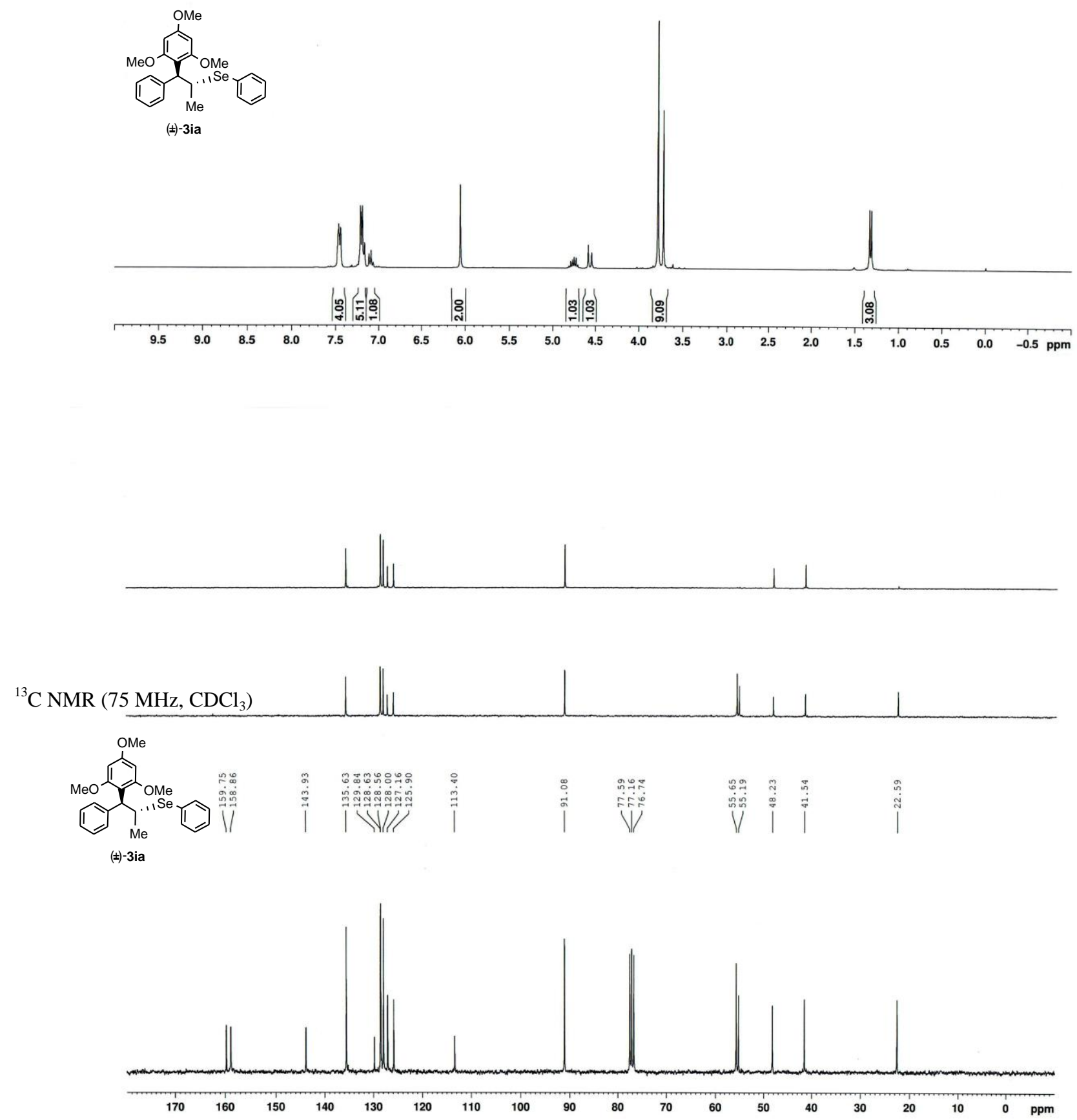


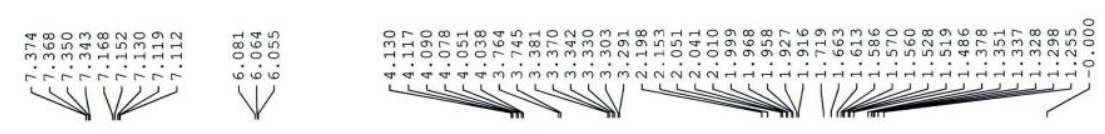

${ }^{1} \mathrm{H}$ NMR (300 MHz, $\mathrm{CDCl}_{3}$ )
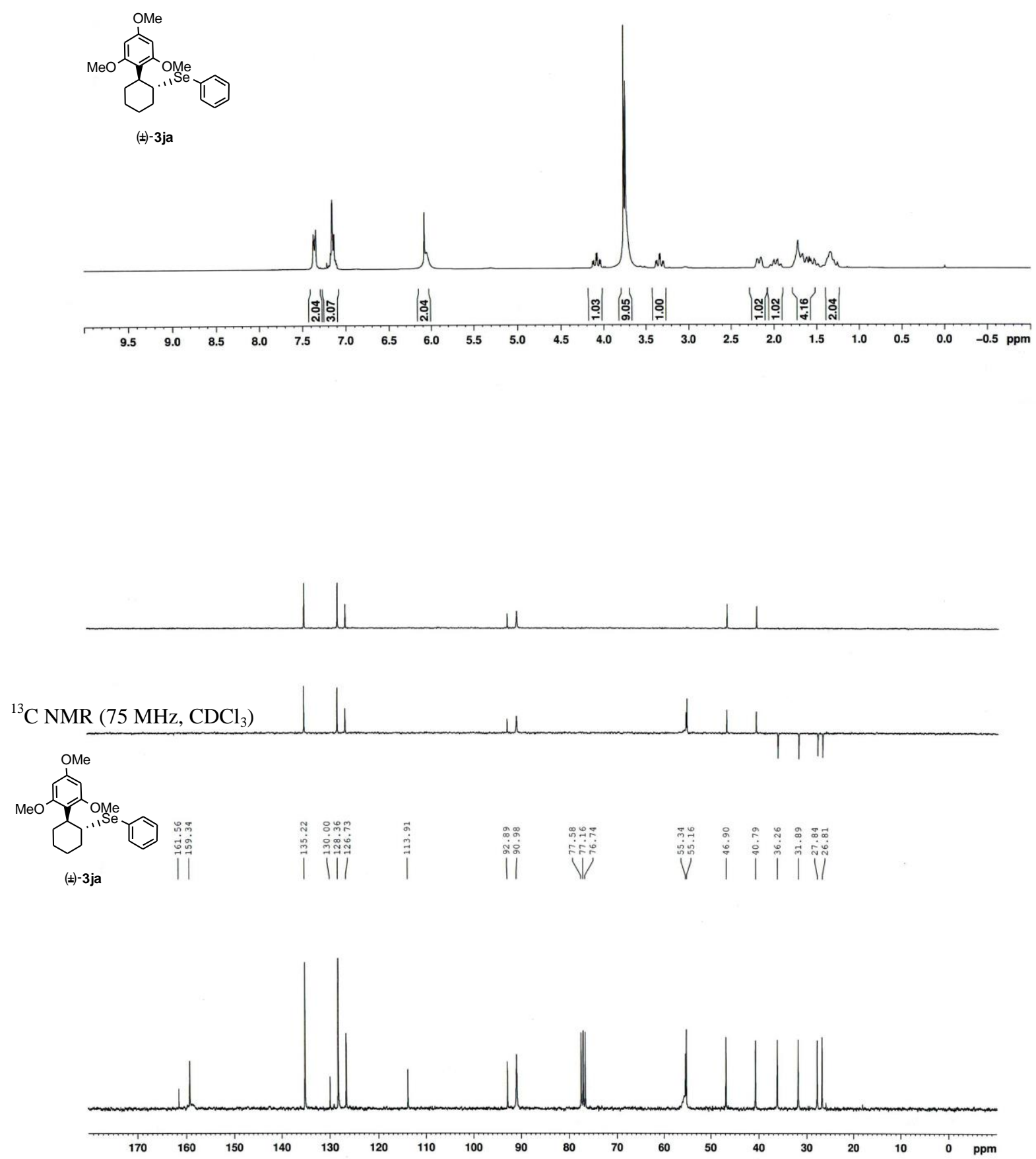

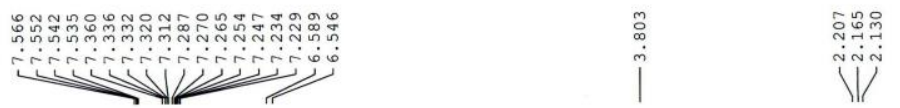

$\vdots$
$\vdots$
1

${ }^{1} \mathrm{H}$ NMR (300 MHz, $\left.\mathrm{CDCl}_{3}\right)$
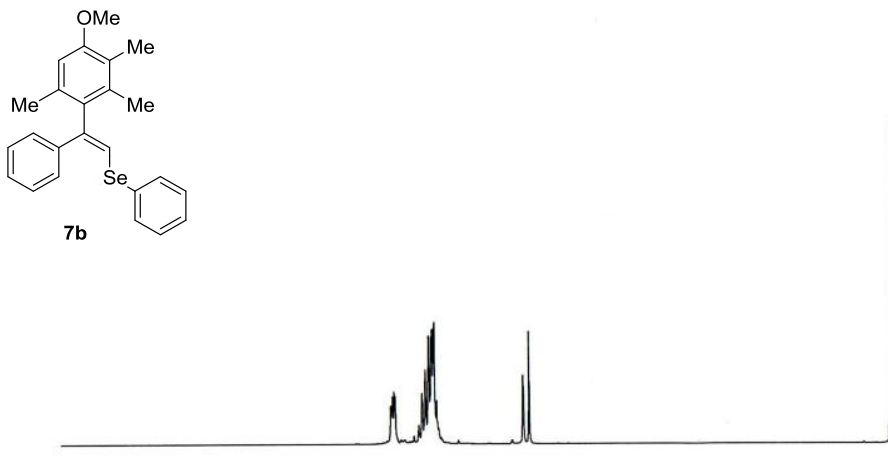

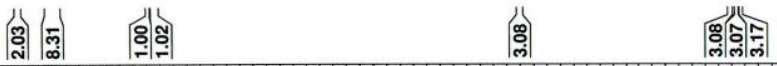

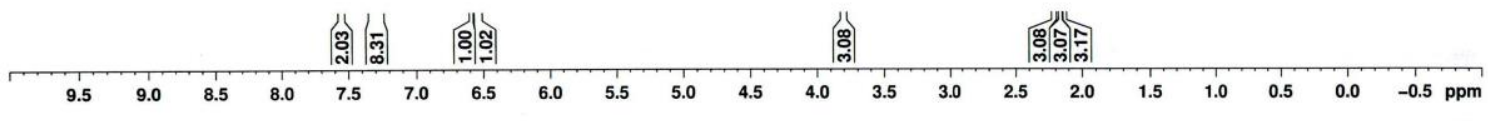

لـ
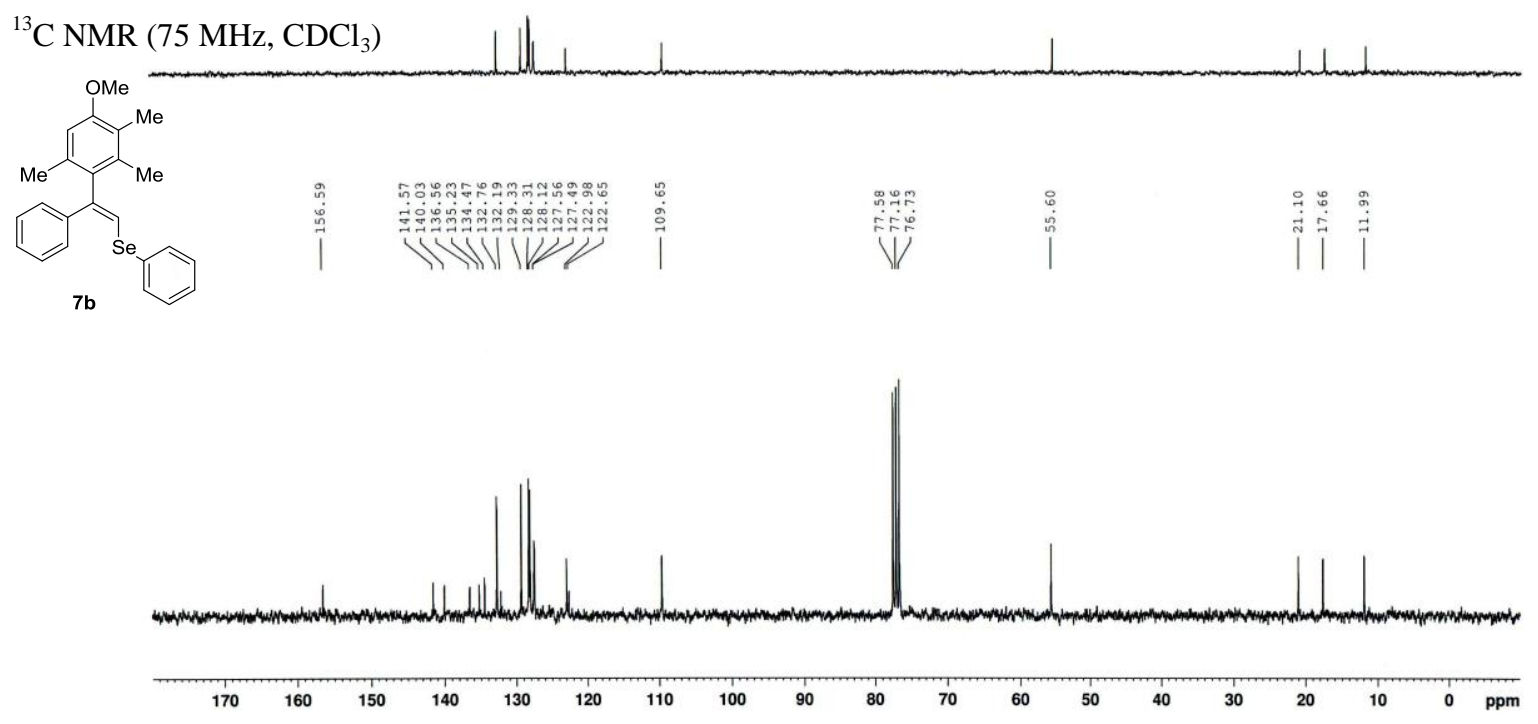
${ }^{1} \mathrm{H}$ NMR $\left(300 \mathrm{MHz}, \mathrm{CDCl}_{3}\right)$
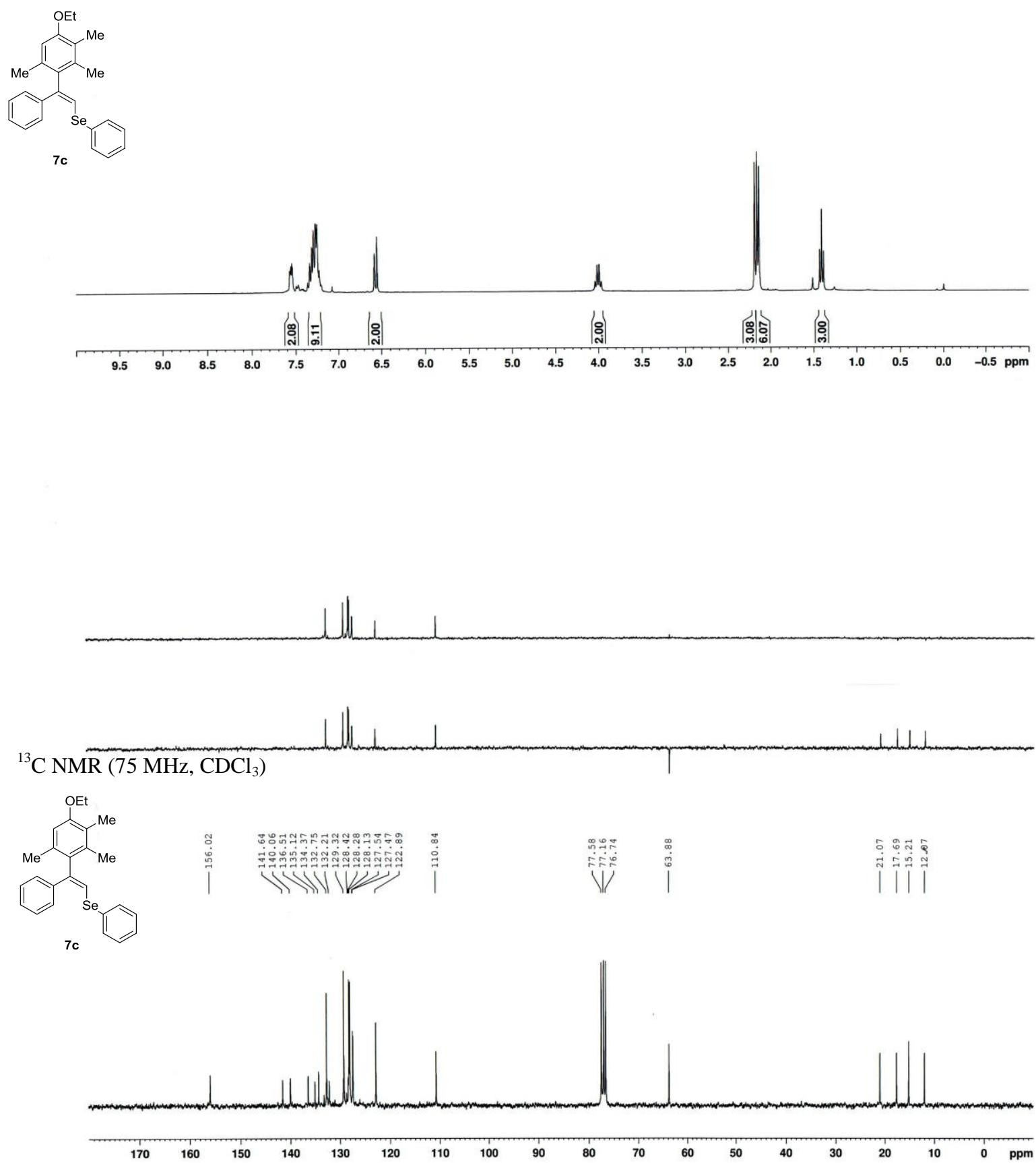


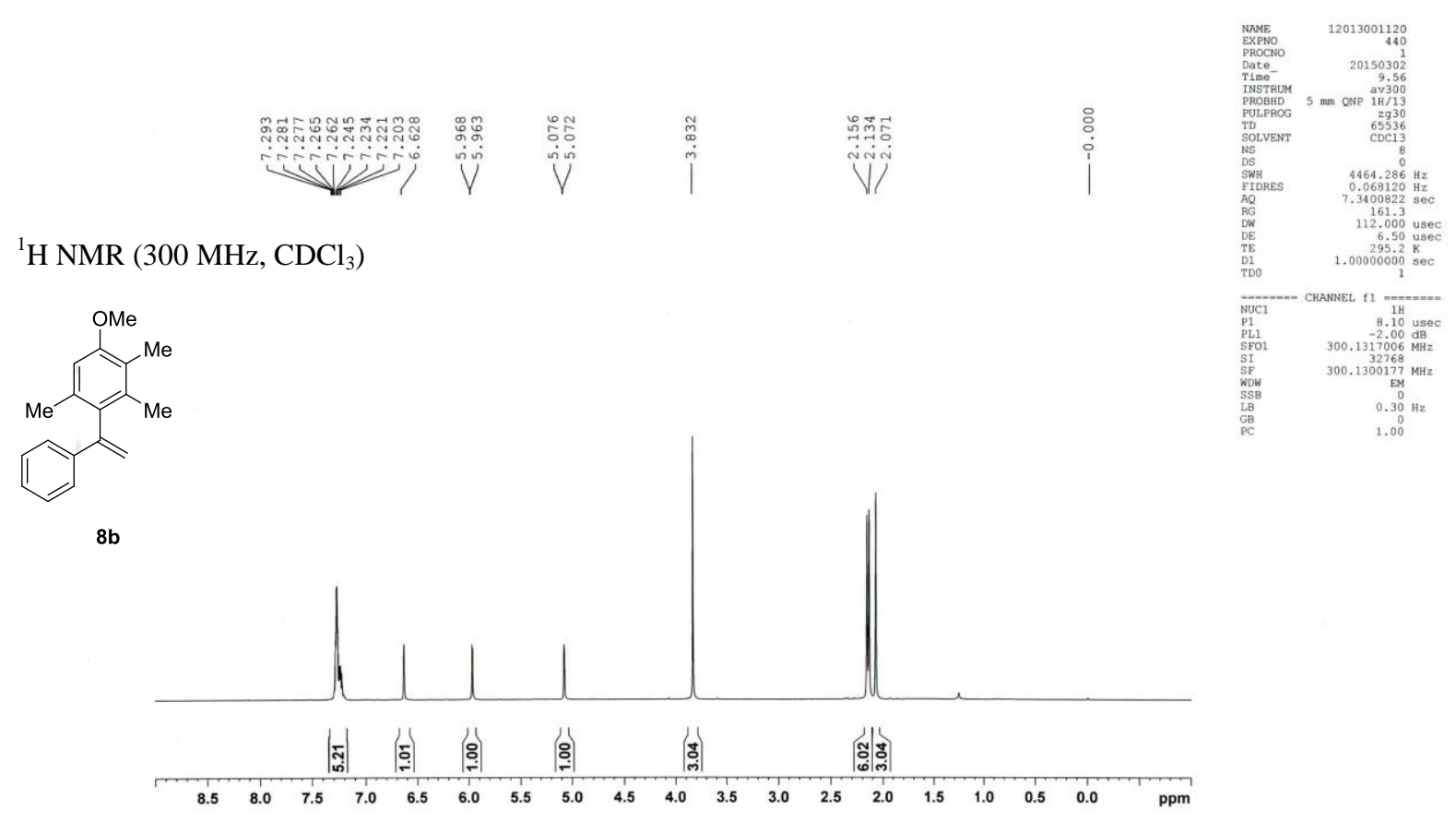

${ }^{13} \mathrm{C}$ NMR $\left(75 \mathrm{MHz}, \mathrm{CDCl}_{3}\right)$
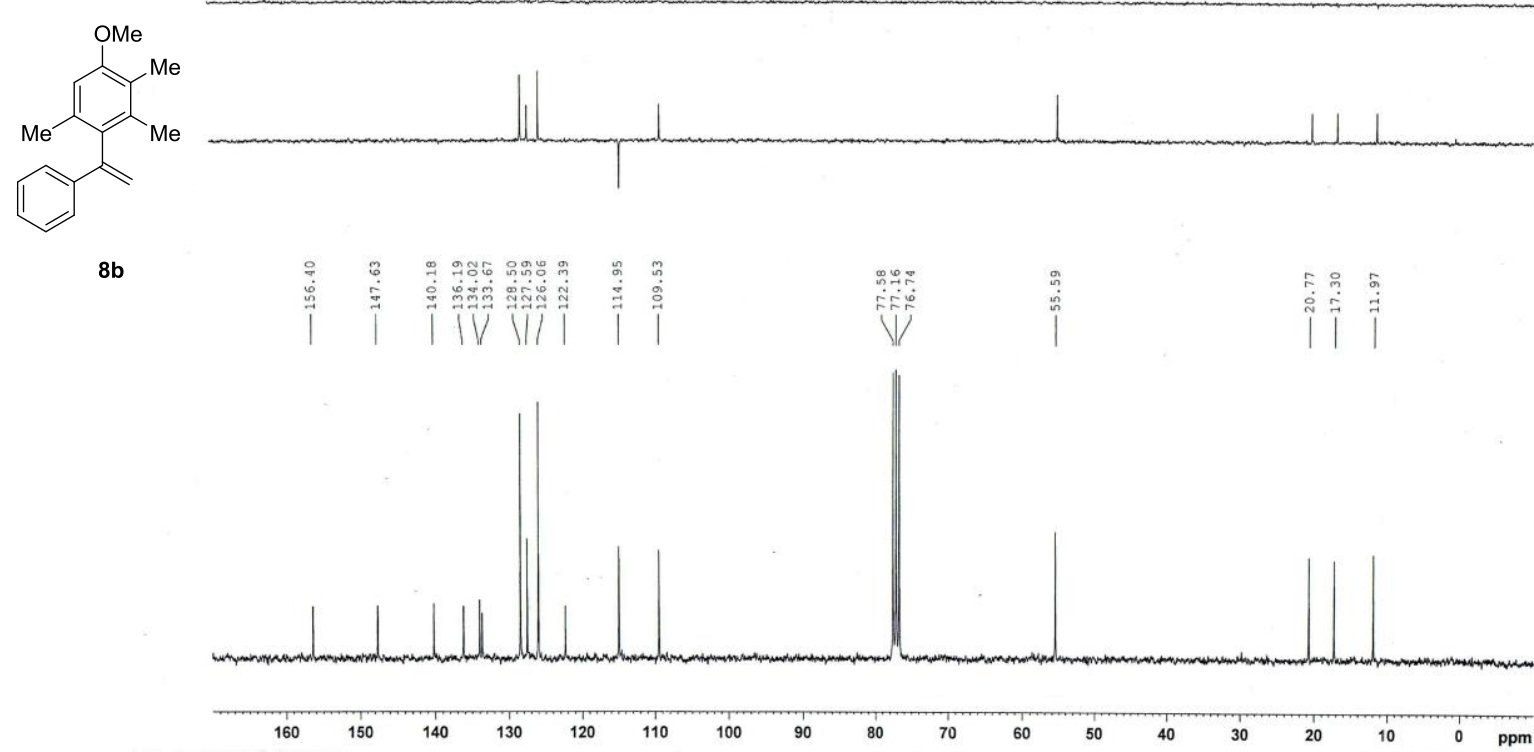

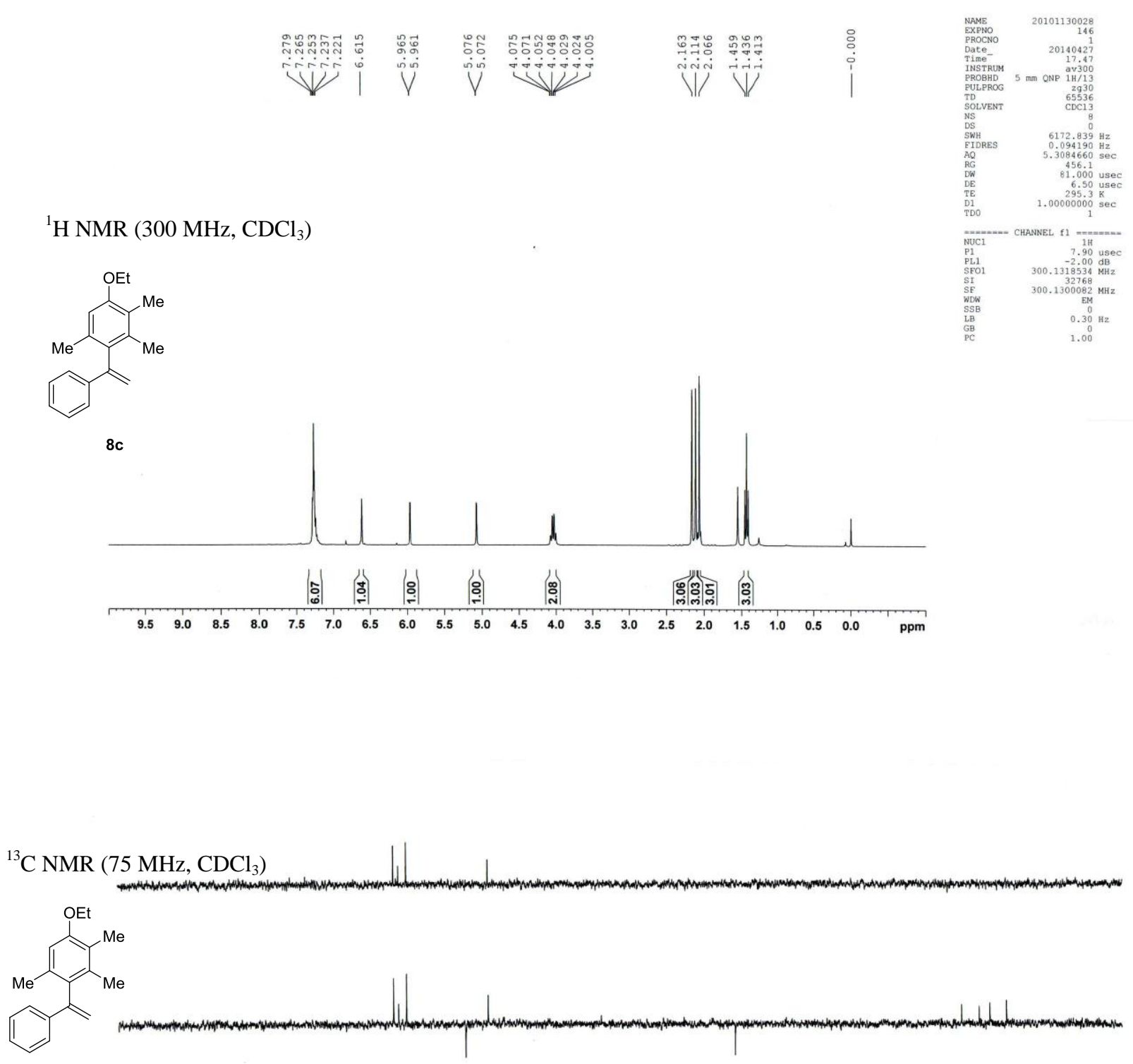

$8 c$

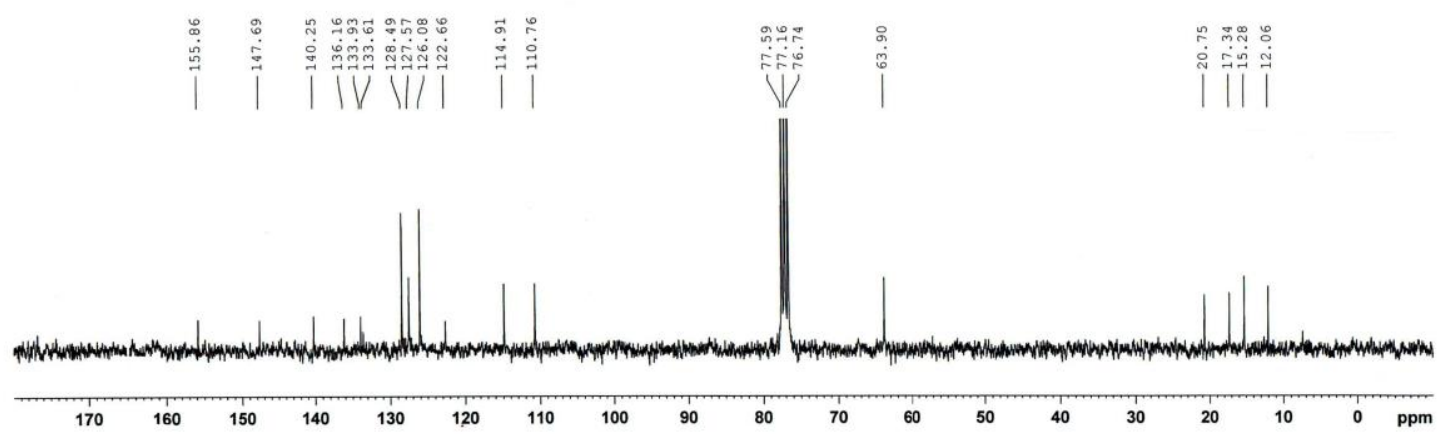



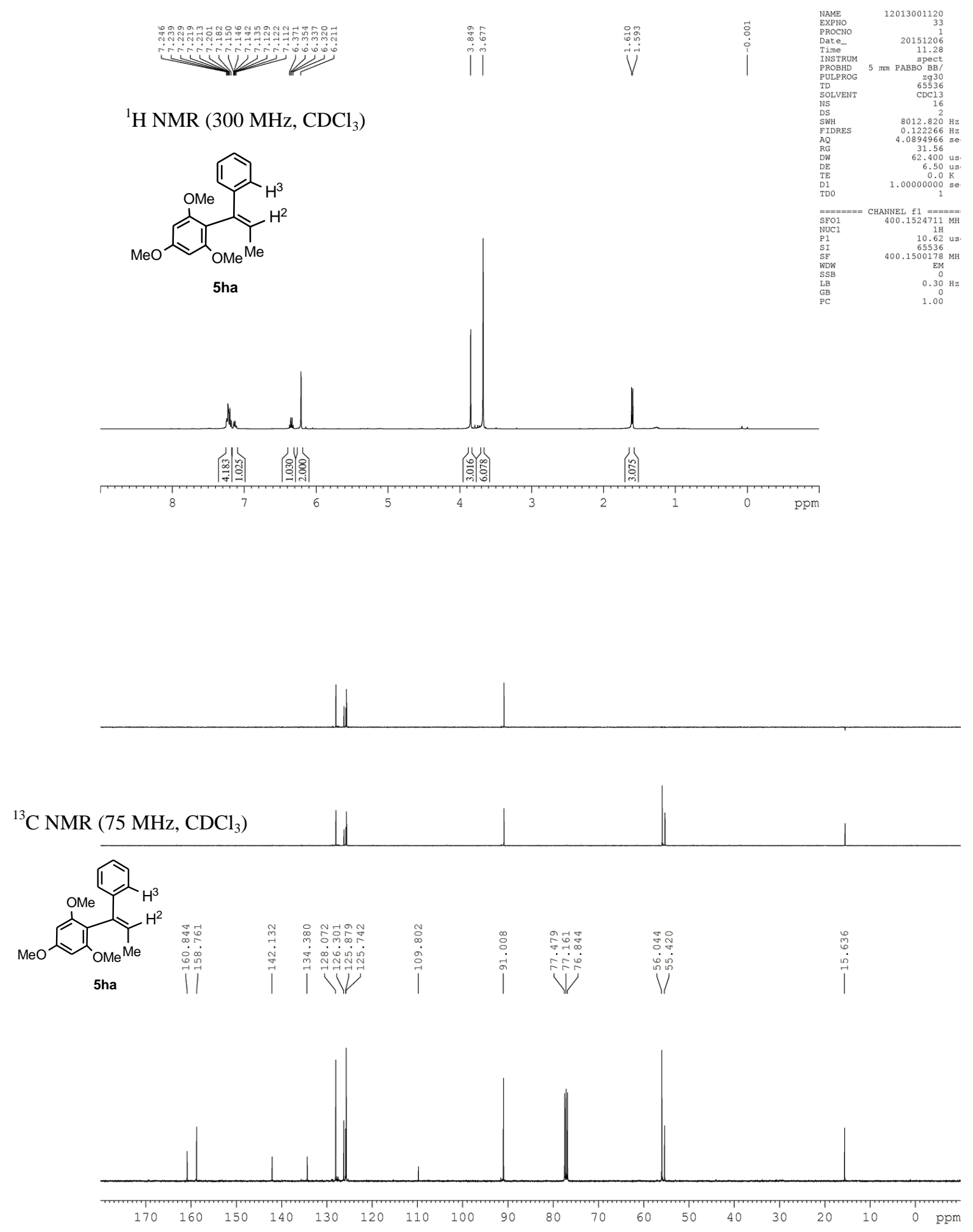


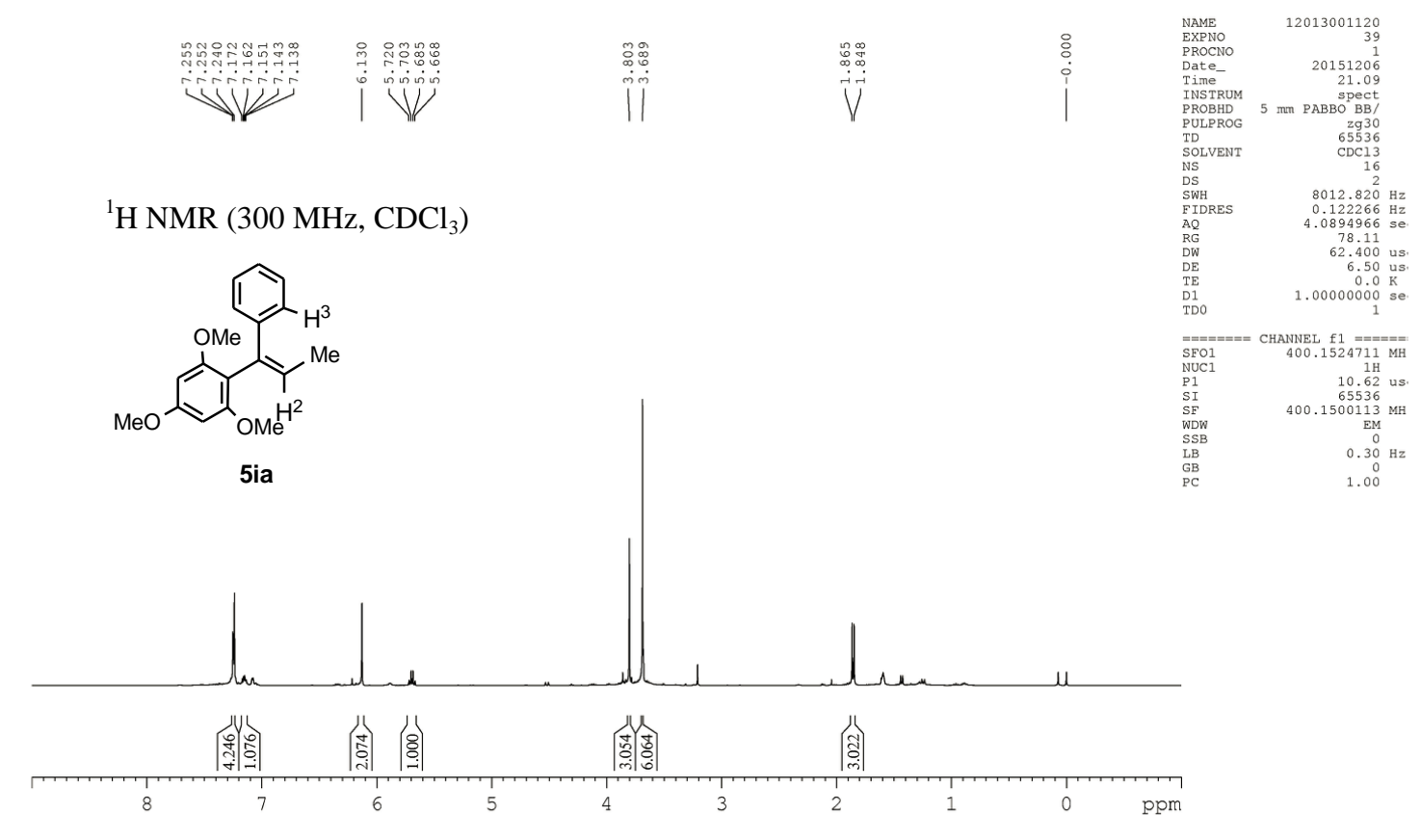

14

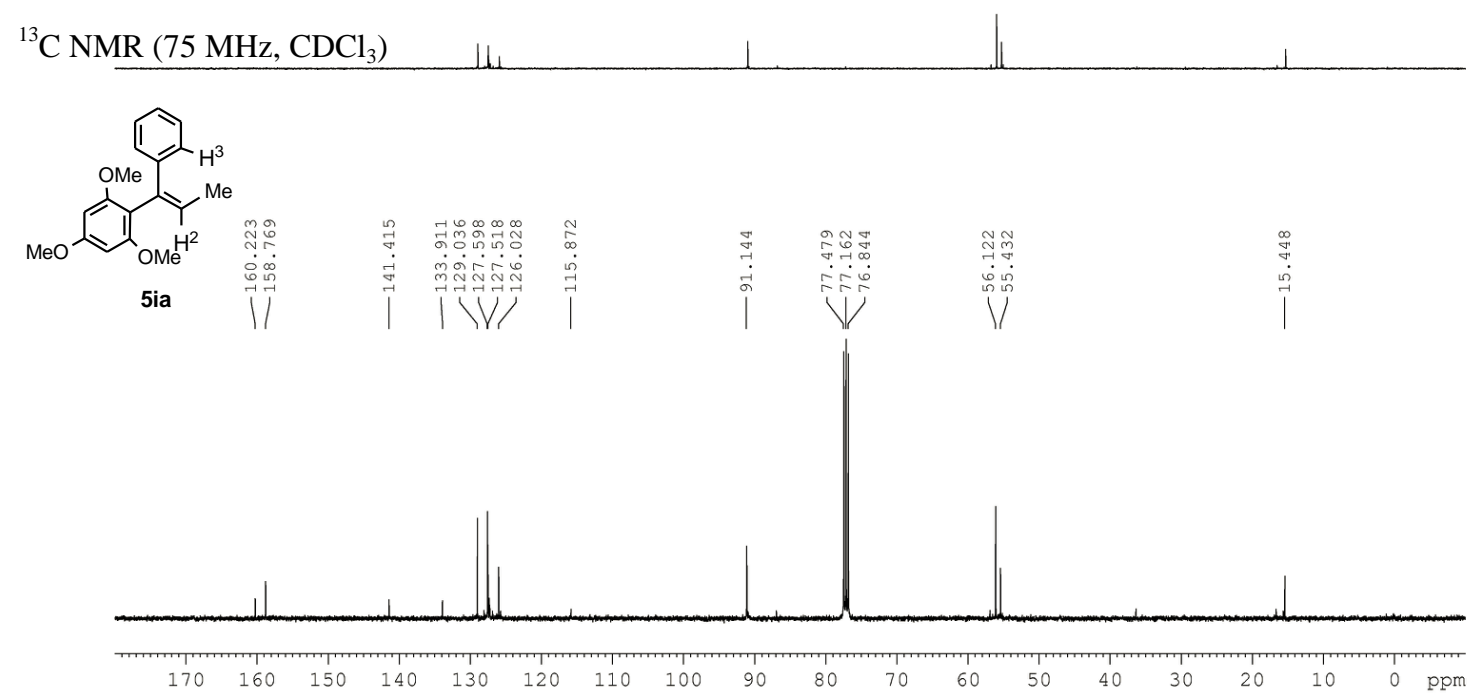




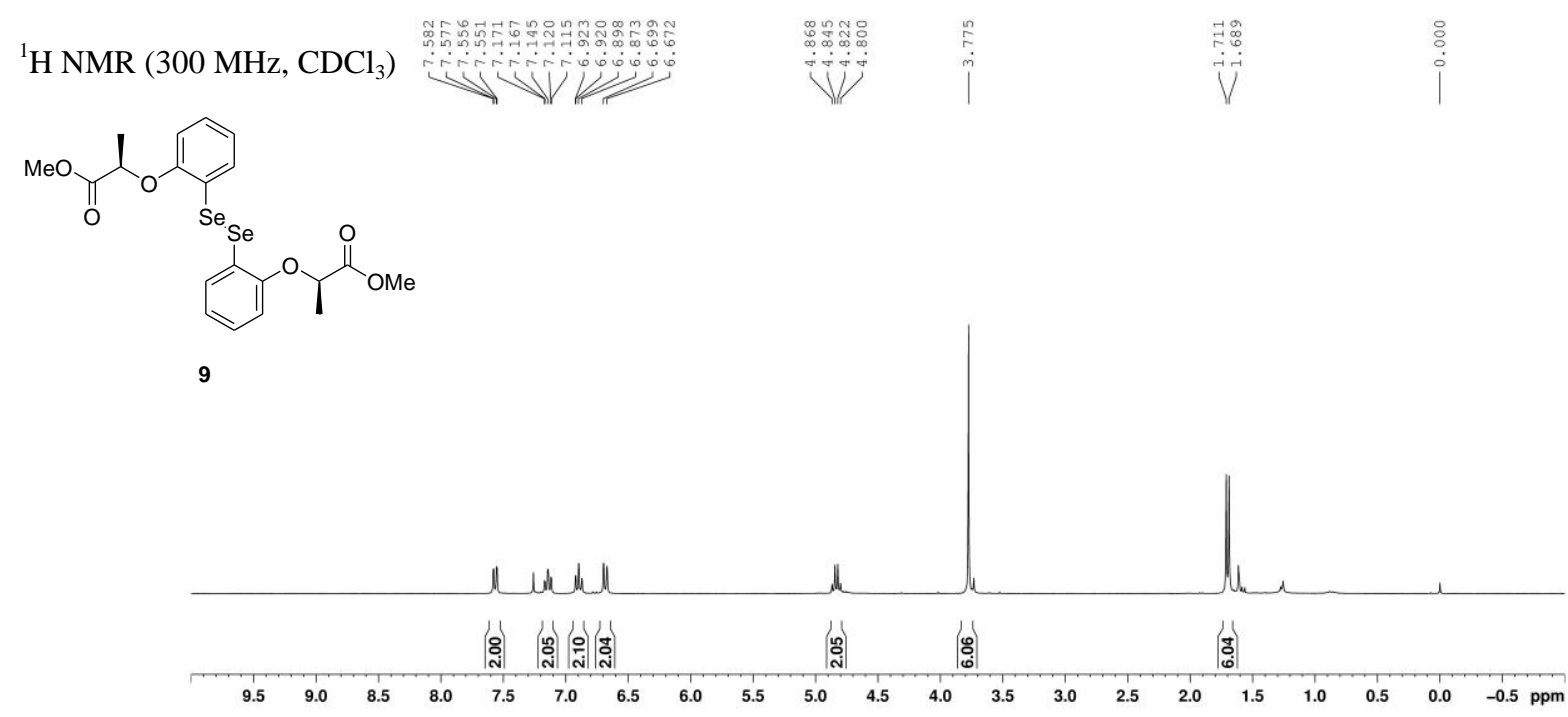

${ }^{13} \mathrm{C}$ NMR $\left(75 \mathrm{MHz}, \mathrm{CDCl}_{3}\right)$
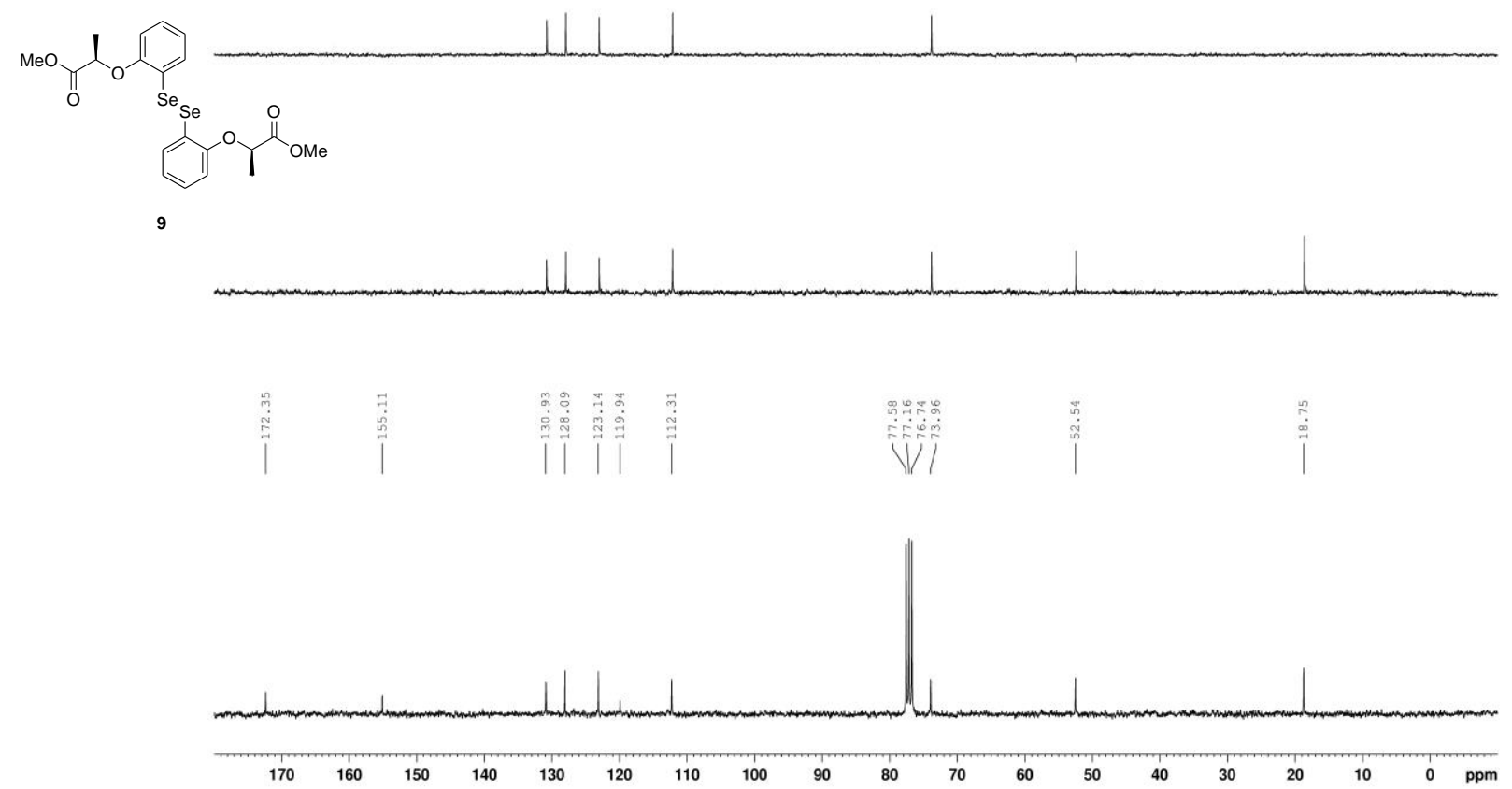
${ }^{1} \mathrm{H}$ NMR $\left(300 \mathrm{MHz}, \mathrm{CDCl}_{3}\right)$

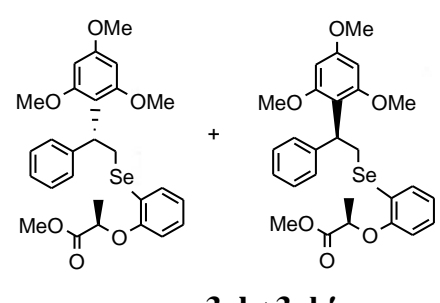

$3 \mathbf{a k}+3 \mathbf{a k}^{\prime}$

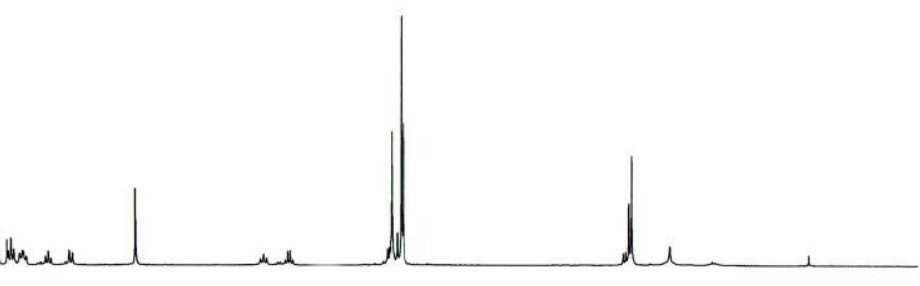

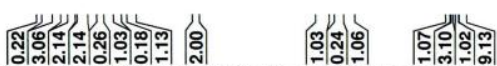

颔思

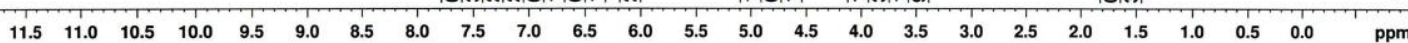

${ }^{13} \mathrm{C} \mathrm{NMR}\left(75 \mathrm{MHz}, \mathrm{CDCl}_{3}\right)$

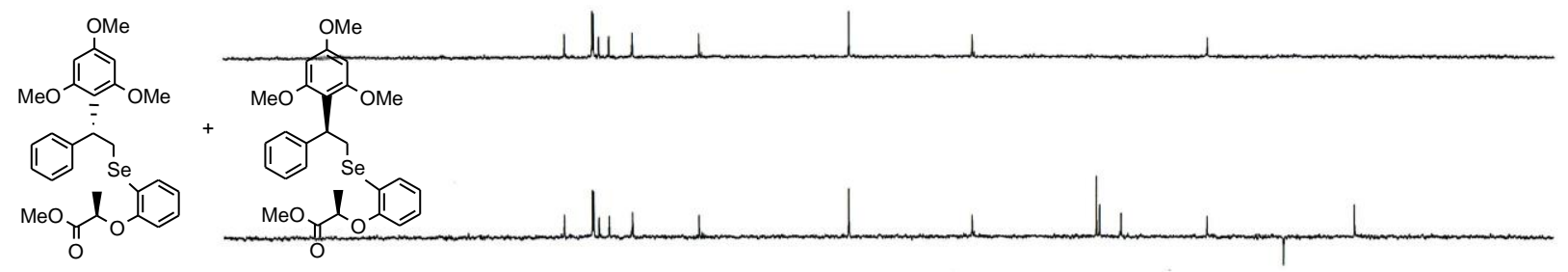

3ak+3ak'

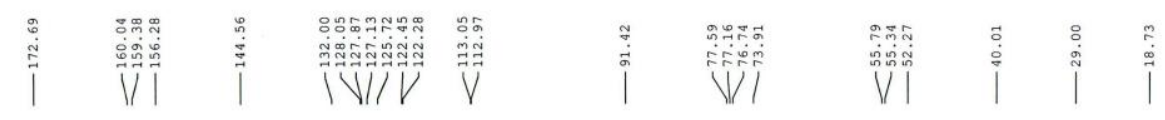

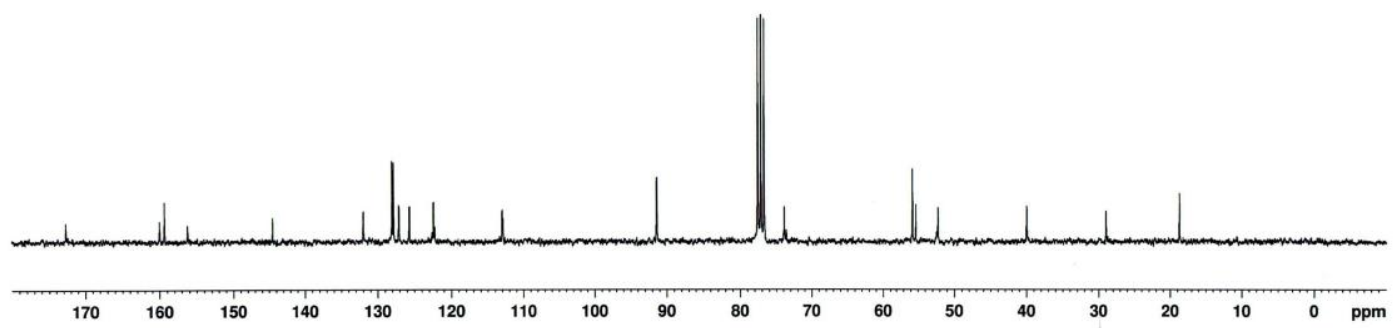




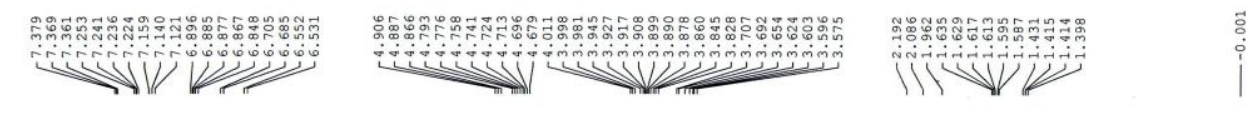

${ }^{1} \mathrm{H}$ NMR (400 MHz, $\mathrm{CDCl}_{3}$ )
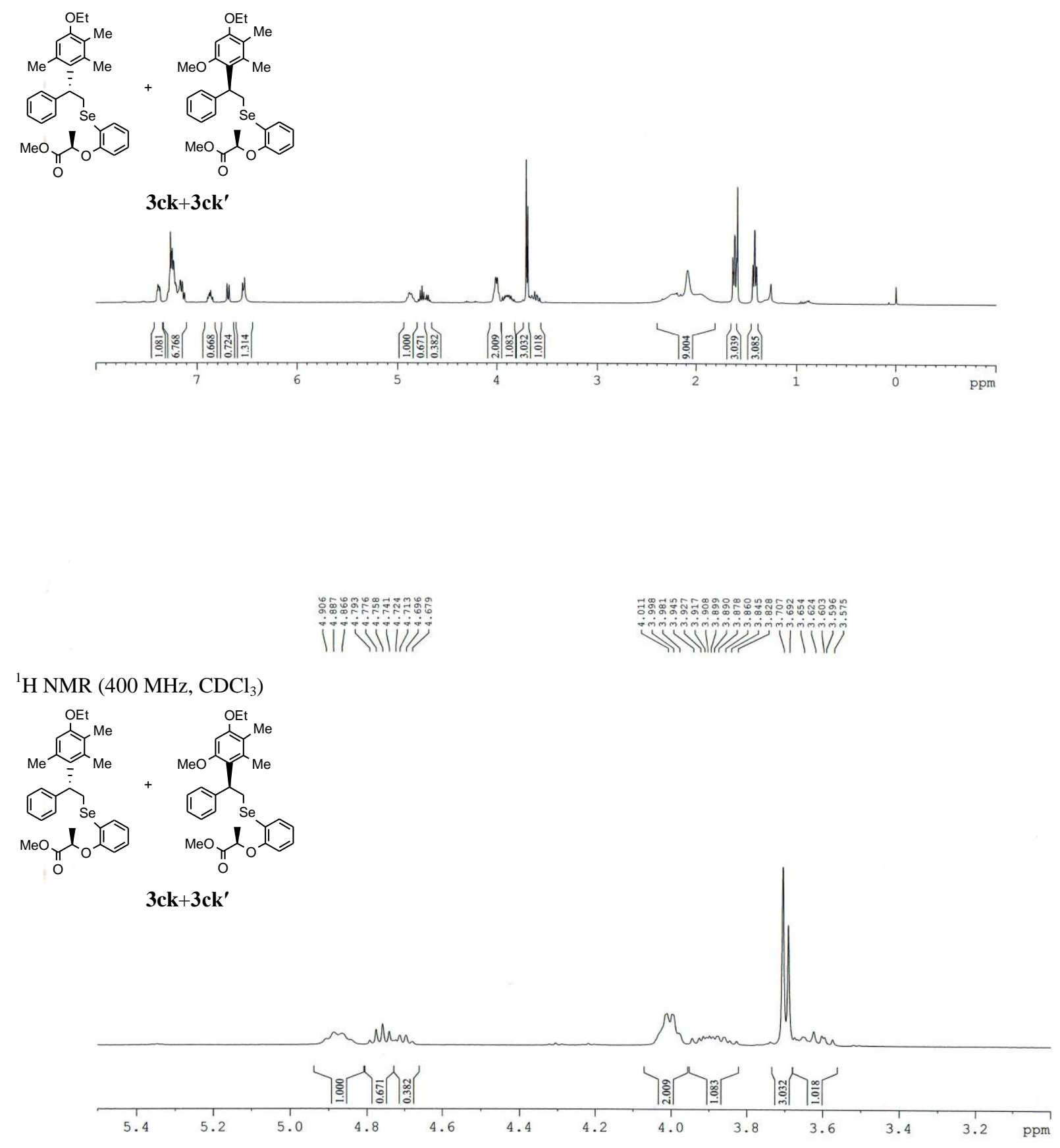
${ }^{13} \mathrm{C}$ NMR $\left(100 \mathrm{MHz}, \mathrm{CDCl}_{3}\right)$
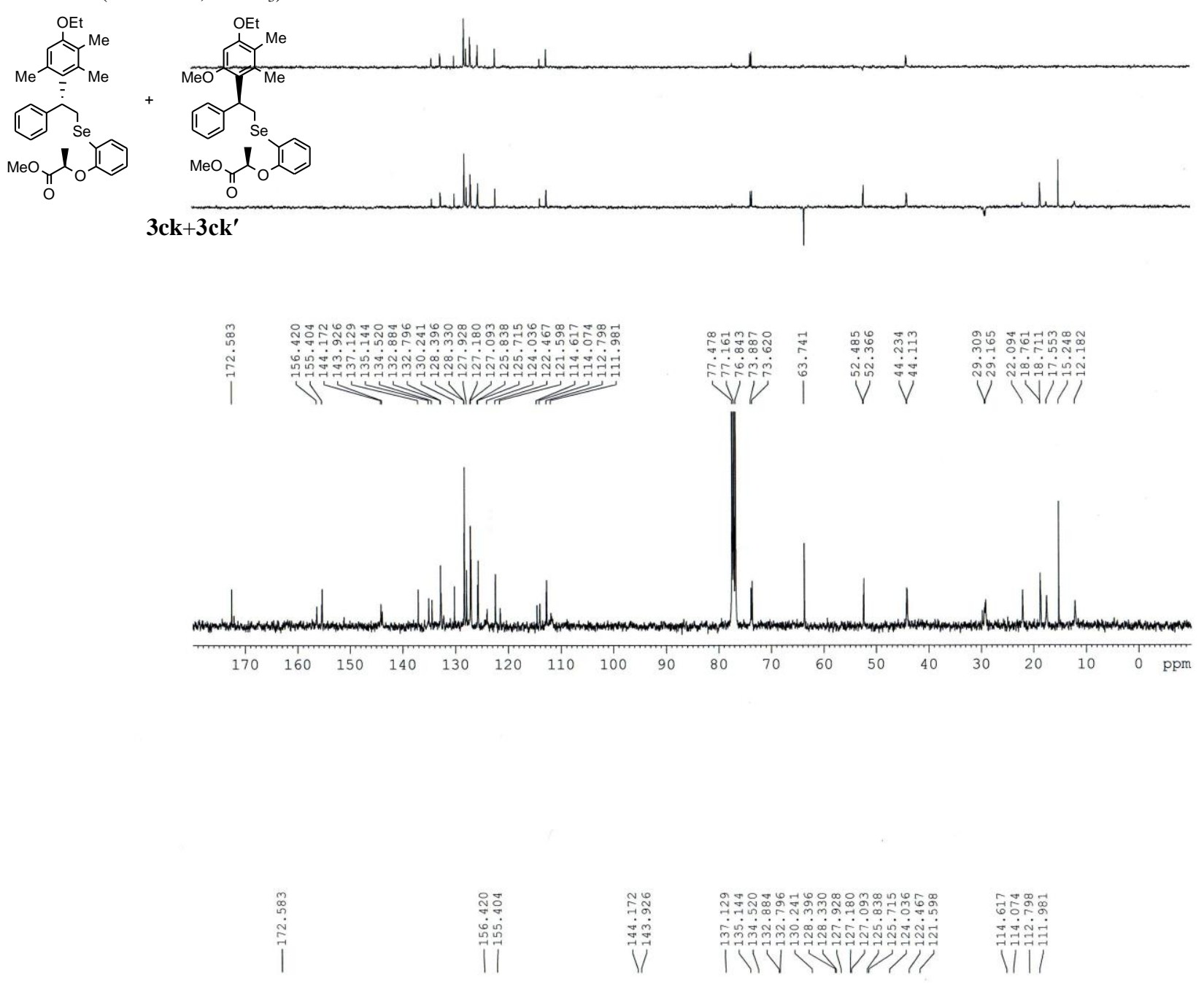

${ }^{13} \mathrm{C}$ NMR $\left(100 \mathrm{MHz}, \mathrm{CDCl}_{3}\right)$

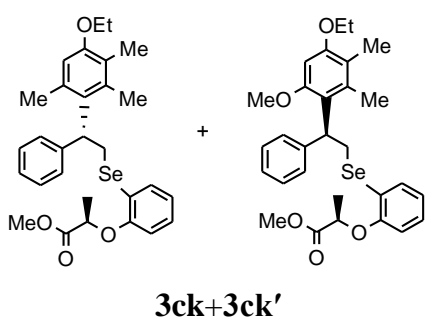

$3 \mathrm{ck}+3 \mathrm{ck}^{\prime}$

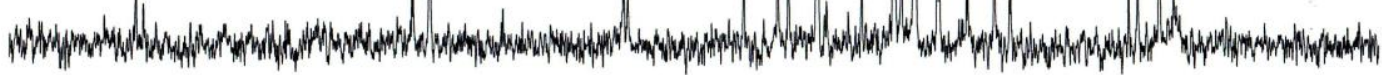

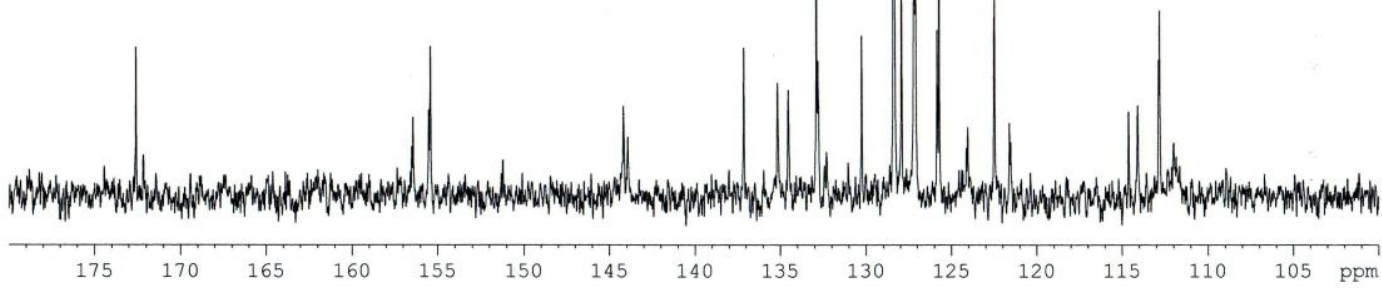


Copies of ${ }^{1} \mathrm{H}^{-1} \mathrm{H}$ COSY, HSQC, HMBC, and ROESY Spectra for compound 3ae

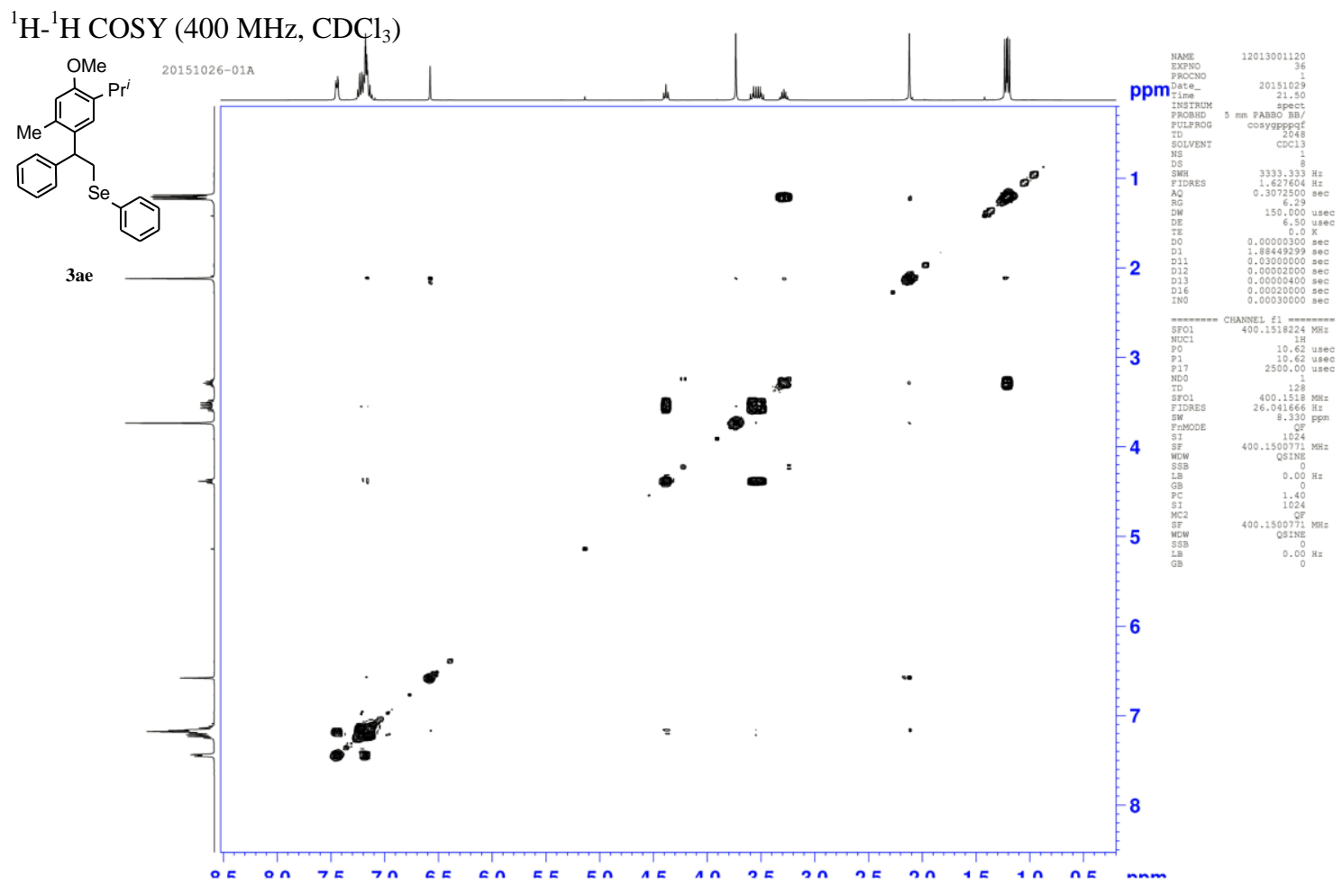

HSQC (400 MHz, $\left.\mathrm{CDCl}_{3}\right)$

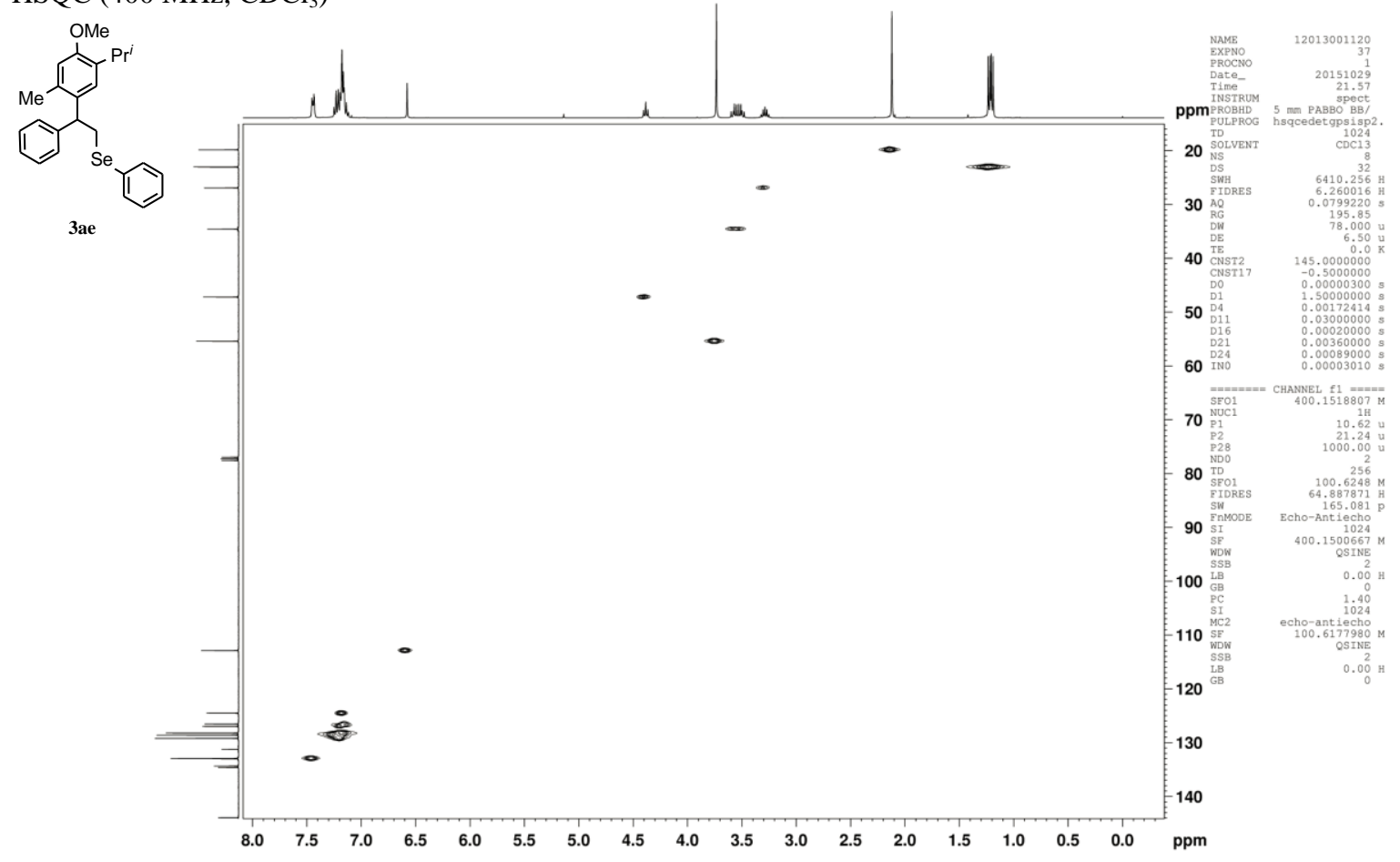

S74 

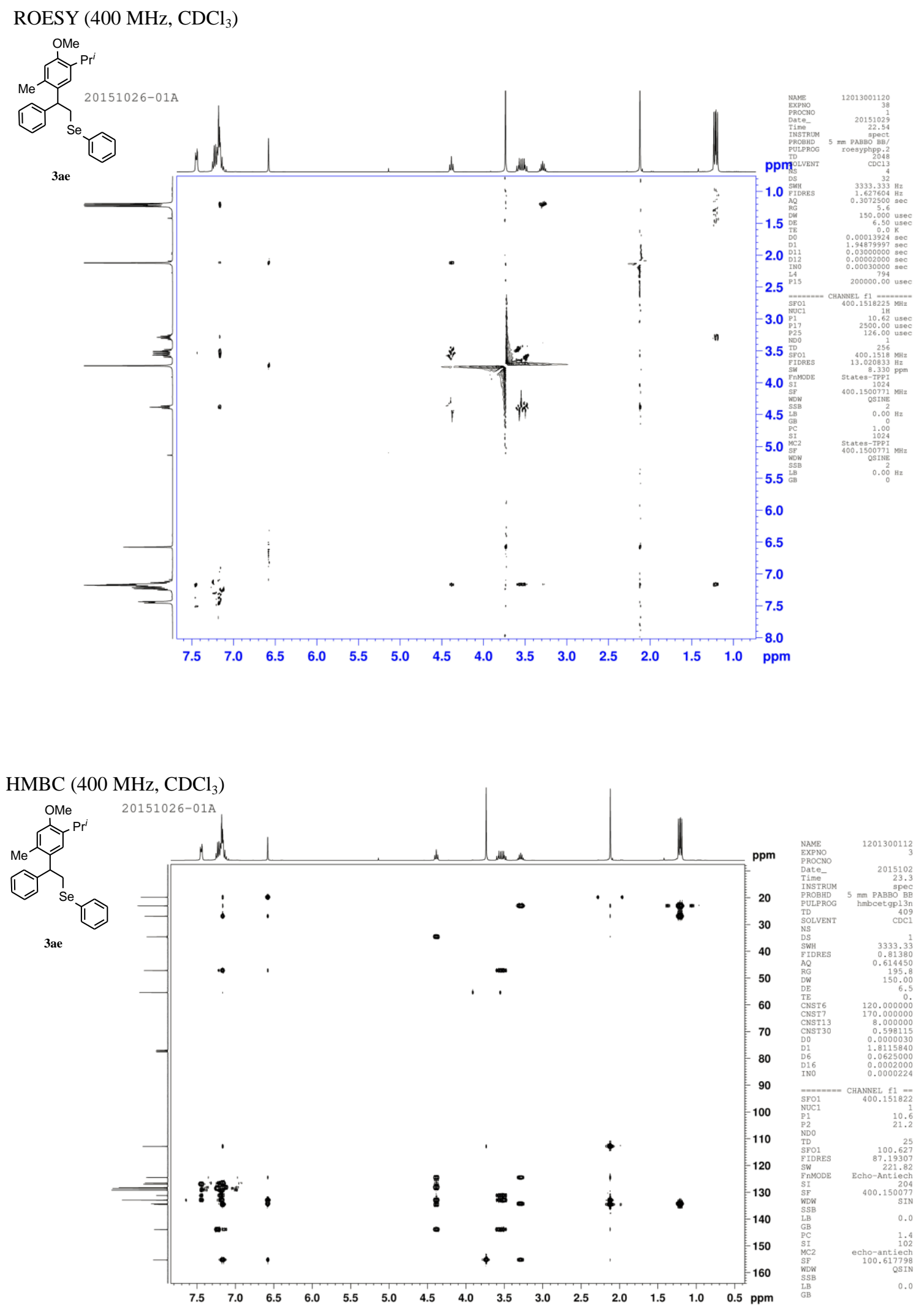


\section{Copies of ROESY Spectra for compound 5 ha, and 5ia}

${ }^{1} \mathrm{H}$ NMR (400 MHz, $\mathrm{CDCl}_{3}$ )

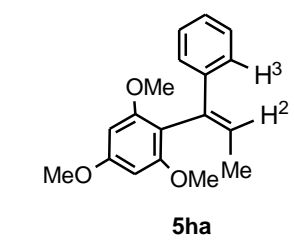

ha

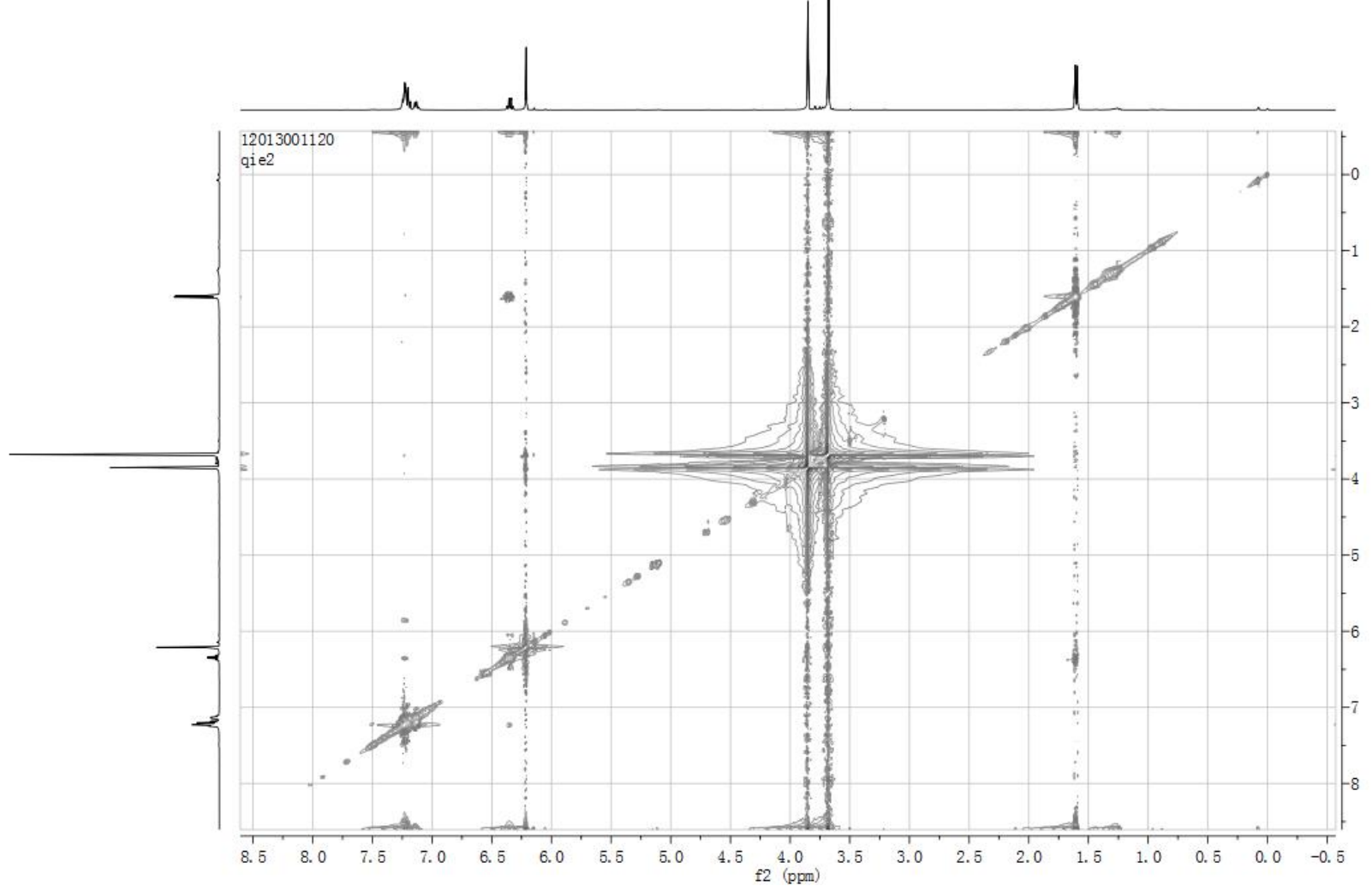




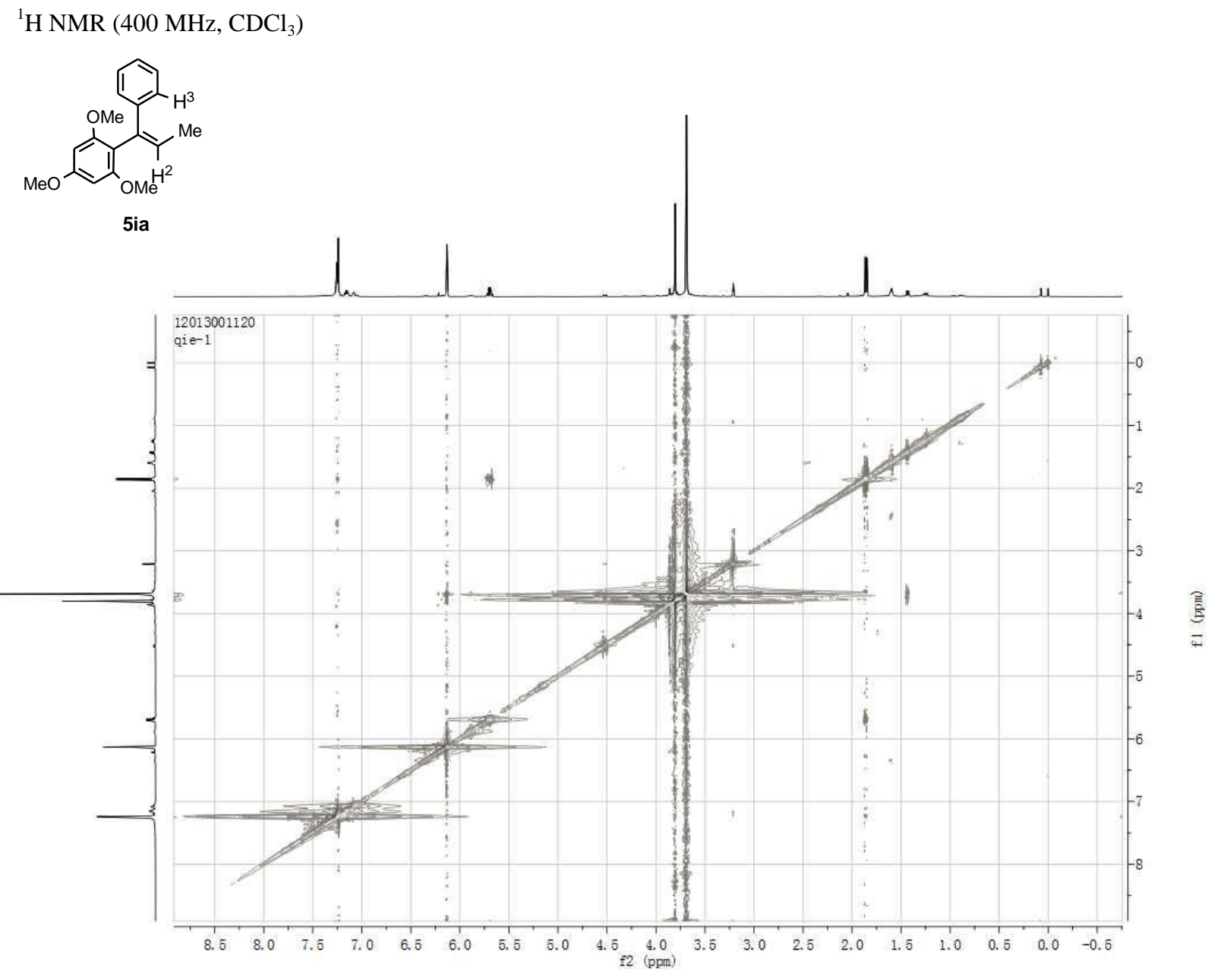

